\title{
The Miniball spectrometer
}

N. Warr ${ }^{1, a}$, J. Van de Walle ${ }^{2,3}$, M. Albers ${ }^{1, b}$, F. Ames ${ }^{4}$, B. Bastin ${ }^{5, c}$, C. Bauer ${ }^{6}$, V. Bildstein $7,8, d$, A. Blazhev ${ }^{1}$, S. Bönig ${ }^{6}$, N. Bree ${ }^{5}$, B. Bruyneel ${ }^{1, \text { e }}$, P.A. Butler ${ }^{9}$, J. Cederkäll ${ }^{2,10}$, E. Clément $^{2,11}$, T.E. Cocolios ${ }^{2,5}$, T. Davinson ${ }^{12}$, H. De Witte ${ }^{5}$, P. Delahaye ${ }^{2}$, D.D. DiJulio ${ }^{10}$, J. Diriken ${ }^{5,13}$, J. Eberth ${ }^{1}$, A. Ekström ${ }^{10}$, J. Elseviers ${ }^{5}$, S. Emhofer ${ }^{4}$, D.V. Fedorov ${ }^{14}$, V.N. Fedosseev ${ }^{2}$, S. Franchoo ${ }^{2, f}$, C. Fransen ${ }^{1}$, L.P. Gaffney ${ }^{9}$, J. Gerl ${ }^{15}$, G. Georgiev ${ }^{16}$, R. Gernhäuser ${ }^{8}$, T. Grahn ${ }^{9,17,18}$, D. Habs ${ }^{4}$, H. Hess ${ }^{1}$, A.M. Hurst ${ }^{9,19}$, M. Huyse ${ }^{5}$, O. Ivanov ${ }^{5,8}$, J. Iwanicki $^{9,20}$, D.G. Jenkins ${ }^{9,21}$, J. Jolie ${ }^{1}$, N. Kesteloot ${ }^{5,13}$, O. Kester ${ }^{4}$, U. Köster ${ }^{2}$, M. Krauth ${ }^{22}$, T. Kröll ${ }^{6,8}$, R. Krücken ${ }^{8, h}$, M. Lauer ${ }^{7}$, J. Leske ${ }^{6}$, K.P. Lieb ${ }^{23}$, R. Lutter ${ }^{4}$, L. Maier ${ }^{8}$, B.A. Marsh², D. Mücher ${ }^{1,8}$, M. Münch ${ }^{8}$, O. Niedermaier $^{7}$, J. Pakarinen ${ }^{2,9,17,18}$, M. Pantea ${ }^{6}$, G. Pascovici ${ }^{1}$, N. Patronis ${ }^{5}$, D. Pauwels ${ }^{5}$, A. Petts ${ }^{9}$, N. Pietralla ${ }^{6}$, R. Raabe $^{5}$, E. Rapisarda ${ }^{2,5}$, P. Reiter ${ }^{1}$, A. Richter ${ }^{6}$, O. Schaile ${ }^{4}$, M. Scheck ${ }^{6,9}$, H. Scheit ${ }^{6,7}$, G. Schrieder ${ }^{6}$, D. Schwalm $^{7}$, M. Seidlitz ${ }^{1}$, M. Seliverstov ${ }^{2,5,14,24, i}$, T. Sieber ${ }^{2}$, H. Simon ${ }^{6}$, K.-H. Speidel ${ }^{25}$, C. Stahl ${ }^{6}$, I. Stefanescu ${ }^{5,26}$, P.G. Thirolf $^{4}$, H.-G. Thomas ${ }^{1}$, M. Thürauf ${ }^{6}$, P. Van Duppen ${ }^{5}$, D. Voulot ${ }^{27}$, R. Wadsworth ${ }^{21}$, G. Walter ${ }^{22,28}$, D. Weißhaar ${ }^{1, j}$, F. Wenander ${ }^{27}$, A. Wiens ${ }^{1, k}$, K. Wimmer ${ }^{8,1}$, B.H. Wolf ${ }^{4}$, P.J. Woods ${ }^{12}$, K. Wrzosek-Lipska ${ }^{5,20}$, and K.O. Zell ${ }^{1}$

1 Institut für Kernphysik, Universität zu Köln, Zülpicher Straße 77, D-50937 Köln, Germany

2 PH Department, ISOLDE; CERN, CH-1211, Geneva, Switzerland

3 Kernfysisch Versneller Instituut, University of Groningen, Zernikelaan 25, 9747 AA Groningen, The Netherlands

4 Sektion Physik, Ludwig-Maximilians-Universität, München, D-85748 Garching, Germany

5 Instituut voor Kern- en Stralingsfysica, KU Leuven, B-3001 Leuven, Belgium

6 Institut für Kernphysik, Technische Universität Darmstadt, D-64289 Darmstadt, Germany

7 Max-Planck-Insitut für Kernphysik, Heidelberg, D-69117, Germany

8 Physik Department E12, Technische Universität München, D-85748 Garching, Germany

9 Oliver Lodge Laboratory, University of Liverpool, L69 7ZE, England, UK

10 Fysiska Institutionen, Lunds Universitet, Box 118, 22100 Lund, Sweden

11 Grand Accélérateur National d'Ions Lourds (GANIL), F-14021 Caen CEDEX, France

12 Department of Physics and Astronomy, University of Edinburgh, EH9 3JZ, Scotland, UK

13 Studiecentrum voor Kernenergie/Centre d'Etude de l'nergie Nuclaire (SCK CEN), B-2400 Mol, Belgium

14 Department of High Energy Physics, Petersburg Nuclear Physics Institute, Gatchina, Russia

15 Gesellschaft für Schwerionenforschung, Planckstraße 1, D-64291 Darmstadt, Germany

16 CSNSM, CNRS/IN2P3, Université Paris-Sud 11, UMR8609, F-91405 ORSAY-Campus, France

17 Department of Physics, University of Jyväskylä, FI-40014, Jyväskylä, Finland

18 Helsinki Institute of Physics, P.O. Box 64, FI-00014, Helsinki, Finland

19 Lawrence Berkeley National Laboratory, MailStop 88R0192, 1 Cyclotron Road, Berkeley, CA 94720, USA

20 Heavy Ion Laboratory, Warsaw University, Pasteura 5A, 02-093 Warsaw, Poland

21 Nuclear Physics Group, Department of Physics, University of York, YO10 5DD, England, UK

22 Institut de Recherches Subatomiques, F-67037 Strasbourg CEDEX 2, France

23 II. Physikalisches Institut, Universität Göttingen, D-37073, Göttingen, Germany

24 School of Engineering and Science, University of West Scotland, PA1 2BE Paisley, UK

25 Helmholtz-Institut für Strahlen-und Kernphysik, Universität Bonn, Nußallee 14-16, D-53115 Bonn, Germany

26 Department of Chemistry and Biochemistry, University of Maryland, College Park, Maryland 20742, USA

27 AB Department, ISOLDE; CERN, CH-1211, Geneva, Switzerland

28 Université Louis Pasteur, 4 rue Blaise Pascal, CS 90032, F-67081 Strasbourg CEDEX, France

Received: 23 November 2012 / Revised: 25 January 2013

Published online: 26 March 2013

(C) The Author(s) 2013. This article is published with open access at Springerlink.com

Communicated by J. Äystö 


\begin{abstract}
The Miniball germanium detector array has been operational at the REX (Radioactive ion beam EXperiment) post accelerator at the Isotope Separator On-Line facility ISOLDE at CERN since 2001. During the last decade, a series of successful Coulomb excitation and transfer reaction studies have been performed with this array, utilizing the unique and high-quality radioactive ion beams which are available at ISOLDE. In this article, an overview is given of the technical details of the full Miniball setup, including a description of the $\gamma$-ray and particle detectors, beam monitoring devices and methods to deal with beam contamination. The specific timing properties of the REX-ISOLDE facility are highlighted to indicate the sensitivity that can be achieved with the full Miniball setup. The article is finalized with a summary of some physics highlights at REX-ISOLDE and the utilization of the Miniball germanium detectors at other facilities.
\end{abstract}

\section{Introduction}

The high-resolution Miniball germanium detector array has been operational at REX-ISOLDE at CERN for over 10 years. This array consists of 24 six-fold segmented, tapered, encapsulated high-purity germanium crystals and was specially designed for low multiplicity experiments with low-intensity radioactive ion beams (RIB). The Miniball array has been used in numerous Coulomb-excitation and transfer-reaction experiments with exotic RIBs with energies up to $\approx 3 \mathrm{MeV} / A$, produced at the ISOLDE facility. In this review article, the experimental setup is described in detail. The germanium array and its infrastructure is described in sect. 2 and the particle detectors are described in sect. 3 . In sect. 4 , the relevant time structure of the (REX-)ISOLDE facility is described, since it has a profound impact on the data taking and sensitivity achievable at the Miniball setup. The beam monitoring devices and the methods to deal with severe beam contamination of the post-accelerated RIBs are discussed in sects. 5 and 6 , respectively. Section 7 describes the methods used to Doppler-correct the $\gamma$-ray spectra. Section 8 provides some of the physics highlights from the past 10 years, obtained at REX-ISOLDE. Section 9 describes the use of the Miniball germanium detectors at other laboratories (GSI and LISOL). Additional information on the REX-ISOLDE post-accelerator can be found in [1], first reports on the Miniball array in [2] and a recent review focused on the REX-ISOLDE physics program in $[3]$.

\section{The germanium detector array Miniball}

\subsection{Requirements imposed on detector design by radioactive beam experiments}

Since the 1980s large Ge detector arrays such as EUROBALL [4] and GAMMASPHERE [5] have provided a wealth of data near stability and on the proton-rich side of the nuclear chart. Neutron-rich nuclei, however, are not generally accessible to the heavy-ion reactions that these arrays were designed for. The advent of rare isotope beam facilities makes it possible to produce, separate and postaccelerate beams that cannot easily be provided by other methods, opening up new parts of the nuclear chart for exploration.

Due to the generally lower energy and intensity as compared to stable beam facilities, the obvious reactions to perform with post-accelerated radioactive beams are Coulomb excitation and particle transfer reactions. However, in such experiments, the isotopes of interest are the projectiles and not the target nuclei with the result that the particle emitting the $\gamma$-rays of interest may be moving fast, leading to large Doppler shifts. Given the finite solid angle subtended by the detectors, this gives rise to considerable Doppler broadening. This can be corrected for, when the detection system has high granularity, making it possible to determine the angle between the recoiling nucleus and the $\gamma$-ray accurately. The way this granularity was obtained for EUROBALL and GAMMASPHERE was to use a large number of detectors at a significant distance to the target, so that each subtends a relatively small angular range. This is, in any case, necessary for high-spin studies, where the multiplicities are high.

a e-mail: warr@ikp.uni-koeln.de

b Present address: ANL, 9700 S Cass Avenue, Argonne, IL 60439, USA.

c Present address: GANIL, CEA/DSM-CNRS/IN2P3, Boulevard H. Becquerel, F-14076, Caen, France.

d Present address: Department of Physics, University of Guelph, Guelph ON, N1G 2W1, Canada.

e Present address: CEA Saclay, F-91191 Gif-sur-Yvette, France Cedex.

f Present address: CSNSM, CNRS/IN2P3, Université Paris-Sud 11, UMR8609, F-91405 ORSAY-Campus, France.

g Present address: SUE SIA "Radon", The 7-th Rostovsky Lane 2/14, Moscow, 119121, Russia.

h Present address: TRIUMF, 4004 Wesbrook Mall, Vancouver, BC, V6T 2A3, Canada.

i Present address: Scottish Universities Physics Alliance, University of Glasgow, Glasgow G12 8QQ, Scotland, UK.

j Present address: NSCL, MSU, East Lansing, MI 48824, USA.

k Present address: LBNL, 1 Cyclotron Road, Berkeley, CA 94720, USA.

1 Present address: Department of Physics, Central Michigan University, Mount Pleasant, MI 48859, USA. 


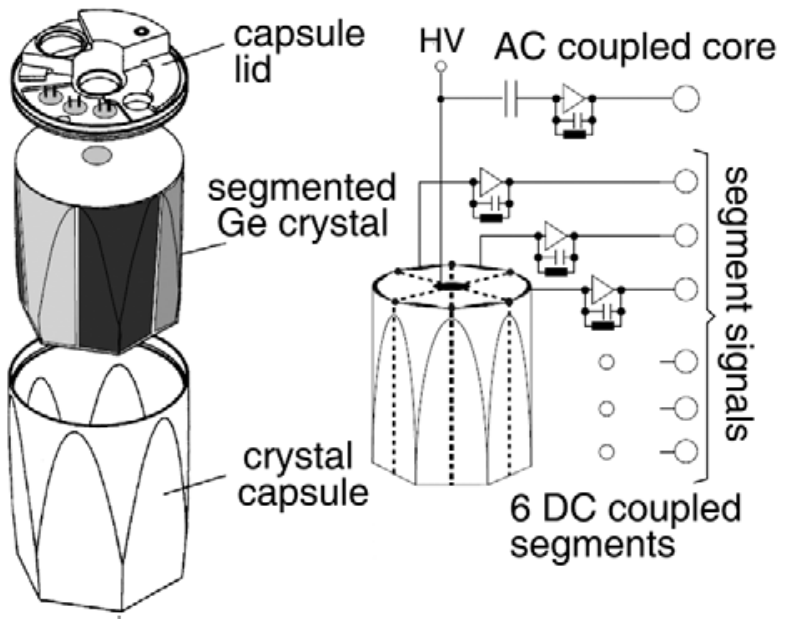

Fig. 1. Layout of one segmented germanium crystal. Left-hand side: an exploded view of the capsule assembly. Right-hand side: a schematic view of the readout circuitry.

For work with rare-isotope beams, on the other hand, the multiplicities are low (often only a few states are excited) and the yields of such beams are usually much lower than for conventional experiments, so efficiency is paramount. In the mid 1990s it was realized [6] that granularity could be achieved in another way, namely by the segmentation of the charge-collection electrodes of the Ge detectors and the use of pulse-shape analysis to determine the position of the first interaction of the $\gamma$-ray within the Ge crystal, giving a spatial resolution significantly finer than the dimensions of the crystal [7].

Simulations showed that this novel technique could be used to build a spectrometer with a close and compact geometry, consisting of much fewer detectors than either EUROBALL or GAMMASPHERE, but yet obtaining a higher granularity than that of these spectrometers through the use of pulse-shape analysis with segmented detectors $[8,9]$. The Miniball array, consisting of 24 sixfold segmented germanium crystals, was built to pioneer this technique [2].

Another consequence of the low multiplicity is that the advantage of Compton suppression in cleaning up the spectra is outweighed by the loss in efficiency, that the addition of shields entails. For this reason, Miniball was constructed without Compton-suppression shields, but with the option of adding such shields later when higher beam energies become available at REX-ISOLDE, allowing experiments with higher $\gamma$-ray multiplicities.

\subsection{Design of the Miniball cluster detectors}

The basic constituents of the Miniball detector array are the 24 high-purity germanium crystals, which have the same quasi-cylindrical shape with a hexagonally tapered front end, as those used for the EUROBALL cluster detector [10], with a diameter of $70 \mathrm{~mm}$ (before shaping) and a height of $78 \mathrm{~mm}$. A drawing of one Miniball crystal is shown schematically in fig. 1 .

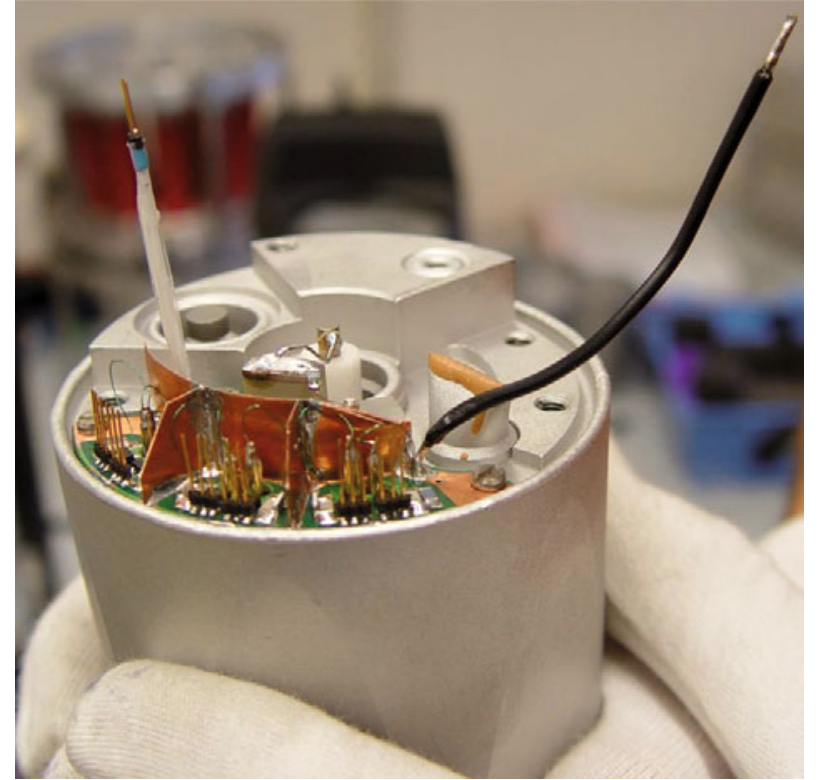

Fig. 2. (Color online) A Miniball capsule with the preamplifier board mounted. The six FETs and their feedback circuits for the segments are at the front, with the FET for the core and its feedback circuit hidden by the copper shielding. The coupling capacitor can just be seen above this copper shielding with the $\mathrm{HV}$ wire going to the core electrode.

The main difference compared to the EUROBALL crystals is the additional six-fold electrical segmentation of the outer electrode. Each crystal yields six segment signals and a sum energy obtained from the core electrode. High voltage is applied to the central core contact, which is AC-coupled to its preamplifier. The six segments are DCcoupled to their respective preamplifiers. The cold parts of the preamplifiers are illustrated schematically in fig. 1. The cold parts of all seven preamplifiers and the coupling capacitor are mounted on a board on top of the capsule lid, which is cooled together with the germanium crystal. As can be seen from fig. 2, the seven field effect transistors (FET) with their feedback circuits and the coupling capacitor for the core occupy a space roughly equivalent to that occupied by the cold part of a single EUROBALL preamplifier. The sources of all seven FETs are connected to a common ground in the cold part of the detector, while the feedback and drain of each FET are fed separately into the "warm" (i.e. not cryogenically cooled) part, where they are connected to the warm parts of the preamplifiers, which are shown in fig. 3. The benefit of the six-fold segmentation in the context of the Doppler correction procedure will be shown in sect. 7 .

The germanium crystals are encapsulated in a permanently sealed aluminum can ("crystal capsule") using the technique pioneered by EUROBALL [11], which makes it possible to access the cold electronics without needing a clean room. The success of this technique led to its adoption by Miniball, as is illustrated in fig. 1. The high density of the electronics together with the need for AC coupling of the core electrode increases the chances of damaging an FET in the event of problems with either the vacuum of the cryostat (e.g., caused by a poor cooling cycle, lead- 


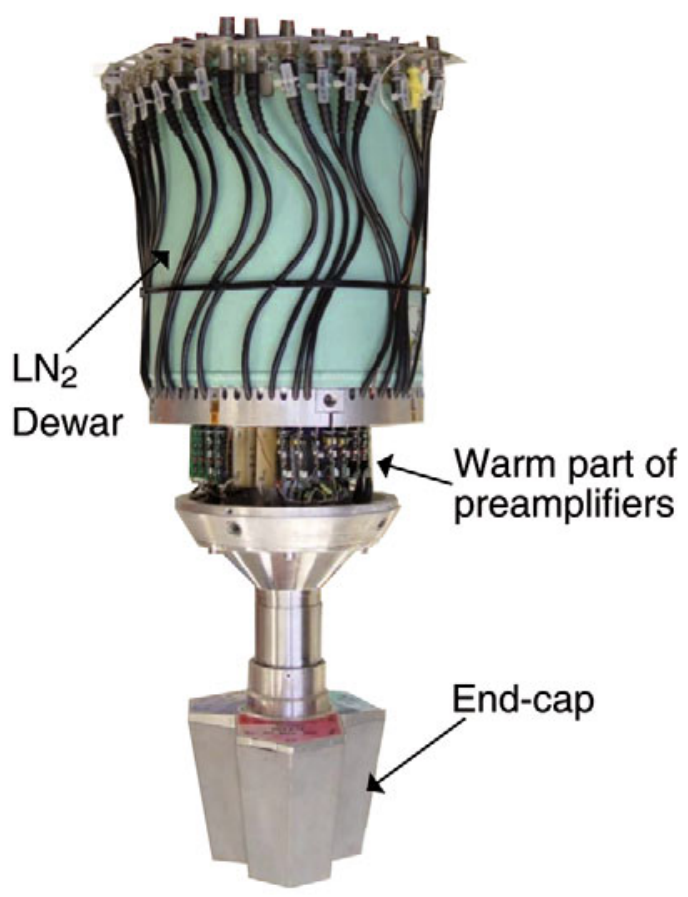

Fig. 3. (Color online) Picture of a Miniball cluster detector consisting of three encapsulated HPGe crystals. The preamplifier housing has been removed so the warm parts of the preamplifiers can be seen.

ing to a partial warm up which causes out-gassing) or from HV instabilities. The encapsulated design ensures that such damage can be repaired in situ at ISOLDE and has been crucial for the long-term reliability of Miniball.

The Miniball clusters have cryostats, which are designed to house either three or four of the encapsulated six-fold segmented Miniball detectors, with just a change in the end-cap to switch between the triple and quad versions. At present only the triples are in use. For each of a maximum of four capsules, there are vacuum feedthroughs between the cold and warm part for the HV, for the seven feedback signals and for the seven drain signals. In addition, there is a feedthrough for a single PT100 temperature sensor placed next to one of the capsules, which is used by the liquid nitrogen $\left(\mathrm{LN}_{2}\right)$ filling system to monitor the temperature (see sect. 2.8). The germanium crystals are mounted on a cold finger which is cooled by $\mathrm{LN}_{2}$ stored in the Dewar behind. High vacuum (typically $10^{-6} \mathrm{mbar}$ ) is maintained within the thin aluminum end-cap to ensure the thermal insulation of the cold crystals from the outside.

The warm part of the cryostat was designed to have space for the electronics for four capsules, each with a high-voltage filter and the warm part of seven preamplifiers (see sect. 2.3) mounted on a motherboard. In the Miniball triple clusters, electronics for only three capsules is present, leaving an empty space where the electronics for a fourth capsule would go. There is a second PT100 sensor on the cold finger, which can be used to check the temperature close to the Dewar, and there is also a heating ele- ment, which can be used for annealing and/or baking out while pumping. This full configuration is shown in fig. 3 . Each preamplifier has two identical outputs, but in the original design only one of these was connected via BNC cables to a patch panel, since the use of a digital system (see sect. 2.5) means that the energy and time branches are separated only after digitization. However, the use of Miniball clusters in hybrid systems with dual digital and analog branches at other facilities has shown that, for the core signals at least, both outputs are needed. A recent upgrade has cabled up the second output for each of the three core signals, making it possible to have separate time and energy branches for use with data acquisitions that require this.

\subsection{Preamplifiers}

The requirements of the front-end electronics developed for the Miniball array are imposed firstly by the use of 6-fold segmented HPGe detectors and secondly by the early decision to use a data acquisition system based on commercial fast digitizers [12] in order to implement the pulse-shape analysis technique to extract not only the energy and time information, but also to determine the twodimensional position of the $\gamma$-ray interaction in the Ge crystal.

The use of the pulse-shape analysis technique for the signals collected from segmented detectors raises an additional issue for the preamplifier specification, namely a fast rise time (faster than the fastest collection time of electrons and holes in a detector) and, moreover, a very clean transfer function in the time domain, which has to be preserved in cryostats equipped with multiple segmented detectors and real wiring. Furthermore the crosstalk between channels has to be as small as possible (by design it is in the order of magnitude of $\sim 10^{-3}$ or less). The specifications achieved by the Miniball preamplifiers are detailed in table 1.

A very large dynamic range at high counting rates is mandatory to detect $\gamma$-rays alone in the range up to 10-20 MeV. Special care has to be taken in the design of the front-end analog electronics to avoid saturation due to pile-up effects of those signals above $30 \mathrm{kcps}$.

The structure of the front-end electronics consists of the following stages: a charge-sensitive loop including a part cooled cryogenically, a pole-zero cancellation with integrated fast reset circuitry, and an output buffer with two unipolar outputs in the case of 6-fold segmented detectors.

The input stage collects charge due to electrons at the central electrode or due to holes at each of the 6 segment electrodes. The connection to the central electrode is AC $(1 \mathrm{nF})$ due to a $4-5 \mathrm{kV}$ bias voltage applied to this electrode and DC to the all segments. The charge-sensitive cooled part comprises an input stage with a very low noise jFET-type FET and a passive feedback network. These components are placed in the cryostat near the detector contacts, to reduce the noise and the microphonic effects. The input jFET has optimal performance with an equivalent noise voltage of $\sim 0.6 \mathrm{nV} / \sqrt{\mathrm{Hz}}$ at a temperature in 
Table 1. Technical specification of the Miniball front-end electronics.

\begin{tabular}{|l|l|l|}
\hline Property & Value & Tolerance \\
\hline Conversion gain for core/segments & $\sim 175 \mathrm{mV} / \mathrm{MeV}($ optionally $350 \mathrm{mV})$ & $\pm 10 \mathrm{mV}$ \\
\hline Noise & $<0.6 \mathrm{keV}$ FWHM $\left(C_{d}=0 \mathrm{pF} @ 150 \mathrm{~K}\right)$ & \\
\hline Noise slope & $+8 \mathrm{eV} / \mathrm{pF}$ & $\pm 2 \mathrm{eV}$ \\
\hline Rise time & $\sim 13 \mathrm{~ns}(0 \mathrm{pF})$ & $\pm 2 \mathrm{~ns}$ \\
\hline Rise-time slope & $\sim 0.3 \mathrm{~ns} / \mathrm{pF}$ & $\pm 5 \mu \mathrm{s}$ \\
\hline Decay time & $50 \mu \mathrm{s}$ & \\
\hline Integral non-linearity & $<0.025 \%($ dynamic range $\sim 8.75 \mathrm{~V})$ & \\
\hline $\begin{array}{l}\text { Output polarity selectable: } \\
\text { - dual single: positive or negative }\end{array}$ & Single ended, $Z_{0}=50 \Omega$ & $\pm 0.5 \mathrm{~V}$ \\
\hline Power supply & $\pm 12.0 \mathrm{~V}$ & \\
\hline Power consumption jFET & $50 \mathrm{~mW} \mathrm{IF1320}$ & \\
\hline Power consumption at low counting rates & $<350 \mathrm{~mW}$ warm part $+\mathrm{jFET}$ power consumption (cold part) & \\
\hline
\end{tabular}

the range $\approx 150 \mathrm{~K}$. The actual temperature of the FET cannot easily be determined, but the heat produced by the FET itself raises its temperature above that of the cryogenically cooled capsule on which it is mounted. The feedback network time constant of the first stage is $1 \mathrm{~ms}$ (1 G $\Omega$ and $1 \mathrm{pF}$, respectively) to optimize both, noise and bandwidth in the first stage.

The Transimpedance Amplifier (TA) designed with discrete components is operated at room temperature outside the cryostat. A "cascode" structure was adopted for the TA stage. The TA is optimized for a wide unipolar output range of $\sim 10 \mathrm{~V}$ positive or negative swings. As the closed-loop gain is $\sim 53 \mathrm{mV} / \mathrm{MeV}$ and the output voltage range of the TA stage is about $\sim 10 \mathrm{~V}$, we obtain an energy range of $\sim 180 \mathrm{MeV}$ with an intrinsic noise of $\sim 600 \mathrm{eV}$ at $150 \mathrm{~K}$ and with a slope of $8 \mathrm{eV} / \mathrm{pF}$ (the total detector bulk capacitance being $\sim 39 \mathrm{pF}$ ).

To get a fast rise time at lowest possible noise, several jFET structures have been tested and the IF1320 (InterFET) with a working point at $U_{d} \sim 5 \mathrm{~V}, I_{d} \sim 10 \mathrm{~mA}$ $(\sim 50 \mathrm{~mW})$ was selected for the 6 -fold segmented detector. The printed circuit boards for the cold part were designed for this type of jFET and, moreover, the adjacent channels are shielded to minimize the electromagnetic interference and the crosstalk between channels.

The second stage of the preamplifier section is a passive pole-zero cancellation $(P / Z)$ network and a buffer stage as impedance matcher. The $P / Z$ stage has the purpose of reducing the decay time of the first charge-sensitive stage (of $1000 \mu \mathrm{s}$ ) down to $50 \mu \mathrm{s}$. Therefore, the baseline restoration is faster and the event-by-event pile-up is much reduced. The $P / Z$ works properly up to $30-40 \mathrm{kcps}$ for mean energies up to $5-6 \mathrm{MeV}$.

For the 6-fold segmented detector configuration, a second amplifier serves as an output buffer and allows preamplifier conversion gains of $175 \mathrm{mV} / \mathrm{MeV}$ or $350 \mathrm{mV} / \mathrm{MeV}$, selectable by a solderable jumper. Two unipolar outputs with configurable polarity are provided.

The operational amplifier selected for the output stage, namely the LM6172, features low noise, low power and fast settling time. It has been chosen due to the overall power consumption limitation of the triple cryostat, with its 21 channels ( 3 detectors; each with a core and 6 segments).

The intrinsic rise time of the preamplifiers is about $\sim 13 \mathrm{~ns}(0 \mathrm{pF}$ detector capacitance and cold part at $\sim$ $150 \mathrm{~K}$ ) with a slope of $\sim 0.3 \mathrm{~ns} / \mathrm{pF}$ (detector capacitance seen in the position of the gate pin of the cold jFET) without noticeable overshoots or undershoots. The full technical specifications of the Miniball front-end electronics are shown in table 1.

In its standard way of cabling in the Miniball frame, the output signals from the preamplifiers are transmitted from the triple-cryostats to the remote fast ADCs of the digital electronics modules through $15 \mathrm{~m}$ coaxial cables, which makes the preamplifier assembly suitable for clean experimental environments.

\subsection{Pulse-shape analysis}

Before Miniball was constructed, pulse-shape analysis had already been investigated as a way to improve timing and peak-to-background ratios [8,13-15]. However, it was realized that this technique, when used with segmented Ge detectors, could yield position sensitivity. Being able to determine the location of the first interaction of a $\gamma$-ray with a Ge crystal makes it possible to divide up the crystal logically into pixels and perform the Doppler correction with the appropriate angle for each individual pixel. In this way, the granularity of the system is multiplied by the number of pixels per detector.

An idealized signal from a Ge detector can be thought of as a step function, to which the preamplifier adds an exponential decay. In real detectors, the finite drift times for electrons and holes through the crystal modify the rising flank of the signal. The time taken for this signal to reach its steepest slope is a direct measure of the drift time for the corresponding charge carriers and consequently of the distance those charge carriers had to migrate from the interaction point to the electrode. For the core electrode, 
in a detector with an approximately cylindrical geometry, this is an indication of the radius at which the interaction occurred. This method is referred to as the "time-tosteepest-slope method".

For segmented detectors such as those used by Miniball, there is an additional effect, as the electrons migrate to the positively biased core electrode and the holes migrate to the nearest segment electrode. If the interaction takes place close to the core electrode, the electrons arrive there before the holes arrive at the segment electrode, leading to a transient net positive charge. On the other hand, if the interaction takes place near to the segment electrode, a transient net negative charge is produced. These transient charges within one segment induce mirror charges on the adjacent segments, which are superimposed on any signal that might be in that segment. The amplitude of one of these mirror charges depends on the distance between the charge carriers and the segment boundary. Hence, by comparing the mirror charge amplitudes in segments at either side of the one in which the interaction took place, it is possible to determine the azimuthal position.

Miniball does not have any segmentation in the depth, so the depth of the interaction cannot be determined. This also limits the effectiveness of time-to-steepest-slope method for interactions at the front of the detector, where the assumption of cylindrical geometry is no longer valid.

At energies below $\approx 150 \mathrm{keV}$, the photoelectric effect dominates and there is only one interaction. For higher energies, however, there may be multiple interactions and for Doppler correction, it is the position of the first interaction which is needed, but it is not possible to determine which interaction occurred first. In this case, Miniball uses the concept of the "main interaction" (i.e. the one which deposits the most energy). For very high energies, pair production dominates and most of the energy is deposited in the first interaction. Even for Compton scattering with energies above $\approx 500 \mathrm{keV}$, most of the energy is still deposited in the first interaction. However, in the range from about 150 to $500 \mathrm{keV}$, Compton scattering often results in less than half the energy being deposited in the first interaction and then the main interaction is not the same as the first interaction [9]. Nevertheless, even in this case, the scattering angle may be small and the main interaction can still be at approximately the same angle as the first interaction. In other cases, the main interaction occurs in a position spatially close to the first interaction. So using the position of the main interaction for the Doppler correction still yields a considerable improvement compared to an unsegmented detector.

Unlike AGATA [16], Miniball does not attempt to determine the position of all interactions in three dimensions and perform tracking to determine which is the first one, but relies on the determination of the position of the main interaction in two dimensions. With the typical $\gamma$-ray multiplicities involved in experiments at REX-ISOLDE, it is not necessary to disentangle complex events due to many $\gamma$-rays.
Tests performed on a scanning table in Cologne using the prototype Miniball cluster yielded a spatial resolution of about $7.5 \mathrm{~mm}$, which is sufficient to define about 100 pixels per capsule [17], thereby increasing the granularity by two orders of magnitude. In an in-beam experiment at the Cologne tandem facility using a ${ }^{37} \mathrm{Cl}$ beam on a copper-backed deuterated titanium foil, the $2167.5 \mathrm{keV}$ transition of ${ }^{38} \mathrm{Ar}$ was observed by a Miniball detector placed $11 \mathrm{~cm}$ from the target, following the $\mathrm{d}\left({ }^{37} \mathrm{Cl},{ }^{38} \mathrm{Ar}\right) \mathrm{n}$ reaction. Using just the core positions to perform Doppler correction a FWHM of $35 \mathrm{keV}$ was obtained. This was reduced to $15 \mathrm{keV}$ by performing the correction for the angle of the segment which was hit and to $10.5 \mathrm{keV}$ by performing the full PSA [17]. The main contribution to this final resolution came not from the determination of the angle of the $\gamma$-ray, but from the energy and angle straggling of the ${ }^{38} \mathrm{Ar}$ ions, which were not measured [17].

On this basis, the eight triple clusters of the Miniball array, containing a total of $24 \mathrm{Ge}$ detectors can be placed in a compact geometry around a target chamber, providing maximum efficiency (comparable to EUROBALL), and still achieve a granularity twenty times better than EUROBALL. However, in order to exploit this granularity, a comparable precision in the determination of the angle of the particle, which emits the $\gamma$-ray, is also required. It is also necessary to know the speed of this particle and straggling in the target limits the accuracy, which can be achieved.

\subsection{Digital electronics}

As each of the 24 capsules in the eight Miniball triple cryostats provides signals from the core and each of the six segment electrodes, a system capable of acquiring data on 168 channels is needed. Furthermore, if pulse-shape analysis is to be performed, it is not sufficient to acquire just energy and time information, as additional information are needed to determine the position of the main interaction.

Miniball pioneered the use of digital electronics for $\gamma$ ray spectroscopy more than a decade ago, using a data acquisition system based on the Digital Gamma Finder with 4 Channels (DGF-4C) from the XIA company [12]. This is a single width CAMAC module providing four complete spectroscopic channels. After building up experience with earlier revisions, it was the revision D of the DGF-4C, which was adopted for use by Miniball.

The signals from each of the Miniball preamplifiers are passed directly to the DGF-4C modules, which contain an internal amplifier with programmable gain and offset and a Nyquist filter before a $40 \mathrm{MHz} 12$ bit sampling ADC. The filtering that is normally performed in an analog chain is performed digitally using algorithms implemented on field programmable gate arrays (FPGA), a fast filter being used for triggering, timing and pile-up rejection and a slow filter for energy determination. It is also possible to read out the traces via a FIFO, but although this feature is used for setting up the electronics, it is not used by Miniball during normal data acquisition. The FPGA determines when 
a trigger was detected, calculates some sums, which are later used to calculate the energy, and performs the pile-up rejection in real time using a pipeline design. This means that the FPGA can cope with high counting rates with no dead time and is essentially limited only by pile-up.

If a channel is validated at the end of the slow filter, its data are read out by the on-board digital signal processor (DSP), which calculates the energy including a ballistic deficit correction, from the sums provided by the FPGA. It also buffers the data into 8 Kword buffers and can histogram the data into $32 \mathrm{~K} 24$ bit spectra. Only validated data are processed in this way, and it is only the validated data which can give rise to internal dead time.

For Miniball the DGF-4Cs are used in pairs, with each pair of DGF-4Cs having the core signal and all six segments for a given capsule, leaving one channel unused. The core signal is used to generate a fast trigger, which causes the FPGAs for all seven channels to process the signal, even though there might be no signal on some segments. This is necessary, because the segments detect both signals and mirror charges and the latter can have either polarity and have quite small amplitudes. Moreover, their absence is just as important a piece of information as their presence, since this indicates that electron and hole drift times were equal. Both the fast and DSP validation triggers are shared between the two modules in a pair via a back-plane bus.

The DGF-4C provides the possibility for users to write their own code for the DSP in assembler. Miniball makes use of this feature to perform the pulse-shape analysis on board the DGF-4C [18]. For signals from the core, the time to steepest slope is calculated, while for the segments the mirror amplitudes are determined.

The DGF-4C keeps track of time by counting a $40 \mathrm{MHz}$ clocking signal with a 48 bit counter. Each of the 54 DGF$4 \mathrm{C}$ modules (48 for the signals from the Ge capsules and 6 used for timestamping other signals) has an oscillator, which can generate such a signal, but for Miniball an external $40 \mathrm{MHz}$ time reference is used. This is generated by a specially designed CAMAC module, which fans out the signal to all the DGF-4Cs in its crate and also passes the signal on to two repeater modules via Firewire, which in turn fan out the signal to the DGF-4Cs in the other two crates (three CAMAC crates are required for 48 DGF-4C modules). As all the DGF-4Cs count the same clocking signal they remain synchronous.

At the end of each beam spill (see sect. 4.1 for details about the timing structure at ISOLDE), the $8 \mathrm{~K}$ word buffers of each DGF-4C are read out using fast CAMAC. These buffers contain the energies of the $\gamma$-rays, 48 bit time stamps with $25 \mathrm{~ns}$ resolution and either the time to steepest slope or the mirror charge, for each of the 168 channels. This buffer size is large enough that the DGF$4 \mathrm{Cs}$ only need to be read out between beam bursts, so the readout does not produce additional dead time. Indeed, in normal operation at REX-ISOLDE, there is enough time between beam bursts to acquire a second off-beam buffer and read it out as well, since the accelerator repetition rate is of the order of $10 \mathrm{~Hz}$.
In order to determine the energy of a signal which occurs on the trailing edge of a previous signal but is well enough separated not to be considered as pile-up, it is necessary for the DGF-4C to know the exponential decay constant $\tau$ of the preamplifier accurately. Although this value is nominally $50 \mu \mathrm{s}$, there can be significant differences from detector channel to channel, as it is influenced by the $P / Z$ setting of the preamplifier. Consequently, it is necessary to scan the $\tau$ values to find the value which yields the optimum resolution at high count rate before starting to measure. For lower count rates, the effect of $\tau$ is less important as $\gamma$-rays are typically spaced further apart in time. The value of this characteristic decay constant only changes during maintenance, so the determination of $\tau$ only needs to be performed once at the beginning of a campaign of measurements.

The auxiliary detectors (see sect. 3) are read out using traditional analog electronics, although the ADCs and TDCs are capable of buffering up to 32 events. Consequently, a mechanism is needed to correlate events in the Ge detectors with those in the auxiliary detectors. This is done by using some extra DGF-4C modules, which acquire a TTL signal generated at the same time as the gate of the ADCs and the stops of the TDCs. In this way the $N$-th event in each ADC and TDC corresponds to the $N$-th event in the corresponding DGF-4C from where the time stamp can be obtained. Since 2009, the particle detectors were read out using ADC modules which provide this timestamp themselves using the same $40 \mathrm{MHz}$ signal as the DGF-4C modules as reference. Nevertheless, the TDCs and some special signals are still timestamped using additional DGF-4C modules. Using the time stamps, the data can be correlated offline.

\subsection{Data acquisition software}

The MAR $\mathrm{a}_{\mathrm{a}} \mathrm{B} \mathrm{U}$ [19] data acquisition system is used for all experiments with Miniball at REX-ISOLDE. It is based on the Multi Branch System (MBS) [20] as a front-end for data readout, event building and data transport. It runs on a PowerPC CPU under LynxOs. The back-end part is written within the ROOT framework [21] and provides class libraries for setup, run control, histogramming, data analysis and data storage. A variety of common VME and CAMAC modules are defined in $\mathrm{MAR}_{\mathrm{a}} \mathrm{B} \phi \mathrm{U}$ and can be easily implemented and configured with a user-defined configuration file. This configuration file describes the experimental setup containing definitions for hardware modules and histograms. From the configuration file, a code generator produces the full readout code in the MBS frontend and the main objects and functions to be used in the ROOT backend. The user supplies his tailor-made analysis code to be linked to the ROOT modules. Additionally, a dedicated graphical user interface exists to configure the DGF-4C modules. A display program provides tools to visualize the data either from the online data stream or offline from a raw data file. The latter is used for online monitoring of the experimental conditions and the overall performance of the setup. 


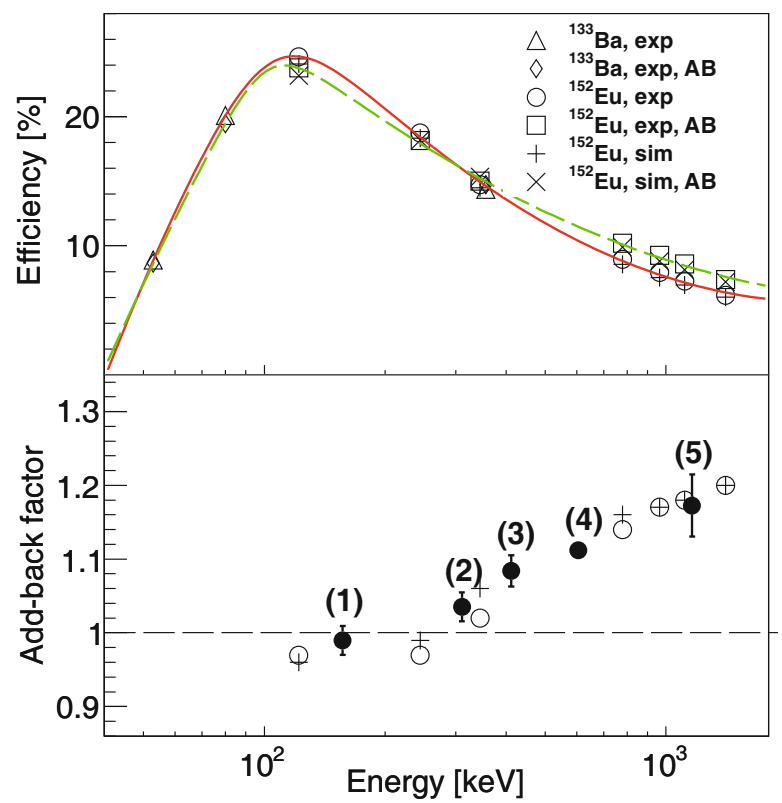

Fig. 4. (Color online) (Top) Measured and simulated photopeak efficiency for the Miniball array with the Coulomb excitation target chamber. Open symbols represent the experimental efficiencies determined using ${ }^{133} \mathrm{Ba}$ and ${ }^{152} \mathrm{Eu}$ sources, with and without an add-back (AB) algorithm applied. The crosses represent the simulated efficiencies. The solid line is a fit to the data without $\mathrm{AB}$ and the dashed line is a fit with $\mathrm{AB}$. Both the experimental and simulated values have been scaled to reproduce the absolute efficiency at $1.3 \mathrm{MeV}$ of $7.8 \%$ (with $\mathrm{AB}$ ) determined with a known ${ }^{60} \mathrm{Co}$ source. (Bottom) The ABfactors. The open symbols represent measured AB-factor for a ${ }^{152} \mathrm{Eu}$ source, whereas the crosses are the simulated AB-factors for ${ }^{152} \mathrm{Eu}$. The filled black symbols are AB-factors which were deduced from the intensities of transitions in radioactive ion beam experiments: (1) ${ }^{61} \mathrm{Mn}\left(7 / 2^{-} \rightarrow 5 / 2^{-}: 157 \mathrm{keV}\right)$ [22], $(2)+(3){ }^{109} \mathrm{Ag}$ (stable secondary target, $3 / 2^{-} \rightarrow 1 / 2^{-}: 312 \mathrm{keV}$ and $\left.5 / 2^{-} \rightarrow 1 / 2^{-}: 415 \mathrm{keV}\right),(4){ }^{74} \mathrm{Zn}\left(2^{+} \rightarrow 0^{+}: 606 \mathrm{keV}\right)[23]$ (5) ${ }^{120} \mathrm{Sn}$ (stable secondary target, $2^{+} \rightarrow 0^{+}: 1171 \mathrm{keV}$ ) [23].

\subsection{Efficiency and resolution}

In fig. 4 the measured full-energy peak efficiencies for the Miniball spectrometer in the Coulomb excitation configuration (see fig. $7(\mathrm{~A})$ ) are shown. The open symbols represent the measured efficiencies with point-like ${ }^{152} \mathrm{Eu}$ and ${ }^{133} \mathrm{Ba}$ intensity calibrated sources, positioned in the center of the spectrometer. The open circles and triangles represent the efficiencies when no add-back routine was applied to the data, the open squares and diamonds are the efficiencies when an add-back routine was applied. The application of an add-back (AB) routine involves the summing of the energies of two coincident gamma rays within $100 \mathrm{~ns}$ in neighboring cores on the same cluster detector. This situation corresponds to a Compton-scattered $\gamma$-ray event where the energy of the $\gamma$-ray is shared between two or more crystals in the same triple cluster detector. For higher-energy $\gamma$-rays, where scattering from one crystal into its neighbor is quite likely, this improves the efficiency, but for low-energy $\gamma$-rays, where scattering is less
Table 2. Set of parameters resulting from a fit to the experimental efficiencies with the parametrization of eq. (1). These coefficients correspond to the curves in fig. 4 .

\begin{tabular}{c|cc}
\hline & No add-back & With add-back \\
\hline$\alpha$ & 4.862 & 7.549 \\
$\beta_{0}$ & 25.669 & 24.987 \\
$\beta_{1}$ & 50.491 & 53.634 \\
$\beta_{2}$ & -37.034 & -19.954 \\
$\beta_{0}^{\prime}$ & 7.588 & 8.920 \\
$\beta_{1}^{\prime}$ & -10.031 & -10.053 \\
$\beta_{2}^{\prime}$ & 12.793 & 7.634 \\
\hline
\end{tabular}

likely, summing effects actually reduce the efficiency. For this reason a cut-off is normally applied and $\mathrm{AB}$ is only performed for energies above this threshold. A fit to typical experimental efficiencies is included, with the following parametrization:

$$
\begin{gathered}
\epsilon=\left\{\left[\sum_{i=0}^{2} \beta_{i}\left(\log _{10}\left(\frac{E}{100}\right)\right)^{i}\right]^{-\alpha}\right. \\
\left.+\left[\sum_{i=0}^{2} \beta_{i}^{\prime}\left(\log _{10}\left(\frac{E}{1000}\right)\right)^{i}\right]^{-\alpha}\right\}^{-\frac{1}{\alpha}},
\end{gathered}
$$

yielding the set of parameters presented in table 2 .

The ratio of the efficiency with $\mathrm{AB}$ to the efficiency without $\mathrm{AB}$ is referred to as the $\mathrm{AB}$-factor and is plotted in fig. 4. The AB-factor amounts up to $20 \%$ above $1 \mathrm{MeV}$. The open circles are the experimental AB-factors for ${ }^{152} \mathrm{Eu}$ and ${ }^{133} \mathrm{Ba}$. For some selected cases, the ABfactor obtained from transitions in Coulomb-excitation experiments with RIBs are given as well and they agree with the values measured with calibration sources. Relative efficiencies obtained from a GEANT4 simulation of the full spectrometer, scaled to reproduce the measured absolute efficiency at $1.3 \mathrm{MeV}$, are presented with the crosses and pluses in fig. 4. A good agreement is found between the experimental and simulated efficiencies.

The relative efficiencies for the T-REX configuration (see sect. 2.10.2) are similar, but the absolute efficiency with add-back at $1.3 \mathrm{MeV}$ is reduced to $5.0(3) \%$ [28] due to the increased detector-target distance imposed by the larger target chamber.

In fig. 5 the measured energy resolution of the Miniball spectrometer (sum of all 24 core spectra) is plotted. The crosses represent the full width at half maximum (FWHM) for the most intense transitions from the ${ }^{152} \mathrm{Eu}$ calibration source, when no $\mathrm{AB}$ procedure is applied to the spectra. The open circles represent the resolution, when an $\mathrm{AB}$ procedure is applied to the spectra. The diamonds and stars show FWHM values from the $\mathrm{d}\left({ }^{37} \mathrm{Cl},{ }^{38} \mathrm{Cl}\right) \mathrm{p}$ and $\mathrm{d}\left({ }^{37} \mathrm{Cl},{ }^{38} \mathrm{Ar}\right) \mathrm{n}$ reactions using just the Doppler correction at the segment level or with PSA, respectively [17]. In addition, the resolution of some selected Doppler-corrected transitions are plotted 


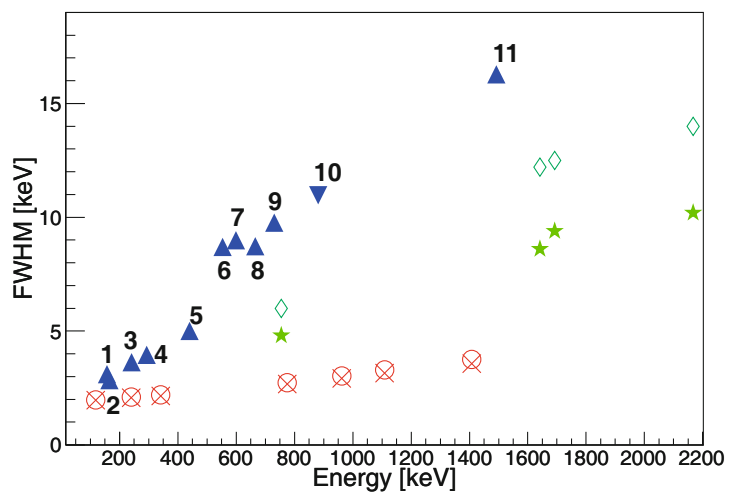

Fig. 5. (Color online) Energy resolution (FWHM) of the full Miniball array in the Coulomb excitation configuration. The red crosses are measured resolutions with a standard ${ }^{152} \mathrm{Eu}$ calibration source without $\mathrm{AB}$, while the red circles are with $\mathrm{AB}$. The green diamonds are from the $\mathrm{d}\left({ }^{37} \mathrm{Cl},{ }^{38} \mathrm{Cl}\right) \mathrm{p}$ and $\mathrm{d}\left({ }^{37} \mathrm{Cl},{ }^{38} \mathrm{Ar}\right) \mathrm{n}$ reactions at $1.9 \mathrm{MeV} / A[17]$ performing a Doppler correction at the segment level, while the green stars are the same data using PSA to determine the angle for the Doppler correction. The upward pointing blue triangles are the resolutions of the Doppler corrected transition in a nucleus (beam particle) which was Coulomb excited at $\approx 2.9 \mathrm{MeV} / A$. All these resolutions were obtained after add-back was applied and the Doppler correction was performed at the segment level. The downward pointing blue triangle, labeled (10), shows the resolution obtained after add-back and Doppler correction, where the $\gamma$ emission angle was determined after PSA. The numbered triangles correspond to the following transitions: (1) $7 / 2^{-} \rightarrow 5 / 2^{-}$at $157 \mathrm{keV}$ in ${ }^{61} \mathrm{Mn}$ [22], (2) $4_{1}^{+} \rightarrow 2_{1}^{+}$ at $166 \mathrm{keV}$ in ${ }^{224} \mathrm{Ra}$ [24], (3) $2_{1}^{+} \rightarrow 0_{1}^{+}$at $241 \mathrm{keV}$ in ${ }^{220} \mathrm{Rn}$ [24], (4) $4_{1}^{+} \rightarrow 2_{1}^{+}$at $293 \mathrm{keV}{ }^{220} \mathrm{Rn}$ [24], (5) $5 / 2_{1}^{+} \rightarrow 3 / 2_{1}^{+}$at $440 \mathrm{keV}$ in $\mathrm{d}\left({ }^{22} \mathrm{Ne},{ }^{23} \mathrm{Na}\right) \mathrm{n}$ calibration, (6) $2_{1}^{+} \rightarrow 0_{1}^{+}$at $554 \mathrm{keV}$ in ${ }^{96} \mathrm{Kr}[25],(7) 2_{1}^{+} \rightarrow 0_{1}^{+}$at $599 \mathrm{keV}$ in ${ }^{76} \mathrm{Zn}$ [23], (8) $2_{1}^{+} \rightarrow 0_{1}^{+}$ at $666 \mathrm{keV}$ in ${ }^{200} \mathrm{Po}$ [26], (9) $2_{1}^{+} \rightarrow 0_{1}^{+}$at $730 \mathrm{keV}$ in ${ }^{78} \mathrm{Zn}$ [23], (10) $2_{1}^{+} \rightarrow 0_{1}^{+}$at $885 \mathrm{keV}$ in ${ }^{32} \mathrm{Mg}$ [27], (11) $2_{1}^{+} \rightarrow 0_{1}^{+}$at $1492 \mathrm{keV}$ in ${ }^{80} \mathrm{Zn}[23]$.

with filled triangles. The upwards pointing triangles are resolutions obtained from Doppler corrected transitions in the beam-like nucleus, where the angular coordinates of the $\gamma$-rays were determined on segment level and the angular coordinates of the detected beam-like particles were deduced from the angular and sector strips of the Double-Sided Silicon-Strip Detector (DSSSD, see sect. 3). In the case of the downward pointing triangle, the angular coordinates of the $\gamma$-rays were obtained from the on-board PSA. The latter resolution was obtained from Coulomb excitation of ${ }^{32} \mathrm{Mg}$ [27]. More details on the Doppler correction procedure are given in sect. 7 .

\subsection{Control systems at Miniball}

In order to achieve a compact geometry, the Miniball clusters have relatively small Dewars. As a result, the $\mathrm{LN}_{2}$ holding time of the clusters is only about 12 hours. Furthermore, the 21 FETs in the cold part of the detector generate a significant amount of heat $(\approx 1 \mathrm{~W})$, which has

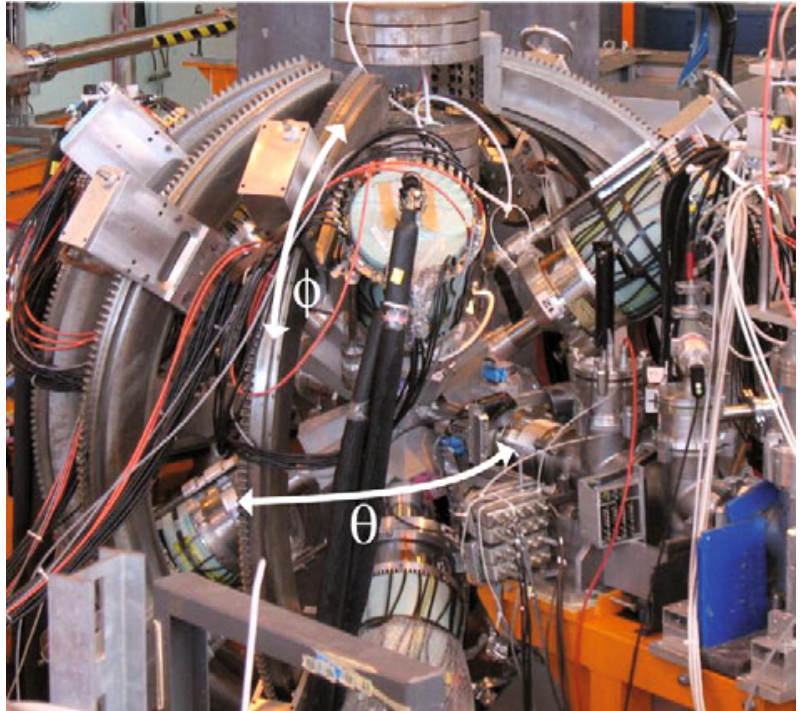

Fig. 6. (Color online) The Miniball frame is built in two halves, which can be slid apart on rails to provide access to the target chamber (not shown). Each half has three toothed arcs, which can rotate about a vertical axis, on which the clusters are mounted. The cluster mountings have gears which interlock with the teeth of the arcs, so each cluster can be moved up and down the arc in a controlled manner. The cluster mountings have rods, which enable the individual clusters to be moved in and out relative to the target. The clusters can be rotated about their axis.

to be compensated by liquid nitrogen cooling. This reduces the holding time to about eight hours. Consequently an automated $\mathrm{LN}_{2}$ filling system is required.

The $\mathrm{LN}_{2}$ filling system originally designed for EXOGAM was adapted for Miniball and new software was written for this system. The system fills every six hours and continually monitors the temperatures of the detectors using a PT100 located next to one of the three capsules inside each triple cryostat and the system can react to a temperature rise by performing an emergency fill, ramping down the high voltage and/or alerting users by SMS text message to a mobile telephone.

In order to protect the system against inevitable power failures, the computer controlling the filling system is connected to an uninterruptible power supply (UPS), which can deliver power to the computer and HV system for up to 3 hours.

The high voltage mainframe which supplies the bias voltage for all 24 capsules is also powered from the UPS and controlled from the same computer using software written specially for Miniball. In the event of a power failure or a problem with the liquid nitrogen filling, the computer can ramp down the high voltages safely.

\subsection{Detector frame}

In order to provide the maximum flexibility for different kinds of Miniball experiments, performed at different facilities, a flexible frame was designed (see fig. 6), which allows 
for a wide range of configurations, allowing for each detector to be placed at variable angle, distance and orientation with respect to the target. An inner ring is bolted around each Miniball cluster, which is sandwiched between two outer rings, which are bolted together and mounted onto the frame. Teflon pieces, at the points where the ring attached to the cluster touches the other two, ensure that the cluster can rotate freely about its axis.

The outer rings are mounted on rods, which allow the whole assembly to slide in and out, which, since 2009 , can be adjusted by rotating an additional threaded rod. In this way it is possible to adjust the distance between the detectors and the target for each cluster individually. These rods are fixed to riders which can slide along one of six arcs (three on each side). The arcs have teeth and the riders have gears, so that the cluster assembly can be moved up and down the arcs (corresponding to a rotation about the beam axis) by turning a wheel. The arcs can rotate about the vertical axis of the spectrometer.

The arcs are mounted on two bases, one either side of the target chamber, which can be slid in and out perpendicular to the beam direction, making it possible to move all the detectors on each side back to provide access to the target chamber. When the spectrometer is closed, locking pins make the whole system rigid and also ensure that the two halves of the frame are grounded together.

\subsection{Target chambers}

Two target chambers are available for experiments at REX-ISOLDE: one for Coulomb-excitation experiments and the other for transfer reactions.

\subsubsection{Target chamber for Coulomb excitation}

The target chamber used in Coulomb-excitation experiments, surrounded by the eight Miniball clusters is shown in fig. 7(A). It consists of a thin-walled hollow sphere of inner radius $\approx 80 \mathrm{~mm}$ machined out of a single piece of $\mathrm{AlMg}_{3}$. Inside this chamber a target wheel, that can accommodate six different targets, can be mounted (see fig. 7(B)). The particle detector used for the Coulombexcitation experiments is shown in fig. $7(\mathrm{C})$ and will be described in sect. 3 . It is positioned $25-31 \mathrm{~mm}$ from the target wheel (in forward direction) and is mounted on three fixed mounting rods (see fig. $7(\mathrm{~B})$ ). There is limited flexibility in the positioning of the detector because of the limited space in the target chamber. The flat cables in fig. 7(B) connect to the particle detector and are guided through the beamline to the feedthrough connections downstream from the target chamber. Each cluster detector resides at an average distance of $\approx 10 \mathrm{~cm}$ from the center of the target chamber. This spherical configuration ensures a coverage of $\approx 60 \%$ of $4 \pi$ around the reaction target (see fig. $7(\mathrm{~A})$ ). The angular position of each detector is approximately $45^{\circ}$ (for the forward detectors) or $135^{\circ}$ (for the backward detectors) with respect to the beam line $(\theta)$.
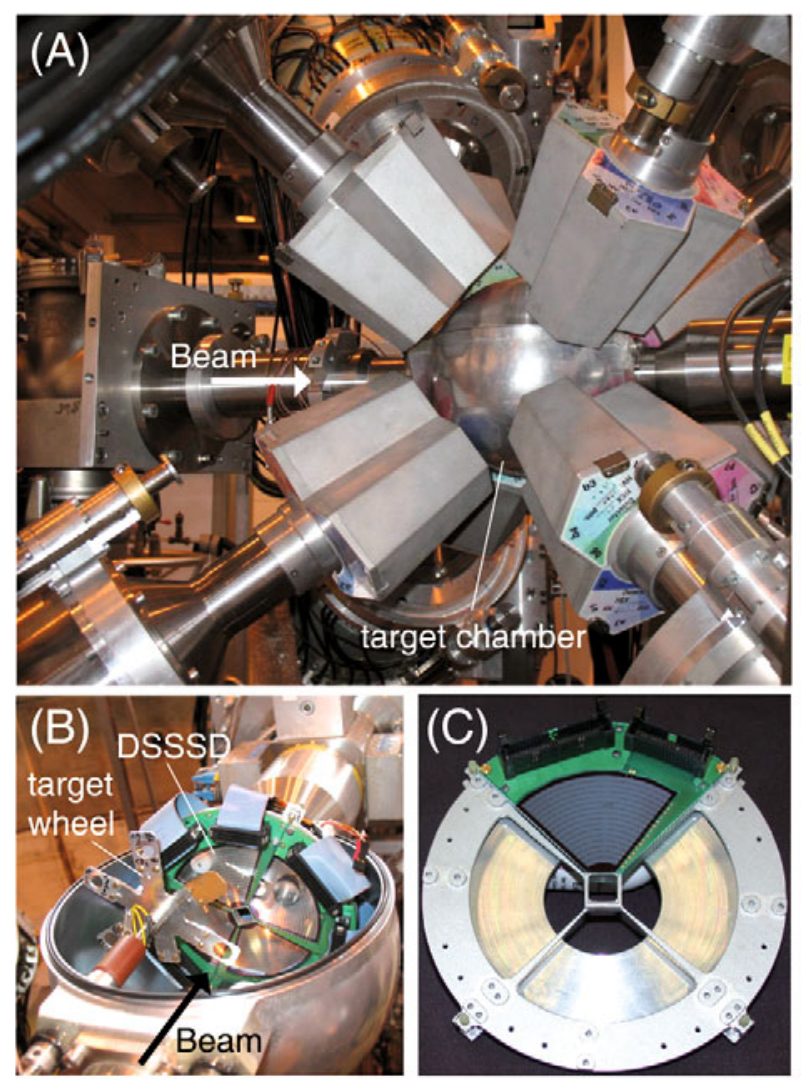

Fig. 7. (Color online) (A) Target chamber in the Coulomb excitation setup. (B) Detail of the target chamber inside with the upper part removed. (C) A quadrant of the particle detector used in Coulomb excitation experiments (DSSSD — see sect. 3).

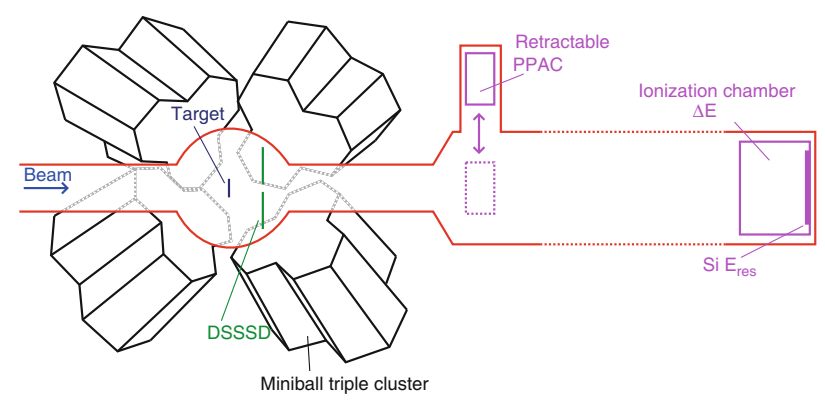

Fig. 8. (Color online) Schematic diagram of Coulomb excitation setup showing half of the Ge detectors around the target chamber containing the DSSSD. The PPAC may be moved into position or retracted to allow the beam through to the ionization chamber (see text for a description of the various parts).

In the $\phi$ direction, the four clusters at forward and backward angles are positioned roughly on a circle, separated by $90^{\circ}$. Figure 8 illustrates the setup schematically, with the four clusters on the left side with respect to the beam direction, shown. 

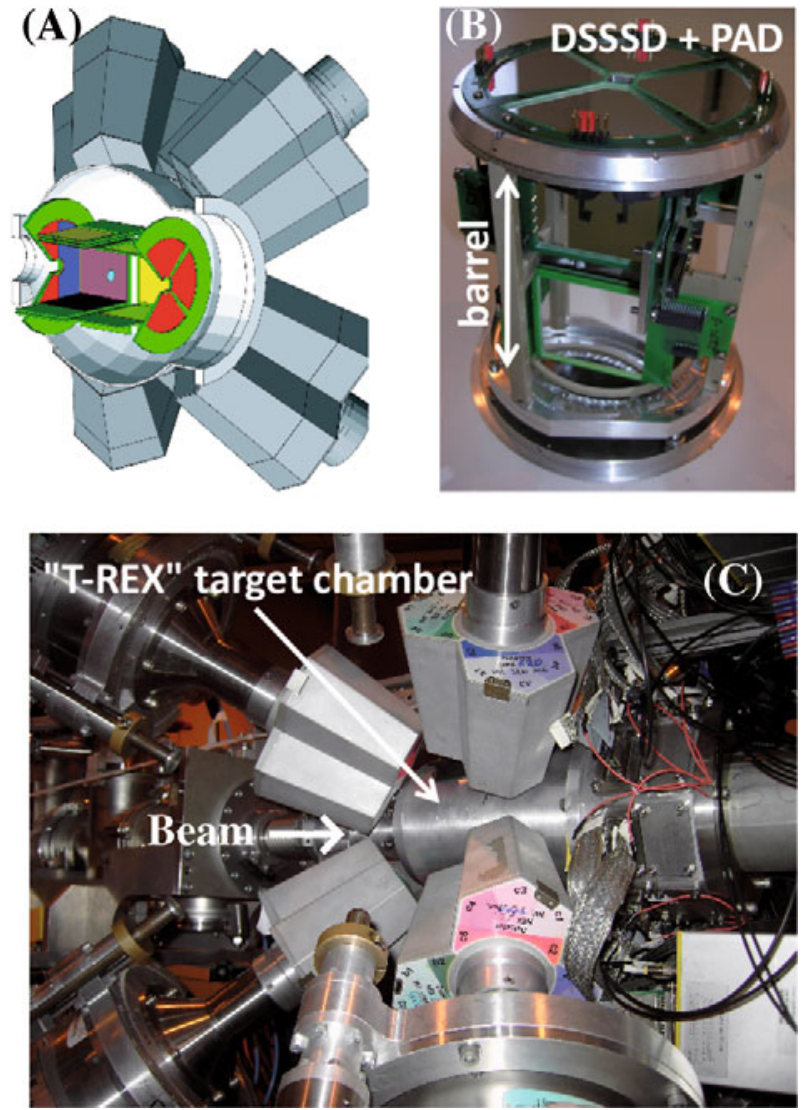

Fig. 9. (Color online) (A) The "T-REX" setup used in GEANT4 simulations. (B) Detail of the barrel-shaped detector for particle detection (see sect. 3). (C) Final configuration of the "T-REX" setup at REX-ISOLDE in 2008.

\subsubsection{Target chamber for transfer reactions}

A second target chamber was built in 2007 in order to extend the physics program at REX-ISOLDE to transfer reactions in inverse kinematics. This so-called "T-REX" target chamber was simulated in detail with GEANT4 [29] to optimize the design for maximum efficiency for both particle and $\gamma$ detection $[28,30]$. The layout used in the simulation is shown in fig. 9(A). A picture of the constructed barrel-shaped particle detector is shown in fig. 9(B) and will be described in sect. 3 . The cylindrical shape of the "T-REX" chamber forces the clusters in forward direction to be positioned at slightly larger distances from the secondary target. A picture of the final "T-REX" configuration is shown in fig. $9(\mathrm{C})$.

\section{Particle detectors}

\subsection{Coulomb excitation}

For Coulomb-excitation experiments, four DSSSD quadrants, are used, typically with a thickness of $\approx 500 \mu \mathrm{m}$, though detectors down to $40 \mu \mathrm{m}$ have been used with heavier beams. This detector stops all the scattered heavy ions (beam- and target-like) in the angular range covered by the DSSSD, for all combinations of post-accelerated

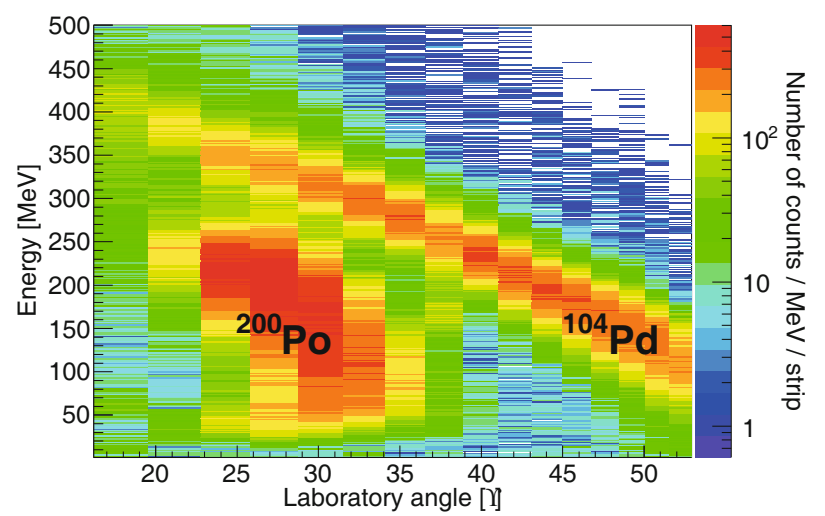

Fig. 10. (Color online) Spectrum measured with the DSSSD with a $2.85 \mathrm{MeV} / A{ }^{200} \mathrm{Po}$ beam incident on a $2.0 \mathrm{mg} / \mathrm{cm}^{2}$ ${ }^{104} \mathrm{Pd}$ target $[26,37]$. The spectrum is conditioned by coincident detected beam-like particles $\left({ }^{200} \mathrm{Po}\right)$ and target-like ejectiles $\left({ }^{104} \mathrm{Pd}\right)$. The width of the bins on the $x$-axis corresponds to the width of a strip.

ion beams and reaction targets which have been used so far at REX-ISOLDE (see table 3). This type of detector was described extensively in [31] and a summary is presented here. A picture of the DSSSD configuration is shown in fig. $7(\mathrm{C})$, where only one quadrant is installed. The front side of one quadrant is divided in 16 annular strips (rings) with $1.9 \mathrm{~mm}$ strip width and $2.0 \mathrm{~mm}$ pitch. The innermost strip has an inner radius of $9 \mathrm{~mm}$. The average distance between the secondary Coulomb excitation target and the DSSSD is $31(1) \mathrm{mm}$. Thus, the angular coverage in $\theta$ by the DSSSD spans from $\approx 16^{\circ}$ up to $\approx 53^{\circ}$ with a geometrical uncertainty on the angular coverage of $0.5^{\circ}$ for the inner and $1^{\circ}$ for the outer annular strips. The back side of one quadrant is divided in 24 sectors with an angular range of $3.4^{\circ}$ per sector. Most of the Coulomb excitation experiments are performed with pairwise coupled sector strips, effectively reducing the angular resolution in $\phi$ to $7.8^{\circ}$. The energy resolution of the DSSSDs at $5 \mathrm{MeV}$ is around $70 \mathrm{keV}$ when new and around $100 \mathrm{keV}$, after half a year of operation inbeam. Elastic scattering of ${ }^{22} \mathrm{Ne}$ or ${ }^{40} \mathrm{Ar}$ stable ion beams from the REXTRAP-REXEBIS combination (see sect. 4) at $2.9 \mathrm{MeV} / A$ is used to calibrate the DSSSD beyond $5 \mathrm{MeV}$.

Optionally, four $\approx 1 \mathrm{~mm}$ thick unsegmented Si detector quadrants ( $E$-detectors) can be mounted behind the DSSSDs, in order to form a $\Delta E-E$ telescope to identify light particles (such as protons from inverse $(d, p)$ reactions) which punch through the $500 \mu \mathrm{m}$ DSSSDs. This reduces the distance between the target and the DSSSD by about $6 \mathrm{~mm}$, changing the angular range to $\approx 20-59^{\circ}$. The 29 (= 16 front strips +12 back strips +1 E-detector $)$ channels for each quadrant are sent to RAL108 chargesensitive preamplifiers and subsequently to RAL109 shaping/discriminator amplifiers [32-34]. The 29 amplified and shaped signals (rise time of $1 \mu \mathrm{s}$ ) are input to one CAEN V785 peak sensing ADC [35] (until 2008) or one Mesytec MADC [36] (from 2009 onwards).

An exemplary spectrum, measured with the four DSSSD quadrants, is shown in fig. 10. The spectrum was 
obtained with a $2.85 \mathrm{MeV} / A{ }^{200} \mathrm{Po}$ beam, incident on a $2.0 \mathrm{mg} / \mathrm{cm}^{2}{ }^{104} \mathrm{Pd}$ target. This spectrum was conditioned by coincident beam-like particles and target-like recoil particles in the DSSSD, which are emitted back to back in the center-of-mass frame. Two distinct regions can be disentangled: in the upper region are the recoiling ${ }^{104} \mathrm{Pd}$ nuclei, whereas in the lower region are the scattered ${ }^{200} \mathrm{Po}$ nuclei.

It should be noted that the pulse-height defect due to a combination of the stopping by nuclear collisions, incomplete charge collection and surface dead layers can be important for heavy ions, so an energy calibration must be performed with the same beam. However, for most purposes, only the angles and a separation between target and projectile ions is required and the energy may be deduced from the kinematics.

\subsection{Transfer reactions}

A dedicated new array of position-sensitive silicon detectors covering a larger angular range in $\theta$ has been designed and built in 2007 for transfer experiments at REXISOLDE $[28,30]$.

The T-REX array consists of two $\approx 500 \mu \mathrm{m}$ DSSSDs: one in forward and one in backward direction, and a barrel of eight position-sensitive planar detectors, positioned symmetrically around $90^{\circ}$ (see fig. 9(B)). Both the forward and backward DSSSDs are combined with $500 \mu \mathrm{m}$ unsegmented $E$-detectors in order to form $\Delta E$ - $E$ telescopes to identify different reaction products. In forward direction these can be the different light ejectiles from the target $(\mathrm{p}, \mathrm{d}, \mathrm{t}, \alpha)$ and heavier isotopes, such as beam-like particles or the heavy carrier material of the target. The DSSSDs are connected to MUX-32 modules from Mesytec [38], which provide a multiplexed output, effectively reducing the amount of output channels to four: two for energy and two for positional information. The accompanying unsegmented $E$-detectors are connected to MSI- 8 preamplifier and integrated shaper and timing filter amplifier modules [39].

The forward and backward barrel detectors are identical and consist of a stack of $140 \mu \mathrm{m}$ and $1000 \mu \mathrm{m}$ planar Si detectors. The thinner Si detectors are segmented in 16 strips perpendicular to the beam axis. Positional information along these strips is obtained from the charge division on a resistive layer. The thicker Si detectors are not segmented. In forward direction, this stack of Si detectors serves as a $\Delta E-E$ telescope to identify the different ejectiles from the target. All detectors in forward direction are protected by a thin foil, which stops beam particles scattered by the carrier material of the target (e.g., carbon, titanium, ... ). For reactions with a beam lighter than the carrier material also the backward detectors are equipped with a protection foil.

The thin $\Delta E$ barrel detectors are connected to MPR16 charge-sensitive preamplifiers [40], combined with STM-32 [41] shaper amplifiers. The accompanying Edetectors are connected to MSI-8 preamplifiers. All shaped

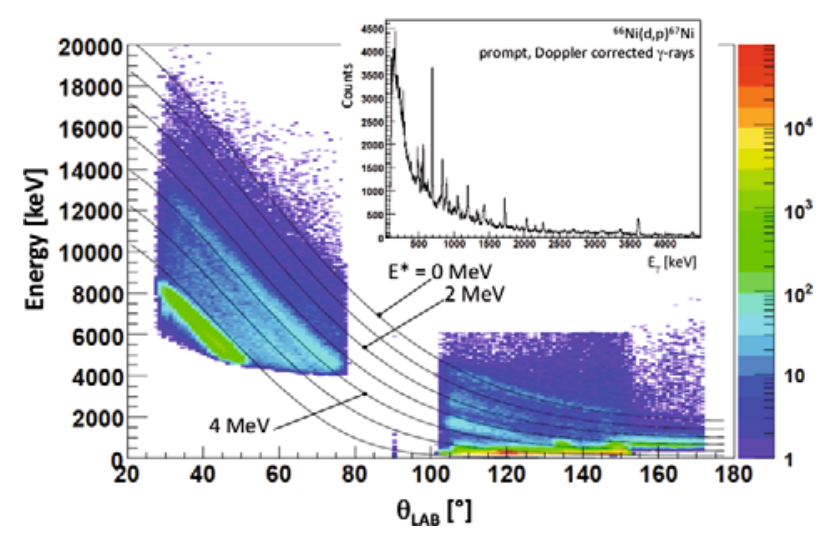

Fig. 11. (Color online) Proton spectrum observed with the "T-REX" silicon detector setup during the $\mathrm{d}\left({ }^{66} \mathrm{Ni},{ }^{67} \mathrm{Ni}\right) \mathrm{p}$ experiment [43]. The inset shows the prompt $\gamma$-spectrum coincident with the recoiling protons.

energy signals and position signals from the MUX-32 modules are sent to VME MADC modules, where internal time stamping is performed using the same time base as the DGF-4C modules. The energy resolution for the protons ranges from $250 \mathrm{keV}$ to $2 \mathrm{MeV}$, depending on their scattering angle, energy and thickness of the target. The coincident $\gamma$-rays have to be used in order to identify the populated final state. By gating on a $\gamma$-ray which depopulates the state, it is possible to deduce the angular distribution of the emitted protons which correspond to the excitation of that state. The angular resolution is typically better than $5^{\circ}$.

In fig. 11, a proton spectrum is shown, measured with the $\mathrm{d}\left({ }^{66} \mathrm{Ni},{ }^{67} \mathrm{Ni}\right) \mathrm{p}$ reaction [42]. This spectrum incorporates the energies measured in the backward DSSSD and all the barrel detectors and was obtained with a $10 \mu \mathrm{m}$ polyethylene target. In the inset, the prompt $\gamma$-spectrum coincident with the recoiling protons is shown, indicating the wealth of spectroscopic information obtained from this experiment. The different excited states populated in the inverse $(d, p)$ reaction can be disentangled from the proton spectrum, as indicated in fig. 11.

In backward direction, the protons from the inverse $(\mathrm{d}, \mathrm{p})$ transfer reaction have sufficiently low energy (from $100 \mathrm{keV}$ up to few $\mathrm{MeV}$ ) to be stopped in the first segmented detector. The $E$-detector can be used as veto to subtract the electron background of $\beta$-decay origin from the low-energy proton spectra. Without this possibility to veto the events in the thin detector, the spectrum of low-energy protons is heavily contaminated by the energy deposited in the thin detector by the electrons. Since 2011, a voltage has been applied to the target ladder in order to reduce the number of $\delta$ electrons that hit the detectors.

The first experiments successfully performed with this setup, in the last four years, addressed the nuclear structure of neutron-rich nuclei at the "shore" of the "island of inversion" using the $\mathrm{d}\left({ }^{30} \mathrm{Mg},{ }^{31} \mathrm{Mg}\right) \mathrm{p}$ and $\mathrm{t}\left({ }^{30} \mathrm{Mg},{ }^{32} \mathrm{Mg}\right) \mathrm{p}$ [44] reactions. These pioneering experiments were followed by studies of $\mathrm{d}\left({ }^{66} \mathrm{Ni},{ }^{67} \mathrm{Ni}\right) \mathrm{p}$ [45], $\mathrm{d}\left({ }^{78} \mathrm{Zn},{ }^{79} \mathrm{Zn}\right) \mathrm{p}[46]$ and $\mathrm{t}\left({ }^{44} \mathrm{Ar},{ }^{46} \mathrm{Ar}\right) \mathrm{p}[47]$ transfer reactions. 


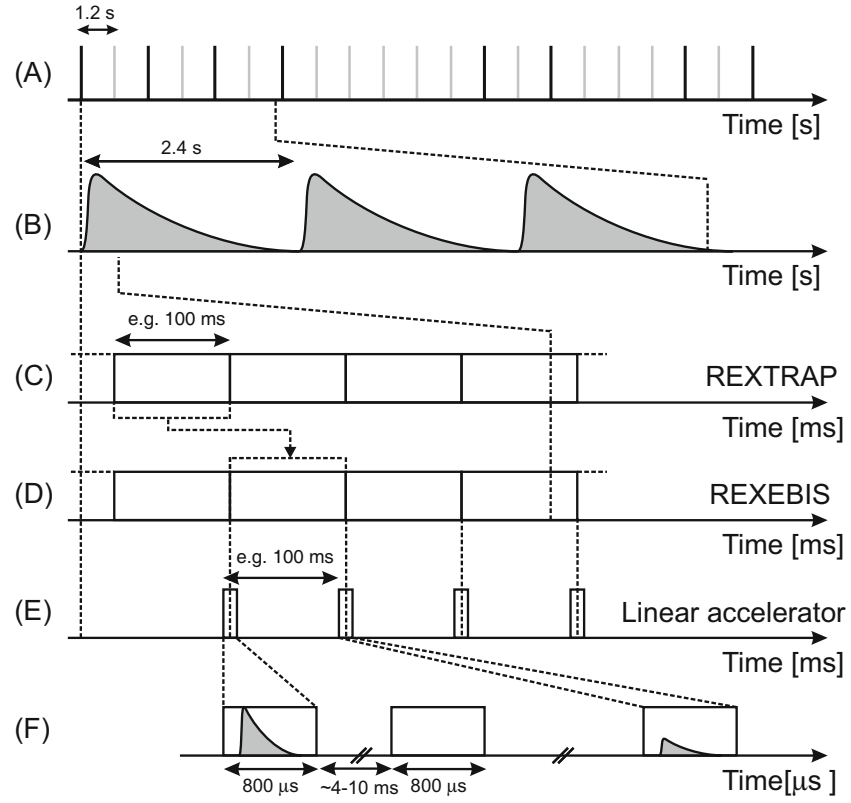

Fig. 12. (A) The proton bunches from CERN's PSB, organized in the so-called "supercycles" separated by 1.2 seconds, which are distributed to the different experiments. The proton bunches to the ISOLDE primary target are indicated in black, while those going to other experiments are in gray. (B) Release profile of produced isotopes from the primary target. The time constants of such a release curve depends on the type and settings of the ion source, the chemistry of the element and the half-life of the isotope in question. (C-D) REXTRAP and REXEBIS beam bunches. (E) RF window of the REX linac. (F) "on-beam" and "off-beam" time windows using the Miniball setup.

\section{Trigger scheme and timing properties}

The specific timing properties and the production mechanisms at the (REX-)ISOLDE facility have a major impact on the sensitivity and selectivity which is achievable with the Miniball setup. In the following, the detailed time structure of REX-ISOLDE is discussed together with the implications it has on data taking at the Miniball setup.

\subsection{Specific timing at ISOLDE}

The relevant time scales and time structures are given in fig. 12. The "driver beam" of ISOLDE is a $1.4 \mathrm{GeV}$ proton beam delivered by the Proton Synchrotron Booster (PSB) at CERN. The PSB provides proton bunches of $\approx 100 \mu \mathrm{s}$ spaced by $1.2 \mathrm{~s}$, which are distributed to the various CERN experiments. These bunches are organized in a so-called "supercycle", consisting of a few dozen bunches, which defines the order in which the bunches are sent to different experiments. In general, ISOLDE can utilize around $50 \%$ of these bunches, which corresponds to about $2 \mu \mathrm{A}$. In the example shown in fig. 12, it was requested to have at least $2.4 \mathrm{~s}$ between bunches on the ISOLDE primary target, in order to accommodate the slow release of the isotope of interest. With each impact of a proton pulse

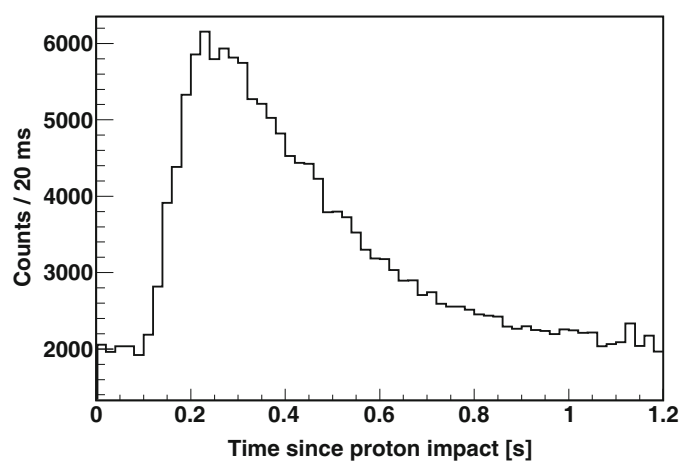

Fig. 13. Experimental release profile of the $A=80$ beam, produced with a $\mathrm{UC}_{x}$ target and the RILIS laser ion source. This release profile contains laser ionized $\mathrm{Zn}$ isotopes and the surface ionized $\mathrm{Ga}$ and $\mathrm{Rb}$ isotopes. The offset of $\approx 90 \mathrm{~ms}$ between the proton impact and the first detected particles in the Miniball setup at the start is due to the summed trapping and charge-breeding times (both $\approx 45 \mathrm{~ms}$.)

on the primary target, radioactive isotopes are produced by either fission, spallation or fragmentation of a variety of primary target materials. In $\approx 70 \%$ of the cases this is proton induced fission of ${ }^{238} \mathrm{UC}_{x}$. The radioactive isotopes diffuse through the thick primary target towards a transfer line, where potentially some chemical selectivity prevents undesired species from diffusing into the ion source (for an example on Rb suppression, see [48]). The ionization of the desired chemical element takes place in either the transfer line (surface and laser ion source) or just after the transfer line (plasma ion source). A review of target ion-source technology can be found in [49]. The target-ion source combination is heated between 700 and $2000^{\circ} \mathrm{C}$ to decrease the diffusion time. Though, the high temperature increases the probability for surface ionization, which is in most cases undesirable. The ionized species $\left(1^{+}\right.$charge state) are extracted from the target-ion source by applying an electrostatic potential, which is typically $30 \mathrm{kV}$ for REX-ISOLDE experiments. After mass separation, the isotopes are guided towards the REX-ISOLDE experiment. In figs. 12(B) and 13, characteristic release profiles of the mass-separated isotopes are shown. In fig. 13 the release profile is the superposition of the fast release of ${ }^{80} \mathrm{Zn}\left(T_{1 / 2}=545(16) \mathrm{ms}\right)$ and the slower release of the contaminating ${ }^{80} \mathrm{Ga}$ and ${ }^{80} \mathrm{Rb}$. In this time spectrum, the counts correspond to detected scattered particles in the DSSSD at the Miniball setup. A detailed description of this particular release profile can be found in [23]. The release time profile varies largely from one element to the other and this can be exploited to enhance the signal-tobackground ratio, as will be shown in sect. 6.4. A detailed description of release characteristics at ISOLDE can be found in [50].

Before post-acceleration to $\approx 3 \mathrm{MeV} / A$, the mass separated radioactive isotopes are charge bred from $Q=1^{+}$ to higher charge states $\left(\mathrm{n}^{+}\right)$in order to meet the injection requirement of the acceleration cavities (in particular the interdigital H-structure (IHS)), which requires that 


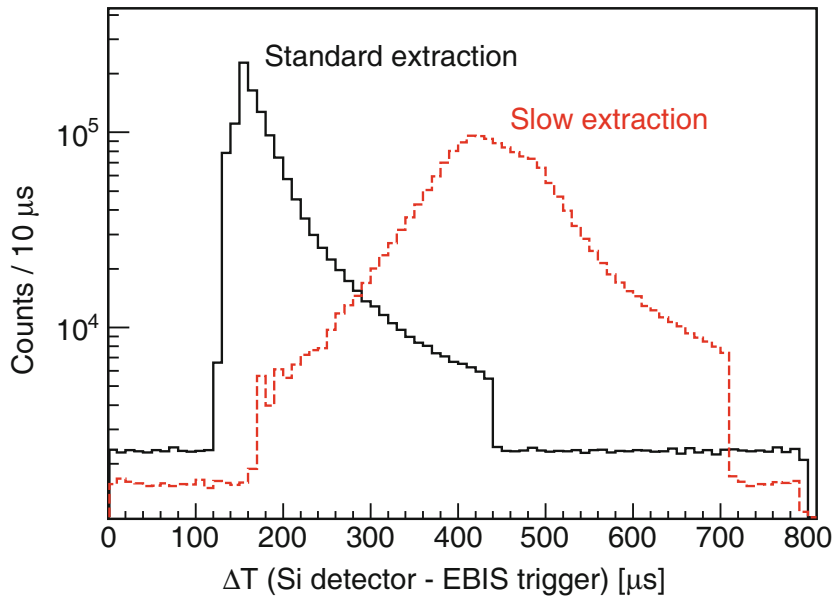

Fig. 14. (Color online) Illustration of the "standard" (solid black lines) and "slow extraction" (dashed red) modes of the REXEBIS. These time spectra are obtained from scattered particles in the barrel detector during the $\mathrm{d}\left({ }^{66} \mathrm{Ni},{ }^{67} \mathrm{Ni}\right) \mathrm{p}$ experiment.

$A / Q<4.5$ [1]. This is done in two stages: firstly, the released isotopes are accumulated in a Penning trap (REXTRAP) [51], where they are cooled and stored for a period ranging from $10 \mathrm{~ms}$ up to some hundreds of ms. This period depends on the desired charge state, and thus for a given mass, the $A / Q$ ratio. For heavier isotopes longer charge-breeding times are needed. The cooling in REXTRAP is mass dependent and potentially some additional mass selectivity can be achieved in REXTRAP, in order to purify the radioactive beam from stable isobaric contaminants [52]. Secondly, the accumulated and cooled isotopes are transmitted to the REX Electron Beam Ion Source (REXEBIS) [53-55] in a short time period of some $10 \mu \mathrm{s}$. The isotopes spend the same period in the REXEBIS charge breeder. Both trapping and charge breeding periods are schematically shown in fig. $12(\mathrm{C}-\mathrm{D})$.

The linear accelerator is triggered by the REXEBIS some $100 \mu$ s prior to injection of the isotopes into the linac and the RF cavities are on during $800 \mu \mathrm{s}$ (see fig. 12(E)). In "standard" operation, the ions are extracted from the REXEBIS in a pulse with a total width of about $300 \mu \mathrm{s}$, as shown in fig. 14. The bulk of the isotopes is released in about $100 \mu$ s only. In the next section it will be shown that this bunched structure, puts limitations on the manageable incoming beam intensities. In the "slow" extraction mode, the ion pulse is about $500 \mu$ s wide and the peak intensity is spread over about $300 \mu$ s as shown in fig. 14 . The "slow" extraction is used for high-intensity beams, where the pile-up in the particle detectors would be too large otherwise. In the transfer reaction setup, the highintensity beams cause a considerable noise in the barrel detectors, which can be largely suppressed by using this "slow extraction mode". The time spectra in fig. 14 contain the number of scattered particles in the barrel detector during the $\mathrm{d}\left({ }^{66} \mathrm{Ni},{ }^{67} \mathrm{Ni}\right) \mathrm{p}$ experiment as a function of time after the linac was triggered. The solid and dashed curves illustrates the standard and slow extraction mode, respectively. The different offset of about $50 \mu$ s has no real

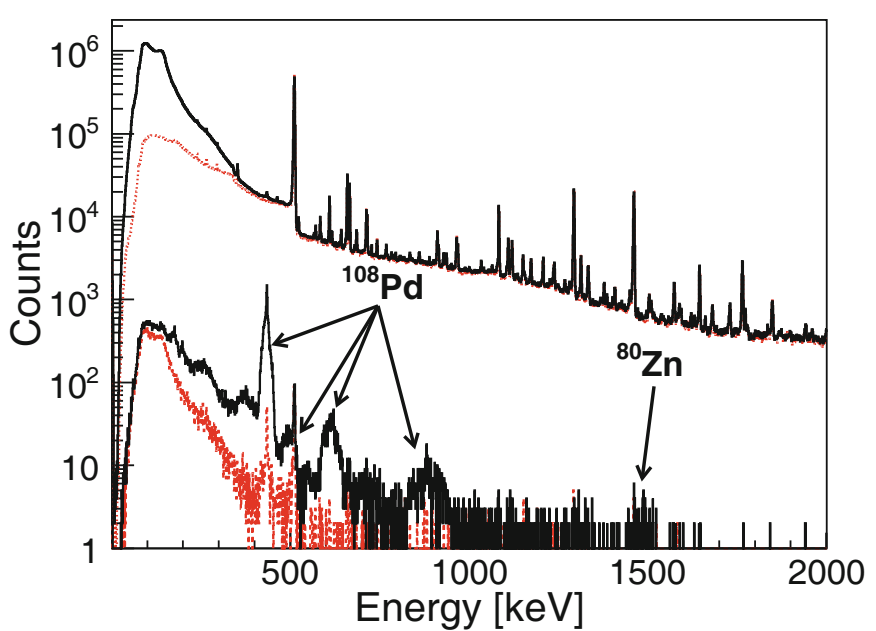

Fig. 15. (Color online) The upper plot represents the measured $\gamma$-ray singles Miniball spectra during beam-on windows (solid black lines) and beam-off windows (dashed red). The lower plot represents the in-beam Miniball $\gamma$-ray spectra, when additional prompt (solid black lines) and random (dashed red) conditions are requested with scattered beam- or target-like particles. These spectra are not Doppler corrected and were measured in a Coulomb-excitation experiment of ${ }^{80} \mathrm{Zn}$ on a ${ }^{108} \mathrm{Pd}$ target [23].

consequence, since the total injection time of particles in the linac is covered in both cases by the $800 \mu \mathrm{s} R F$ window. The counts before and after the release profile originate from $\beta$-decay electrons and are not related to the incoming beam (i.e. the inverse (d, p) transfer reaction).

During the full $800 \mu \mathrm{s}$ RF window, the data acquisition system registers all the information in the Miniball array and the particle detectors. During this time window, which is referred to as the "beam-on" window, the beam collides with the secondary target. In the case of Coulombexcitation experiments, this is typically a thin foil of $1-$ $4 \mathrm{mg} / \mathrm{cm}^{2}$ of a stable isotope. In the case of transfer reactions, the foil typically consists of $10-100 \mu \mathrm{m}$ of deuterated polyethylene or tritium-loaded titanium foils. Since it is crucial to identify all sources of background during the "beam-on" window, a second time window of $800 \mu$ s (see fig. 12(F)) opens 4-10 ms after the "beam-on" window, during which time there is no beam from the accelerator. This will be referred to as the "beam-off" window. The 4$10 \mathrm{~ms}$ time delay between beam-on and beam-off windows is the typical time needed for the data acquisition system to read the buffered data from the electronics modules and transfer it to disk after the beam pulse. During the "beamoff" window, the data acquisition system stores again all information detected in Miniball and particle detectors. Note that only $\beta$-decay electrons or $\alpha$-decay following the decay of $\alpha$-emitters will be detected in the particle detectors during this time window. The Miniball registers the remaining radioactivity in the target chamber, originating from the implanted beam particles.

In fig. 15 spectra recorded during "beam-on" windows (solid) and "beam-off" windows (dashed) are superimposed (the upper spectra in fig. 15). The spectra were measured during a Coulomb-excitation experiment with a 

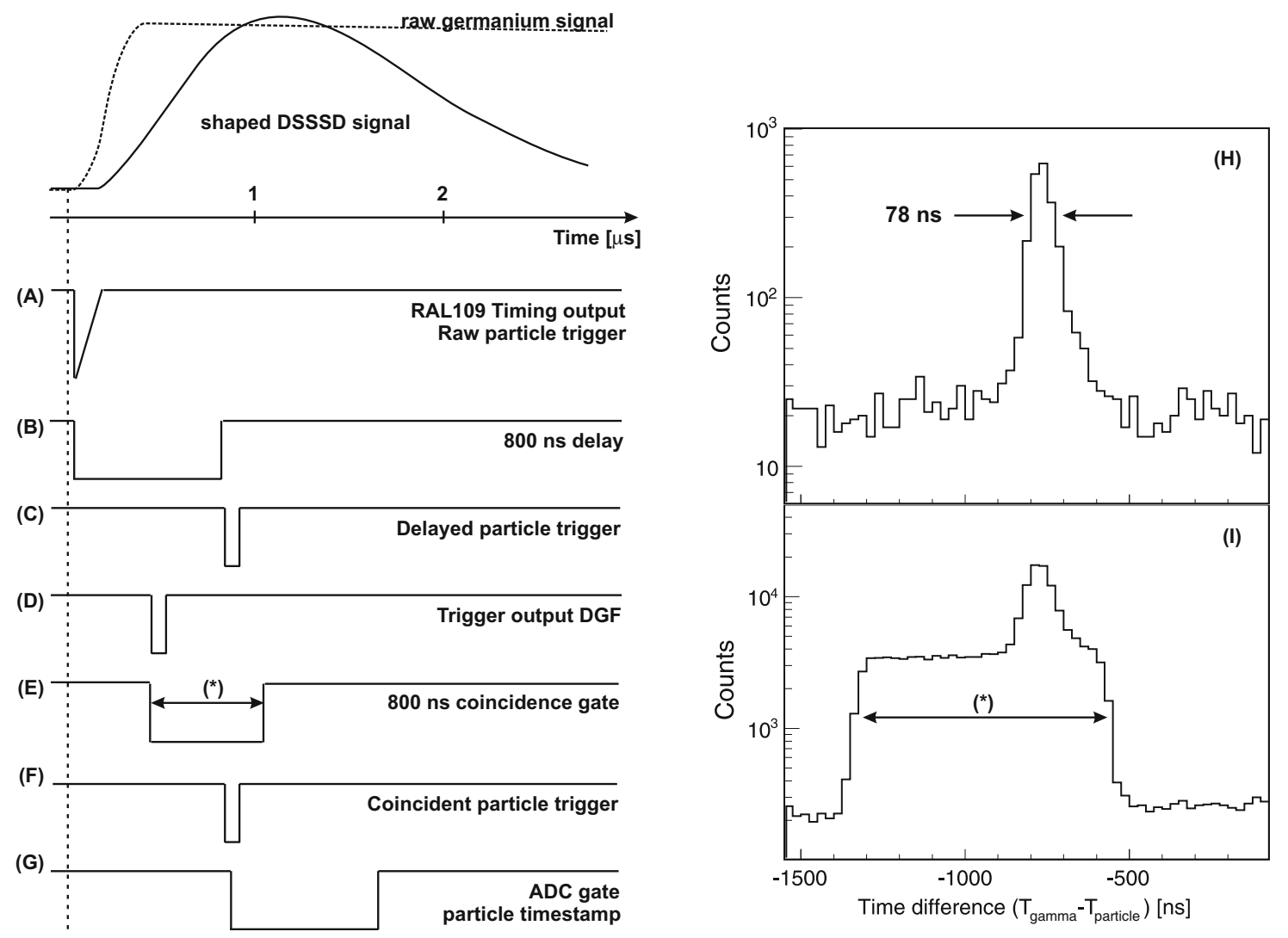

Fig. 16. The particle- $\gamma$ coincidence scheme for Coulomb excitation experiments. For transfer reaction experiments, the RAL109 energy and timing signals are replaced by the signals from the Mesytec electronics. (A) timing signal of the scattered particle, (B) gate generated to delay the particle trigger (A), (C) delayed particle trigger, (D) "OR" of timing outputs from all the DGF modules with signals from core electrodes, operated in leading edge mode, (E) each gamma ray in the full germanium array opens a coincidence window of $800 \mathrm{~ns},(\mathrm{~F})$ accepted particle trigger $(\mathrm{G})$ a gate of roughly $1 \mu$ s is generated with each accepted particle and is send to the ADC gate input and to the input of a DGF module to digitally timestamp the particle. (H) Resulting time difference spectrum between the gamma and particle time stamp when ALL particles are accepted (without the coincidence condition), (I) a similar spectrum from a different dataset, but with the coincidence condition applied and with the non-coincident particles (outside the region marked with a bracketed asterisk) downscaled by a factor of $2^{4}$.

${ }^{80} \mathrm{Zn}$ beam. Inspecting the difference between the "beamon" and "beam-off" spectra, a clear difference is observed up to $300 \mathrm{keV}$. This low-energy background radiation during the "beam-on" time windows stems from X-ray radiation emitted from the last accelerator cavity (9 gap resonator), which was located $\approx 5 \mathrm{~m}$ from the Miniball spectrometer. Furthermore, both spectra contain similar $\beta$-decay background radiation, whereas only the "beamon" spectrum contains radiation emitted during the reaction process (Coulomb excitation or transfer reaction).

\subsection{Time stamping}

Although it is possible for one DGF-4C to generate the $40 \mathrm{MHz}$ clock signal, used as timing reference, and to pass it in a daisy chain from one module to another, an external $40 \mathrm{MHz}$ clock was developed, which fans out the signal to all the DGF-4C modules. This results in greatly reduced clock jitter for the system, which has 54 DGF$4 \mathrm{C}$ modules [56]. Until 2009, scattered particles in the DSSSD were detected by analog VME electronics (CAEN
V785 ADC) and time information was provided by digital time stamping of these particles in dedicated DGF$4 \mathrm{C}$ CAMAC modules. The dead time for each detected event in one ADC was fixed to $10 \mu \mathrm{s}$. The CAEN V785 ADC's were replaced in 2009 by VME MADC modules from Mesytec [36], which provide additional internal time stamping, synchronized with an external clock, which was taken from the same $40 \mathrm{MHz}$ signal used by the DGF-4C modules. The main benefit of the new MADC modules is the shorter digitization time $(1.6 \mu \mathrm{s}$ instead of $6 \mu \mathrm{s})$. Timestamps for the start of the supercycle, the proton impact and the start of the "beam-on" window are still generated in dedicated DGF-4C modules. Offline gating on differences between the time stamps for particles, $\gamma$ rays and signals from the ISOLDE facility play a key role in improving the sensitivity of the Miniball array.

\subsection{Coincidence scheme}

Each $\gamma$-ray detected in Miniball generates a coincidence gate of $800 \mathrm{~ns}$ (see fig. 16(E)). Since the DGF modules generate a trigger output some $500 \mathrm{~ns}$ after the interaction of 
the $\gamma$-ray in the crystal (see fig. 16(D)), the particle trigger needs to be delayed by about the same amount in order to bring the particle trigger inside the $800 \mathrm{~ns}$ coincidence gate. The timing signal from the RAL109 amplifiers in the Coulomb-excitation setup, or the timing outputs from the Mesytec electronics in the transfer-reaction setup, are used to generate one "delayed particle" trigger (see fig. 16(A,B,C)). Each delayed particle trigger within the coincidence gate produces a "coincident particle" trigger (see fig. 16(F)). Either of these signals may be downscaled by a factor $2^{N}$, meaning that only one event in every $2^{N}$ is accepted and the logical OR of the downscaled signals is used to generate a gate on the corresponding ADC. For low-intensity beams (typically $<10^{4}$ ions/s) downscaling is not used and every particle generates a gate, unless it falls in the dead time of the ADC. For higher-intensity beams, the delayed particle trigger is downscaled but the coincident particle trigger is not. In this way, all particle$\gamma$ coincidences are acquired, but only 1 in $2^{N}$ particles without $\gamma$-rays are accepted. Note, however, that an event rejected by the downscaling of the delayed particle trigger, is still accepted if it is also a coincident particle. This downscaled mode is implemented to reduce the dead time in the particle-detection electronics due to elastically scattered particles. In fig. 16(right), the resulting time difference spectrum between a $\gamma$-ray and a particle is shown, without (top) and with (bottom) application of downscaling. In this example, the time resolution of the prompt peak was $\approx 78 \mathrm{~ns}$ (see fig. $16(\mathrm{H})$ ).

From the time spectra displayed in fig. 16 "prompt" ("random") spectra are generated by selecting the $\gamma$-rays in the prompt (random) time windows. An example of this procedure is shown in fig. 15, where the lower spectra are obtained with prompt (solid lines) and random (dashed) time cuts in the particle- $\gamma$ time difference spectrum. The background is reduced by a factor $10^{3}$. The Doppler-broadened transitions related to Coulomb excitation are clearly observed in the prompt spectrum. The limited amount of remaining background can be removed by subtracting the random spectrum. The resulting randomsubtracted spectrum in the region from 1200 to $1800 \mathrm{keV}$ is shown in fig. 29(top).

\subsection{Rate limitations}

The continuous beam released from the primary target (see fig. 12(B)), is bunched into a few REXEBIS pulses, as described in sect. 4.1. The beam intensity per REXEBIS pulse is thus much higher than the beam intensity per second from the primary target. If, for example, $5 \times 10^{5}$ ions $/ \mathrm{s}$ are extracted from the ion source at ISOLDE and the REXTRAP and REXEBIS operate at $10 \mathrm{~Hz}$ (cooling and breeding times of $100 \mathrm{~ms}$ ), allowing for $2 \%$ efficiency for trapping, charge breeding to the desired charge state and beam transport, $10^{3}$ particles are injected into the linac with each REXEBIS pulse. The particles arrive at the Miniball setup within $100 \mu \mathrm{s}$ ("standard" extraction). This is equivalent to an instantaneous rate of $10^{7}$ ions $/ \mathrm{s}$. A
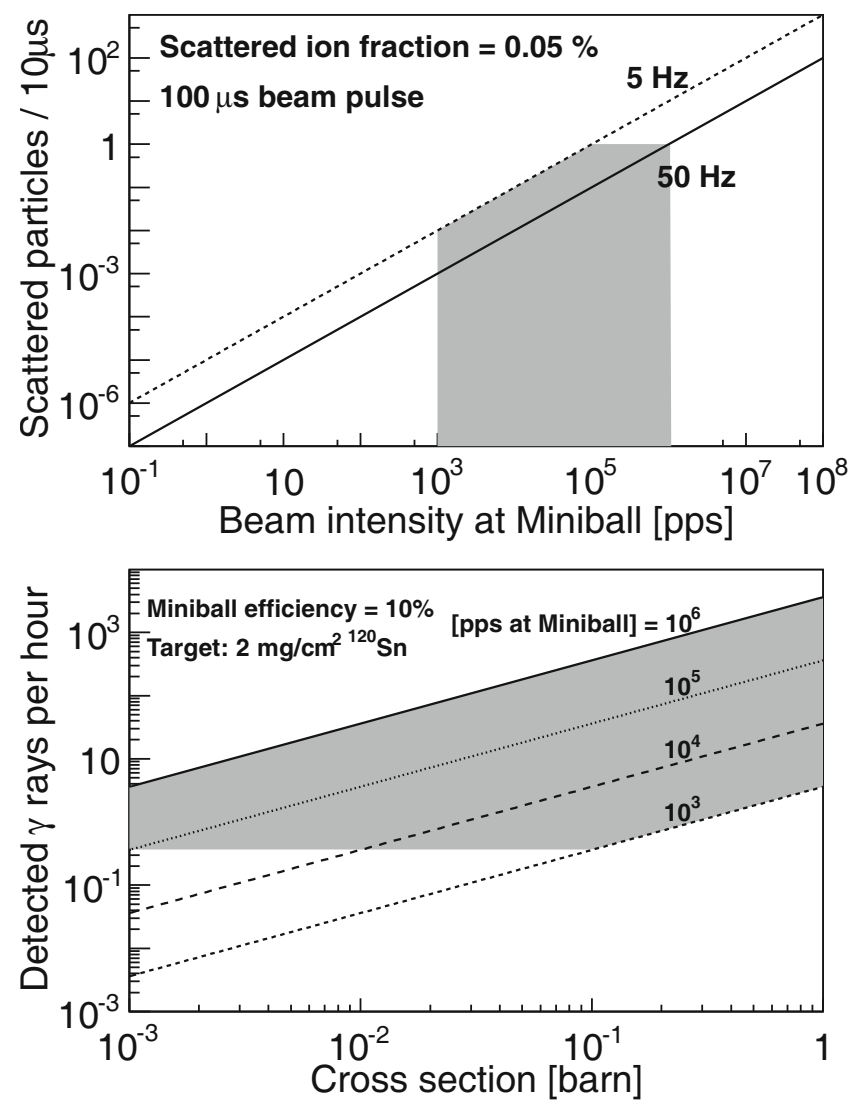

Fig. 17. (Top) An average of one scattered particle every $10 \mu \mathrm{s}$ can be considered as the limit to avoid considerable loss in detection efficiency, indicating the maximum beam intensity at Miniball which can be handled at the Miniball setup. (Bottom) Count rate per hour for a typical Coulomb-excitation experiment at REX-ISOLDE. The gray shaded areas indicate the regions where the Miniball setup has been operational, assuming realistic beam times of at most $\approx 7$ days.

limit on the incoming beam intensity is imposed by the dead time in the particle detector electronics, which corresponds to $10 \mu \mathrm{s}$ for the CAEN ADC's and $4.65 \mu \mathrm{s}$ for the MADCs. Figure 17(top) shows the maximum instantaneous beam intensities at Miniball, which can be handled without considerable loss in particle-detection efficiency. In this figure, typical REXTRAP and REXEBIS repetition rates for heavy and light ion beams are used of 5 and $50 \mathrm{~Hz}$, respectively. Furthermore, it is assumed that a typical fraction of $0.05 \%$ of the incident beam is scattered in the particle detectors. Figure 17(bottom) summarizes the achievable statistics per hour as a function of the cross section. For experiments with unusually high beam intensities, radiation damage to the particle detector imposes an additional limit, but this can be circumvented by shielding the innermost rings of the detector.

\section{Beam monitoring}

The weak RIBs with typical intensities of $10^{3}-10^{4}$ pps cannot be detected with standard Faraday Cups (FC's) and 
Micro Channel Plates (MCP's) in the linac. In order to optimize the transmission of the linac, an intense stable beam with similar $A / Q$ as the low-intensity radioactive beam (the "pilot" beam) is focused on the secondary target. A small scaling of the magnet and RF settings in the linac to the $A / Q$ of the radioactive ion beam ensures a similar focusing of the low-intensity RIB. In the original Miniball setup, only a gas-filled Parallel Plate Avalanche Counter (PPAC) [57] was used to monitor the beam profile $\approx 50 \mathrm{~cm}$ after the secondary target. However, it was soon recognized that a highly segmented detector on the target wheel is more suitable to focus the RIB, especially in cases where the $A / Q$ scaling from the pilot beam is relatively large. This only became possible with the newly designed "T-REX" target chamber, where a diamond detector could be positioned on the target ladder. In the original design of the target chamber (see fig. 7 which has been used since 2001), there was no possibility to insert an additional detector on the secondary target position. At the same time, an additional active collimator was inserted in front of the target chamber in order to optimize the transmission from the linac section to the Miniball target chamber. In the following, the PPAC, active collimators and diamond detector are described briefly.

\subsection{PPAC}

To monitor the beam position of the post accelerated radioactive ion beam, a Parallel Plate Avalanche Counter (PPAC) was positioned originally $50 \mathrm{~cm}$ behind the $\mathrm{sec}-$ ondary target and permanently inside the beampipe. In 2006, the gas counter was moved further downstream ( $\approx 1 \mathrm{~m}$ from the secondary target) and positioned on an actuator which allowed the gas counter to be removed from the beam line. The latter made it possible to position beam composition detectors after the PPAC and ensured a safer operation of the vacuum system.

The PPAC consists of $25 \mathrm{X}$ and $25 \mathrm{Y}$ wires with $\approx 1.6 \mathrm{~mm}$ pitch and is filled with 5 mbar of $\mathrm{CF}_{4}$. A complete description of the PPAC can be found in [57]. The instantaneous rate, which can amount up to $10^{10}$ particles per second prevents an event-by-event readout of the $\mathrm{X}$ and $\mathrm{Y}$ wires. Therefore, the detector cannot be used to tag $\gamma$-rays with individual particles which were not scattered at large angles. During operation at REX-ISOLDE, the wires of the PPAC are read out in "current" mode, by which the current from the wires is converted into a frequency. The recorded frequency per wire is used to provide an instantaneous picture of the beam spot, which can be used for beam tuning. The frequency per wire is accumulated over $1 \mathrm{~s}$ periods and a typical $1 \mathrm{~s}$ snapshot is shown in fig. 18.

\subsection{Active collimator}

The collimator, shown in fig. 19, consists of an aperture of $10 \mathrm{~mm}$, surrounded by four silicon PIN diodes of $1 \mathrm{~cm}^{2}$ active area. This collimator is positioned at the entrance of the Miniball target chamber. Special preamplifiers with

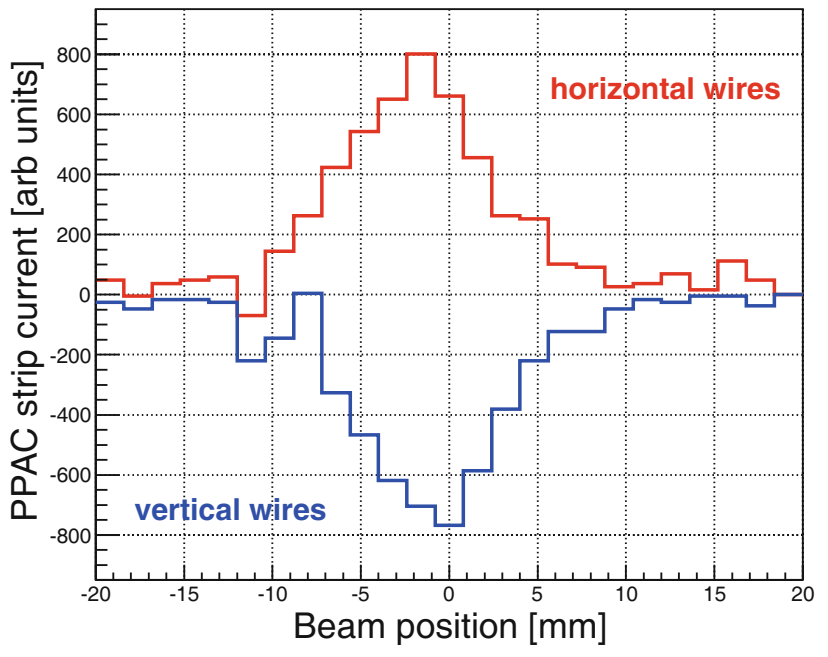

Fig. 18. (Color online) Snapshot of an integrated beam pulse from REX-ISOLDE, detected with the Parallel Plate Avalanche Counter, located $50 \mathrm{~cm}$ downstream from the secondary target.

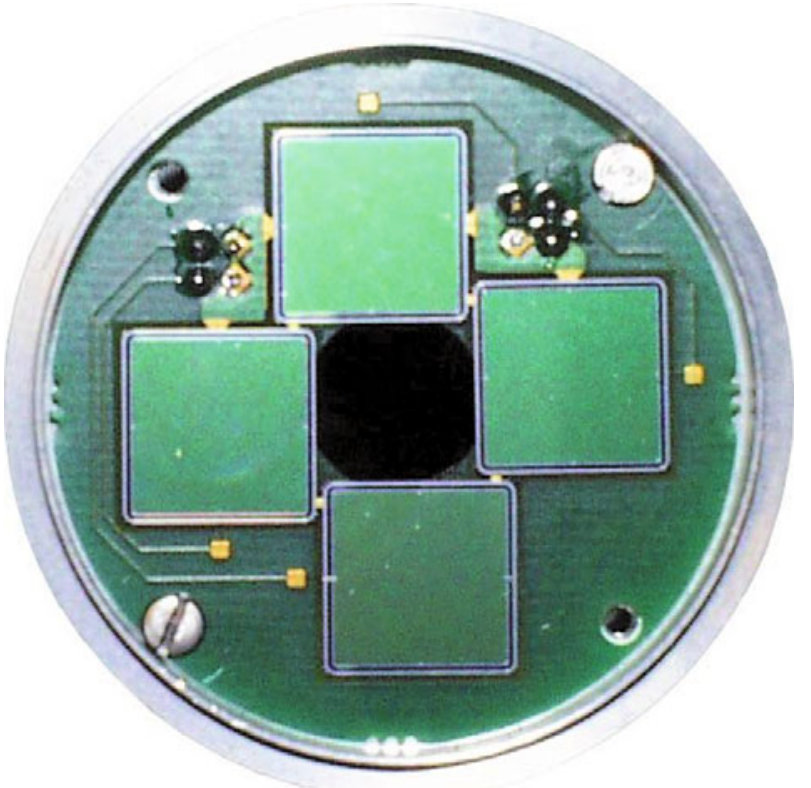

Fig. 19. (Color online) Active collimator consisting of four silicon PIN diodes of $1 \mathrm{~cm}^{2}$ active area mounted on a standard PCB board.

long integration times were developed in order to produce a pulse which is proportional to the total beam intensity on the PIN diodes. The preamplifiers start integrating the charge at the beginning of the "on-beam" window. The total integration time is slightly smaller than the "on-beam" time window. At the end of the integration time, the output pulses from the four diodes are fed into a standard VME ADC. A similar integration is done during the "offbeam" window (no incident beam), in order to obtain a proper offset calibration. This collimator aids in optimizing the transmission from the linear accelerator into the Miniball target chamber. 


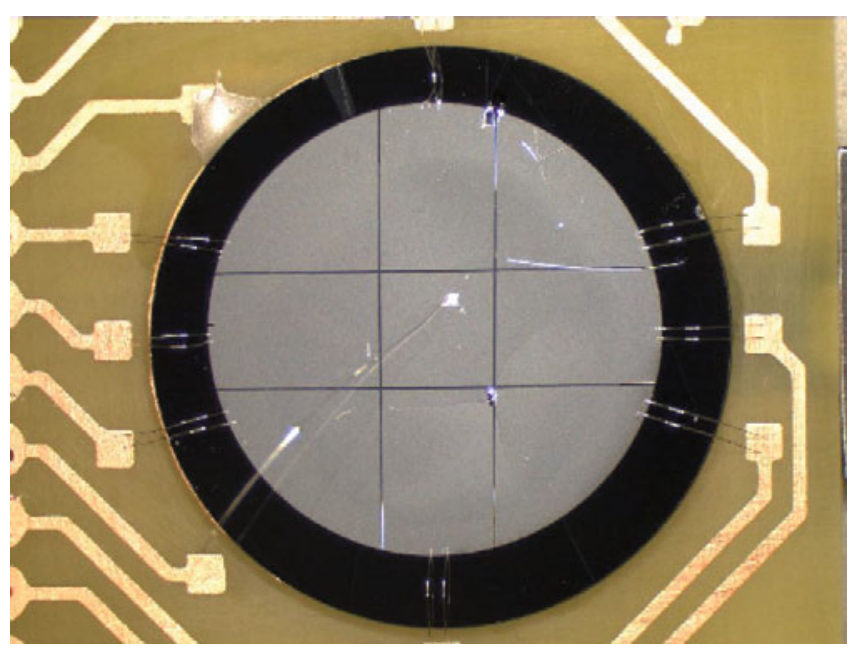

Fig. 20. (Color online) Photograph of the 9-fold segmented ultra-thin diamond beam detector.

\subsection{Target diamond detector}

A new type of ultra-thin, radiation-hard diamond detectors was developed to allow for a direct measure of beam profile on target. Due to its intrinsic properties, diamond is an ideal material for this type of detector [58]. Its large band gap and strong crystal binding make it radiation hard at room temperature. For the Miniball application, detectors with a maximum thickness of $10 \mu \mathrm{m}$ were produced, such that most of the available post-accelerated beams at REX-ISOLDE at $3 \mathrm{MeV} / A$ will loose only about $50 \%$ of their energy and $\approx 99 \%$ of the multiple-scattered particles still leave the target chamber through its exit tube. The latter is crucial in order to prevent a significant fraction of the radioactive ion beam from being deposited in the target chamber during beam tuning. The diamond detector is electrically segmented in 9 parts, as shown in fig. 20. This permits the focusing of the radioactive beam in the central region of $\approx 2.5 \times 2.5 \mathrm{~mm}^{2}$. The detector is installed on one of the six available positions of the target holder of the T-REX setup.

\section{Beam purity}

Beam contamination can originate from several sources:

- isobaric contaminants from the primary target which cannot be separated by the mass separator as they have the same mass;

- isotopes from the primary target with an integer multiple of both mass and charge;

- stable isotopes from the buffer gas in the REXTRAP (e.g., $\mathrm{Ne}, \mathrm{Ar})$;

- stable isotopes from the residual gas in the REXEBIS (e.g., C, O);

- stable isotopes from components of the REXEBIS (e.g., La from the cathode).

A measurement of the beam composition is crucial, since the post-accelerated beams can suffer from severe isobaric contamination, because the production rates for the RIB of interest is often orders of magnitude less than for other isotopes. In the case of Coulomb-excitation studies with Miniball, it is not the absolute transition rates for transitions in the projectile nucleus, which are measured, but rather these rates are determined relative to known transitions in the target nucleus. This is trivial for the case of a pure beam, but when beam contaminants are present, they also excite the target nucleus and this has to be taken into account by correcting for the fraction of the target excitation due to the projectile of interest. Consequently, the (relative) beam composition enters directly into the normalization of the experiment to the known Coulombexcitation cross section of the stable target isotope.

If a beam consisting of several projectiles $P_{i}$ bombards a target $T$, the unknown Coulomb excitation cross section $\sigma_{P_{i}}^{T}$ for the de-excitation of $P_{i}$ following excitation by $T$ is related to the known cross section $\sigma_{T}^{P_{i}}$ for the de-excitation of $T$ following excitation by $P_{i}$ by

$$
\sigma_{P_{i}}^{T}=\sigma_{T}^{P_{i}} \frac{N_{P_{i}}^{T}}{N_{T}^{P_{i}}},
$$

where $N_{P_{i}}^{T}$ is the number of events where $P_{i}$ de-excites after excitation by $T$ and $N_{T}^{P_{i}}$ is the number of events where $T$ de-excites after excitation by $P_{i}$. The former quantity is related directly to the efficiency-corrected integral of the peak corresponding to a $\gamma$-ray in the projectile, but the corresponding integral of a target-excitation peak gives not $N_{T}^{P_{i}}$ for a specific projectile but rather the sum over all projectiles in the beam, $N_{T}^{\text {total }}$.

The value for a specific projectile must be deduced from a knowledge of the composition of the beam,

$$
N_{T}^{P_{i}}=N_{T}^{\text {total }} \times\left[\frac{I_{P_{i}} \sigma_{T}^{P_{i}}}{\sum_{P_{j}} I_{P_{j}} \sigma_{T}^{P_{j}}}\right],
$$

where $I_{P_{j}} / I_{P_{i}}$ is the relative intensity of the projectiles $P_{j}$ and $P_{i}$ in the beam and the $\sigma_{T}^{P_{i}}$ can be calculated from the known target matrix elements for any projectile.

In this section, methods to determine the ratio $I_{P_{i}} / I_{P_{j}}$ and how this ratio can be optimized, in order to obtain a better signal to background ratio, are described.

\subsection{Bragg detector}

A first beam diagnostics detector is based on the concept of a Bragg ionization chamber. The detector is a cylindrical $7 \mathrm{~cm}$ long volume with a diameter of $5 \mathrm{~cm}$, containing 20 electrodes, geometrically isolated by $3 \mathrm{~mm}$ spacers and connected through a resistive chain to the high voltage. The electrodes are separated from the anode by a Frisch Grid, consisting of $50 \mu \mathrm{m}$ copper wires equally spaced at $0.5 \mathrm{~mm}$. The anode is located $1 \mathrm{~mm}$ behind the Frisch Grid and connected to a separate high voltage [59]. The measurement principle relies on the time evolution of the anode pulse, which corresponds to the energy loss curve of the incoming particle. The anode pulse is sampled by a 


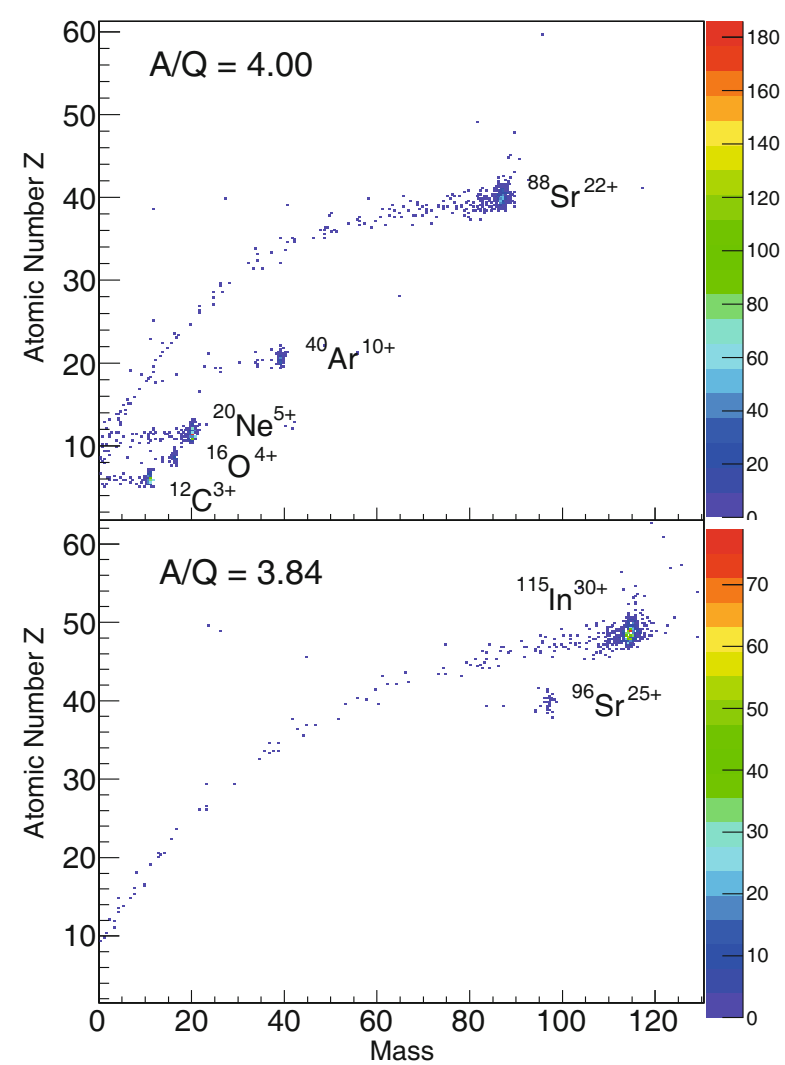

Fig. 21. (Color online) Spectra measured with the Bragg detector in the beam dump of the REX linac with (top) a standard $A / Q=4.00$ calibration beam, consisting of ${ }^{12} \mathrm{C},{ }^{16} \mathrm{O}$, ${ }^{20} \mathrm{Ne}$ and ${ }^{40} \mathrm{Ar}$ from the REXTRAP and REXEBIS residual gas and ${ }^{88} \mathrm{Sr}^{22+}$ from the ISOLDE mass marker. (Bottom) a $A=115$ radioactive ion beam from the ISOLDE primary target at $A / Q=3.84$.

SIS 3300 sampling ADC at a frequency of $100 \mathrm{MHz}$. Digital processing of these pulses yields the total range, energy and differential energy loss information. The traces are all written to disk and can be analyzed offline with optimized pulse shape analysis algorithms. The total beam needs to be attenuated several orders of magnitude in order to avoid pile-up and to reduce the data rate. Originally, this detector was mounted on a separate beam line, but it can now be mounted directly after Miniball, where a vacuum valve can be closed between the two during normal measurement and opened for measurements with the Bragg detector. The target wheel is rotated to an empty position for such measurements, though it is also possible to get a qualitative determination of changes in the beam composition with the secondary target in place.

Figure 21 shows the result of an analysis of the recorded traces from the Bragg chamber, obtained with different beams. The upper figure was measured with a standard calibration beam consisting of ${ }^{12} \mathrm{C},{ }^{16} \mathrm{O},{ }^{20} \mathrm{Ne}$ and ${ }^{40} \mathrm{Ar}$, which are residual-gas atoms from REXTRAP and REXEBIS, appearing at $A / Q=4.0$ after charge breeding. The heavier isotope, ${ }^{8}{ }^{8} \mathrm{Sr}$, is a stable mass marker coming from the ISOLDE ion source. The ${ }^{96} \mathrm{Sr}^{25+}$ and ${ }^{115} \mathrm{In}^{30+}$ beams were obtained from proton induced fis-
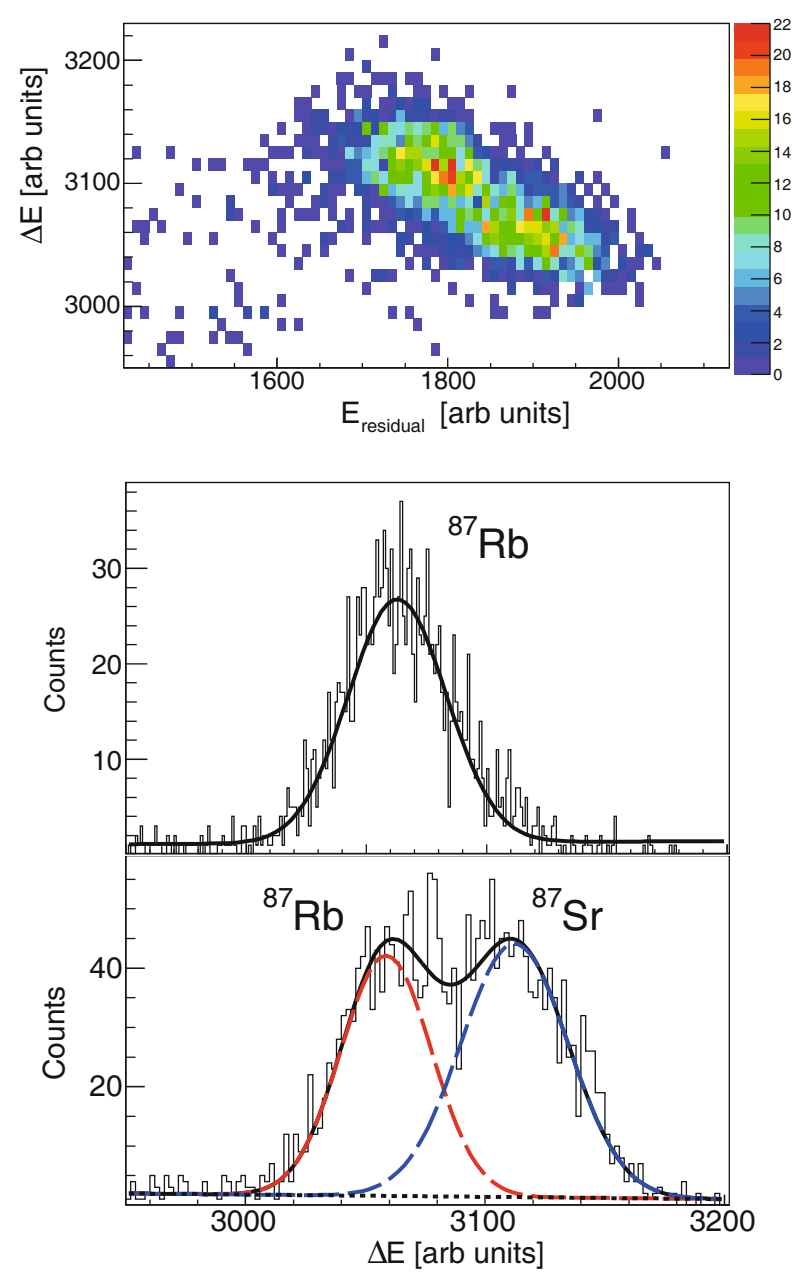

Fig. 22. (Color online) Top: $\Delta E-E$ spectrum measured with stable ${ }^{87} \mathrm{Rb}$ and ${ }^{87} \mathrm{Sr}$ beams. Middle: the projection onto the $\Delta E$ axis for a pure ${ }^{87} \mathrm{Rb}$ beam. Bottom: the same for the mixture of ${ }^{87} \mathrm{Rb}$ and ${ }^{87} \mathrm{Sr}$. These spectra illustrates the resolution of contributions of isobaric species up to $Z \approx 38$ and $A \approx 100$ with the $\Delta E-E$ telescope in the beam dump of the REX linac.

sion of a $\mathrm{UC}_{x}$ target [60]. More details on the production of the ${ }^{96} \mathrm{Sr}$ beam can be found in sect. 6.5. The calibration of the detector with known beams in the same mass region as the radioactive beam is crucial in order to identify all components in the weak radioactive beam.

Separation of adjacent isobars can only be achieved for elements up to $Z \approx 40$ though it has been possible to obtain a ratio between ${ }^{122} \mathrm{Cd}$ and a ${ }^{122} \mathrm{In}$ contaminant by fitting the peak as a doublet [59].

\section{$6.2 \Delta E-E_{\text {res }}$ detector}

A second detector, which is used routinely, is a relatively straightforward $\Delta E$ (gas)- $E(\mathrm{Si})$ telescope, consisting of a $\approx 2 \mathrm{~cm}$ gas volume $\left(\mathrm{CF}_{4}\right)$ with controlled pressure $(250$ 500 mbar) and a PIPS silicon detector. The instantaneous rate of the incident beam is reduced such that $\approx 5$ particles per REXEBIS pulse enter the gas detector. The entrance foil to the gas detector is a $\approx 100 \mu \mathrm{m}$ aluminized Mylar ${ }^{\circledR}$ foil. In fig. 22 (top), the measured $\Delta E$ (gas)- $E_{\text {residual }}(\mathrm{Si})$ 


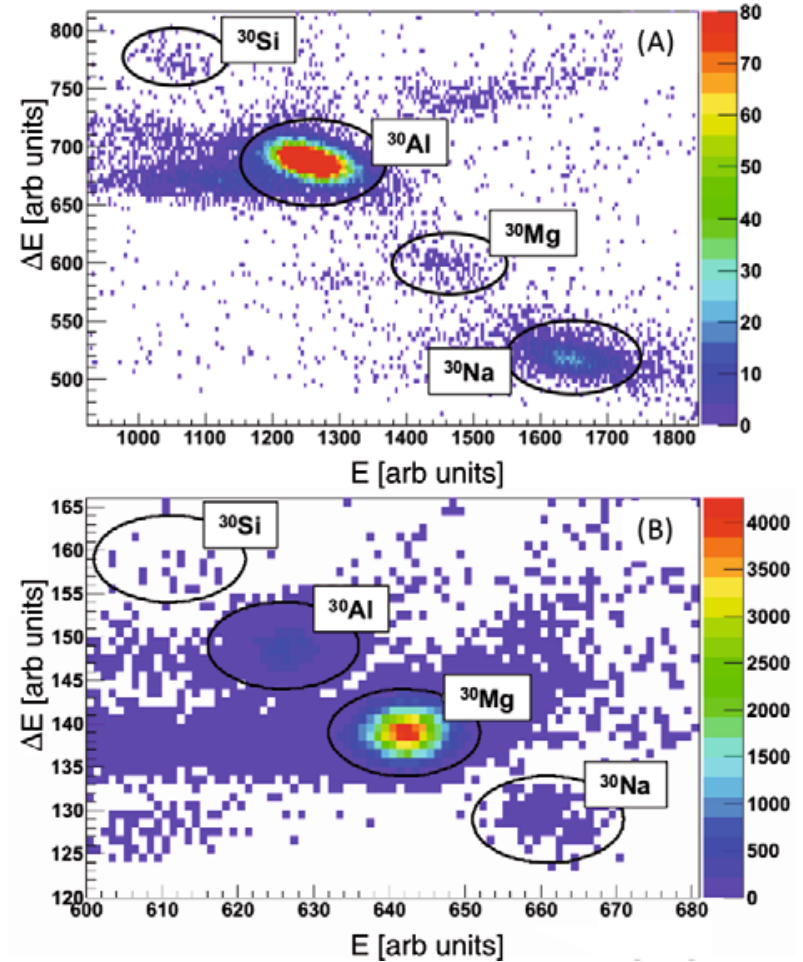

Fig. 23. (Color online) $\Delta E-E$ spectrum, measured with a radioactive $A=30$ beam, produced with a standard surface ionization ion source (A) and with a laser ion source (RILIS) tuned to resonance ionization of $\mathrm{Mg}(\mathrm{B})$. A huge enhancement of the laser ionized species is observed in (B).

spectrum is shown, obtained with a pure, stable ${ }^{87} \mathrm{Rb}$ reference beam from the ISOLDE target-ion source (top). The bottom spectrum of fig. 22 is measured with a $A=87$ beam, produced by proton induced fission of a $\mathrm{UC}_{x}$ target, equipped with a standard surface ion source. The additional component is identified as ${ }^{87} \mathrm{Sr}$. It is clear from these measurements that a separation of medium-heavy isobars with $\Delta Z=1$ is still possible up to $Z \approx 40$. The $\Delta E$ - $E$ telescope is used as an alternative to the Bragg detector, mounted either downstream of Miniball or on another beam line and can only be used without a secondary target in the beam. The primary advantage compared to the Bragg detector is its ease of use. Measurements with this detector are performed on a regular basis throughout an experiment to monitor the evolution of the beam composition.

\subsection{Selective laser ionization}

The Resonance Ionization Laser Ion Source (RILIS) is the most selective ion source at ISOLDE, since it relies on the resonant ionization of one specific element by means of up to three laser frequencies. A recent review on the RILIS system can be found in [61].

In fig. 23 two $\Delta E-E$ identification plots are shown for the radioactive ion beams of $A=30$, both produced with $1.4 \mathrm{GeV}$ protons on a $\mathrm{UC}_{x}$ target. In fig. $23(\mathrm{~A})$, a standard surface ion source was used and the beam is composed of
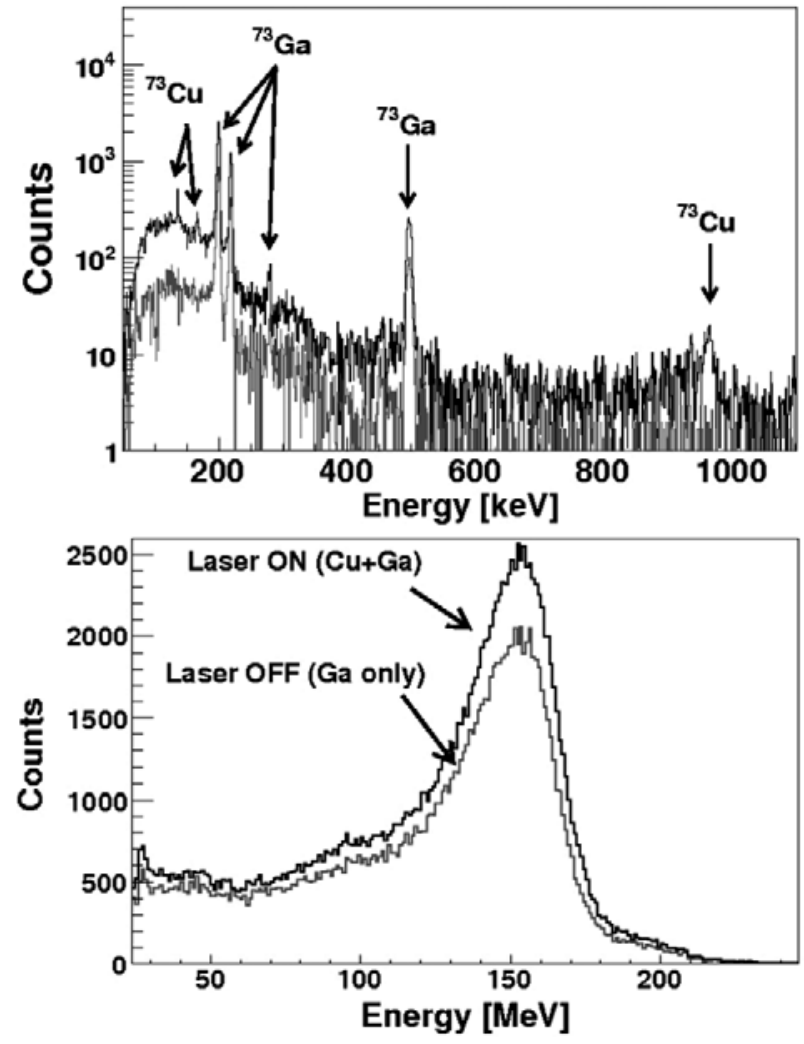

Fig. 24. (Top) Prompt $\gamma$-ray spectrum, random subtracted and Doppler corrected for scattered beam-like particles $\left({ }^{73} \mathrm{Cu}+{ }^{73} \mathrm{Ga}\right)$. The black (gray) spectrum was obtained during laser on (off) periods. (Bottom) Prompt particle spectrum, for all strips of the DSSSD, laser on (black) and laser off (gray).

${ }^{30} \mathrm{Al},{ }^{30} \mathrm{Na},{ }^{30} \mathrm{Mg}$ and ${ }^{30} \mathrm{Si}$, with decreasing intensity. This beam was used to perform a Coulomb excitation study of ${ }^{30} \mathrm{Na}$ [62]. In fig. 23(B), the beam composition is shown when the RILIS is used with a similar $\mathrm{UC}_{x}$ target and $1.4 \mathrm{GeV}$ proton beam and when the lasers are tuned to ionize $\mathrm{Mg}$. It is clear that the $\mathrm{Mg}$ contribution to the $A=30$ beam is orders of magnitude higher with RILIS. In order to deduce the ratio of laser ionized species to isobaric contaminants, the laser ionization is switched on and off periodically, with a repetition rate equal to and synchronized with the CERN supercycle (see fig. 12(A)). The normalization of Coulomb excitation experiments can be achieved by comparing either the amount of target excitation or by comparing the number of scattered particles in the DSSSD during laser on and laser off periods. An extensive description of both methods can be found in [23].

An example of laser on and laser off spectra (prompt and Doppler corrected for the scattered beam-like particles) is given in fig. 24, which shows the Coulomb excitation spectrum obtained with a $2.9 \mathrm{MeV} / A$, laser ionized, radioactive ${ }^{73} \mathrm{Cu}$ beam [63]. The prompt $\gamma$-rays in the laser off spectra are related to the surface ionized ${ }^{73} \mathrm{Ga}$ isobaric contaminants in the beam. The particle spectrum in the bottom of fig. 24 is the summed spectrum from one DSSSD, measured during laser on and laser off periods. From the difference in counts in this spectrum, the ratio $I_{C u} / I_{G a}$ can be deduced. 


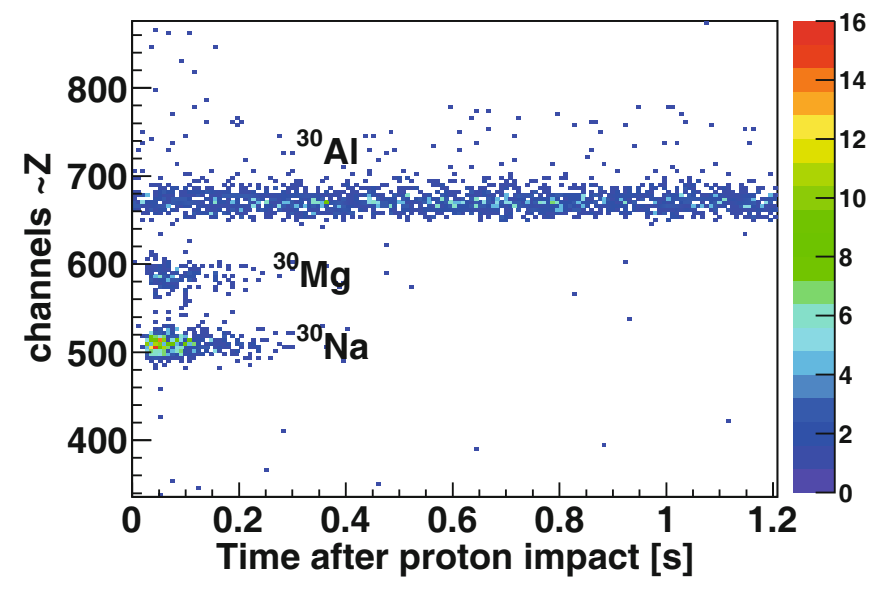

Fig. 25. (Color online) Energy loss in the gas detector (proportional to the atomic number $Z$ ) versus the detection time in the $\Delta E-E$ telescope in the beam dump after the proton impact. The upper particles are ${ }^{30} \mathrm{Al}$, with a half-life of $3.6 \mathrm{~s}$, the middle particles are ${ }^{30} \mathrm{Mg}\left(T_{1 / 2}=335 \mathrm{~ms}\right)$ and the lower particles are ${ }^{30} \mathrm{Na}\left(T_{1 / 2}=48 \mathrm{~ms}\right)$.

The amount of laser ionized isotopes in the radioactive beam can also be monitored during the experiment by following the characteristic $\gamma$ decay of the laser ionized radioactive isotope in the Miniball spectrometer and in a germanium detector placed at the beam dump. An extensive description of this method is given in [64]. In some cases, physics results are obtained for the -in principleunwanted isobaric contaminants $[65,66]$.

\subsection{Release characteristics}

The release time of the produced radioactive isotopes from the thick primary target container depends on the diffusion and effusion characteristics of the chemical element. This, in turn, depends on a variety of parameters, such as target and surface materials, temperature, etc. A review on this topic can be found in [67]. In this section, it will be shown that a weak radioactive ion beam, which is overwhelmed by (stable) contaminants from the primary target, can be purified as long as the release time for different isotopes differs considerably. A first example is given by $\mathrm{Ni}$ isotopes, which diffuse very slowly out of the primary target with time constants up to hours. Evidently, short lived $\mathrm{Ni}$ isotopes cannot be extracted from thick primary targets at ISOLDE in sufficient quantities to perform reaction studies. Nevertheless, ${ }^{68} \mathrm{Ni}\left(T_{1 / 2}=19(3) \mathrm{s}\right)$ can still be extracted with an average rate of $8 \times 10^{5}$ ions per second using RILIS, a $\mathrm{UC}_{x}$ target and $2 \mu \mathrm{A}$ proton beam [68]. However, this $A=68$ beam is heavily contaminated by ${ }^{68} \mathrm{Ga}$, which is released much faster than ${ }^{68} \mathrm{Ni}$. In [69], a description is given how a highly purified ${ }^{68} \mathrm{Ni}$ beam can be obtained. The rationale behind the method is to maximize the production of ${ }^{68} \mathrm{Ga}$ and ${ }^{68} \mathrm{Ni}$ with an intense sequence of consecutive proton pulses. Roughly $5 \mathrm{~s}$ after the proton pulses have been switched off, the ${ }^{68} \mathrm{Ga}$ intensity is reduced considerably and a relatively pure ${ }^{68} \mathrm{Ni}$ beam emerges from the target (see fig. 1 in [69]). Another example is given in fig. 25, where the gas signal from the $\Delta E-E$ telescope is plotted against time after the proton impact. As was already shown in fig. $23(\mathrm{~A})$, the ${ }^{30} \mathrm{Na}$ beam is heavily contaminated by ${ }^{30} \mathrm{Al}$ and, as can be seen from fig. 25 , the release time of ${ }^{30} \mathrm{Na}$ is much shorter than the release time of the much longer lived ${ }^{30} \mathrm{Al}$. By gating detected $\gamma$-rays on their arrival time after the proton impact, the prompt $\gamma$-spectrum can be purified considerably. Numerous other isotopes $(\mathrm{Zn}, \mathrm{Pb}, \mathrm{Po})$ can be identified, where the contaminant ( $\mathrm{Ga}, \mathrm{Fr}$ ) has a different release characteristics, such that the contamination level can be reduced by imposing stringent time conditions.

\subsection{Molecular extraction}

In some cases, the isobaric contamination (stable or radioactive) from the primary target is several orders of magnitude higher than the radioactive isotope of interest. One way to overcome this serious problem is to utilize molecular extraction from the primary target. This is possible when the chemical element of interest easily forms molecules with available "carrier" atoms. The isotope of interest is mass separated at a higher mass value, where the isobaric contamination is minimal or non existing. Two examples are the molecular extraction of ${ }^{70} \mathrm{SeCO}$ $(A=98)[70]$ and ${ }^{96} \mathrm{SrF}^{+}(A=115)$ [71]. These beams were extracted from a $\mathrm{ZrO}_{2}$ target equipped with a MK5 hot plasma ion source and a $\mathrm{UC}_{x}$ target equipped with a MK8 hot plasma ion source, respectively. In both cases, additional gas leaks of $\mathrm{CO}$ and $\mathrm{CF}_{4}$, respectively, were input to the primary target container. The molecules are trapped and cooled in the REXTRAP, where the mass of the molecule is used for the applied cooling frequency. The break-up of the molecules happens primarily in the REXEBIS ion source. The $A / Q$ separator after the REXEBIS selects the highly purified beam of radioactive isotopes. The overall efficiency of the REXTRAP and REXEBIS combination of this production method is $2-5 \%$ [72]. Figure 21(bottom) shows the beam composition, measured with the Bragg detector in the beam dump after molecular extraction of the ${ }^{96} \mathrm{SrF}$ beam and break-up of the molecule in the REXEBIS. The remaining ${ }^{115} \mathrm{In}^{30+}$ component in the beam stems from the initial mass of the $\mathrm{SrF}$ molecule, which is $A=115$. The actual Coulomb excitation experiment was performed at an $A / Q=4.17$ setting, where the ${ }^{115}$ In contamination is not present. Beams of ${ }^{140,142} \mathrm{BaF}^{+}(A=159,161)$ were obtained in a similar manner [73].

\subsection{Stripper foil}

In one experiment to study ${ }^{17} \mathrm{~F}$, it was possible to eliminate the contamination from ${ }^{17} \mathrm{O}$ completely by inserting a stripper foil between the REX accelerator and the bending magnet before Miniball. By selecting the $9+$ (i.e. fully stripped) charge state with the bending magnet, it was possible to obtain a pure fluorine beam. 


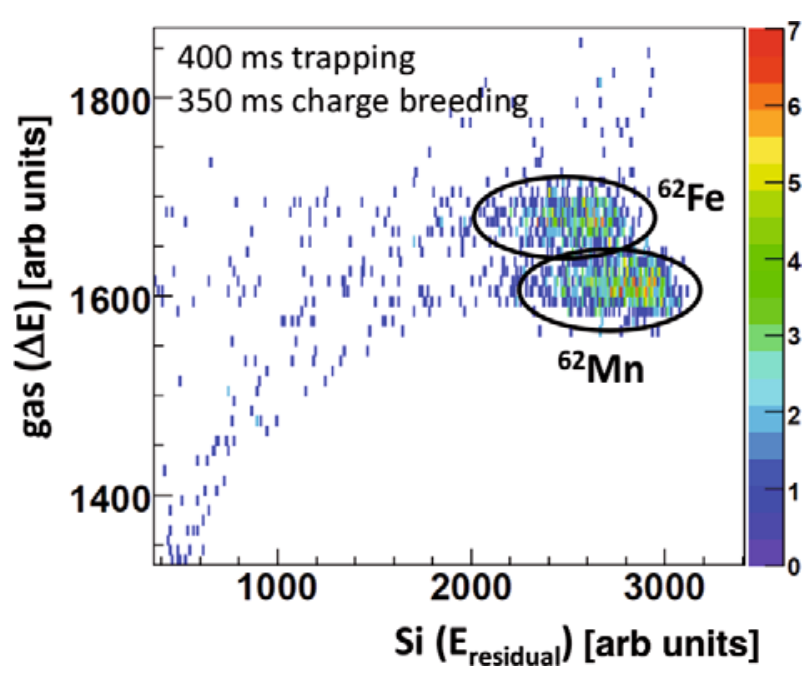

Fig. 26. (Color online) Beam composition measured in the $\Delta E-E_{\text {res }}$ detector of the ${ }^{62} \mathrm{Mn}$ beam when the produced isotopes are trapped for $400 \mathrm{~ms}$ in REXTRAP and charge bred for $350 \mathrm{~ms}$ in the REXEBIS. The final post-accelerated beam is composed of ${ }^{62} \mathrm{Fe}$ and ${ }^{62} \mathrm{Mn}$, showing the feasibility to produce post-accelerated Fe beams.

\subsection{In-trap decay}

The diffusion and/or effusion times of some chemical elements, the so-called "refractory" elements, are much longer than the half-life of the radioactive isotopes, where potentially interesting nuclear structure information can be obtained. Examples are the neutron-rich $\mathrm{Ca}, \mathrm{Ti}, \mathrm{Cr}, \mathrm{Fe}$ and $\mathrm{Ni}$ isotopes. These short-lived isotopes cannot be extracted from the thick primary targets used at ISOLDE. In the region around $Z=28$, neutron-rich $\mathrm{Mn}$ isotopes are released fast and very selectively from $\mathrm{UC}_{x}$ targets, thanks to the highly efficient laser ionization scheme for Mn. These isotopes are at the same time short lived with half-lives less than $\approx 700 \mathrm{~ms}$ for $A>60$. Since the trapping and charge-breeding times in the REXTRAP and REXEBIS combination can be extended to the same order of magnitude as these half-lives, one can extract the mother $(\mathrm{Mn})$ isotopes together with the decay daughter (Fe) products from the REXEBIS. A first proof of principle has been obtained with an intense and pure beam of ${ }^{61} \mathrm{Mn}\left(T_{1 / 2}=670(40) \mathrm{ms}\right)$, which resulted in the Coulomb excitation of both ${ }^{61} \mathrm{Fe}$ and ${ }^{61} \mathrm{Mn}[22]$. A second experiment focused on the post-acceleration of both ${ }^{62} \mathrm{Mn}$ and ${ }^{62} \mathrm{Fe}$ isotopes. From the $\Delta E-E$ telescope, clear evidence was found that both isotopes are present in the beam (see fig. 26) when trapping and charge-breeding times of $400 \mathrm{~ms}$ and $350 \mathrm{~ms}$, respectively, were utilized. This in-trap decay and subsequent post-acceleration of mother and daughter isotopes requires that the REXTRAP and REXEBIS be synchronized with the proton impact, which is, in "standard" operation, not the case [74].

\section{Doppler correction}

Most of the nuclear energy levels which are excited by Coulomb excitation decay via fast (femto or pico seconds) transitions (in cascade) to the ground state. As a consequence, the excited nuclei de-excite in flight and the $\gamma$-ray energies are observed with an angle-dependent Doppler shift. If the angle $\vartheta_{\gamma}$ between the particle and the $\gamma$-ray and the speed of the particle are known, it is possible to correct for this Doppler shift on an event-by-event basis. The Doppler-corrected energy $E_{D C}$ is then given as a function of the energy measured in the laboratory frame $E_{L a b}$ by

$$
E_{D C}=\gamma E_{L a b}\left[1-\beta \cos \left(\vartheta_{\gamma}\right)\right],
$$

where $\gamma=1 / \sqrt{1-\beta^{2}}, \beta=v / c$ and $\vartheta_{\gamma}$ is related to the angles of the $\gamma$-ray $\left(\theta_{\gamma}, \phi_{\gamma}\right)$ and of the particle emitting the $\gamma$-ray $\left(\theta_{p}, \phi_{p}\right)$ by

$$
\cos \left(\vartheta_{\gamma}\right)=\sin \left(\theta_{p}\right) \sin \left(\theta_{\gamma}\right) \cos \left(\phi_{p}-\phi_{\gamma}\right)+\cos \left(\theta_{p}\right) \cos \left(\theta_{\gamma}\right)
$$

Thus, three key pieces of information are needed to correct the energy: the angle of the $\gamma$-ray and the angle and speed of the particle, which emitting the $\gamma$-ray. The precision, with which the angle of the particle may be determined, depends on the spatial resolution of the DSSSD. If the particle which emitted the $\gamma$-ray was the same as the one detected, the required angles are determined directly. If, however, this is not the case, the angles can be deduced from the angle of the detected particle using the kinematic relations. The value of $\beta$ is determined from the measured energy of the scattered particle in the DSSSD in case of Coulomb excitation. For transfer reactions $((\mathrm{d}, \mathrm{p})$ or $(\mathrm{t}, \mathrm{p})$ reactions $)$, the scattering angle of the heavy beam-like projectile is deduced from the detected angular coordinates of the detected recoiling proton. In principle, it is necessary to determine the angle of the $\gamma$ ray from the position of the first interaction, but as it is not possible to determine the order of the interactions with Miniball, the position of the main interaction is used instead. In add-back mode, where contributions from more than one detector in a cluster are combined, the Doppler correction is performed with the angular coordinates of the $\gamma$-ray with the highest energy.

To obtain the $\gamma$-ray intensity in the rest frame of the $\gamma$-emitting nuclei from the measured laboratory intensity, a correction has to be applied for the difference in solid angle, given by

$$
\frac{\mathrm{d} \Omega_{\text {rest }}}{\mathrm{d} \Omega_{l a b}}=\left(\frac{E_{\gamma}}{E_{\gamma 0}}\right)^{2}=\frac{1-\beta^{2}}{\left(1-\beta \cos \vartheta_{\gamma}\right)^{2}} .
$$

As the relation between the solid angles is given by the square of the Doppler shift, the $\gamma$-ray intensity distribution is peaked at forward angles, as shown in fig. 27 for different beam energies $(\beta=0.5$ and 0.07$)$. At REXISOLDE with typical velocities of $\beta \approx 0.07$, the effect of the Lorentz boost in forward direction is small, and the clusters can be configured in $4 \pi$ geometry with negligible loss in count rate. On the other hand, at relativistic energies, this effect becomes important and the forward positioning of the cluster detectors becomes relevant. One example is given in sect. 9.1, where the use of Miniball in the RISING setup at GSI is described. 


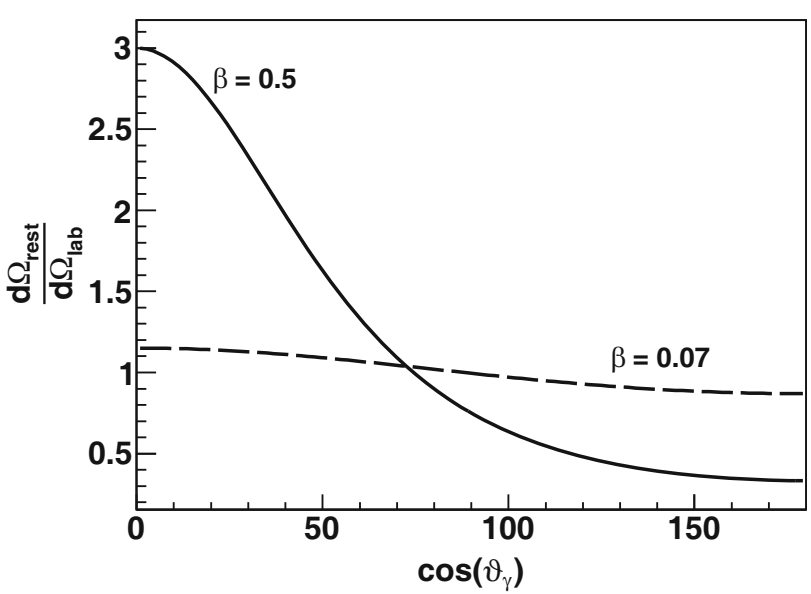

Fig. 27. Illustration of eq. (6) with $\beta=0.5$ and 0.07 . The latter value is the typical velocity at REX-ISOLDE.

The positions of the individual segments in the laboratory frame $\left(\theta_{\gamma}\right.$ and $\phi_{\gamma}$ in eq. (5)) are determined with the reactions $\mathrm{d}\left({ }^{22} \mathrm{Ne},{ }^{23} \mathrm{Ne}\right) \mathrm{p}$ and/or $\mathrm{d}\left({ }^{22} \mathrm{Ne},{ }^{23} \mathrm{Na}\right) \mathrm{n} .{ }^{22} \mathrm{Ne}$ is a typical residual gas in the REXTRAP+REXEBIS and an intense beam is available without involving the ISOLDE primary target. For this reaction a $10 \mu \mathrm{m}$ deuterated polyethylene ( $\mathrm{dPE})$ target is used. In the unconditioned singles spectrum, an intense Doppler-broadened $\gamma$-ray at $440 \mathrm{keV}$ is observed, corresponding to the $5 / 2^{+} \rightarrow 3 / 2_{\text {g.s }}^{+}$ transition in ${ }^{23} \mathrm{Na}$ and another at $1017 \mathrm{keV}$, corresponding to the $1 / 2^{+} \rightarrow 5 / 2_{\text {g.s }}^{+}$transition in ${ }^{23} \mathrm{Ne}$. Due to the kinematics of these reactions, the $A=23$ nuclei are barely deviated from $0^{\circ}$ and cannot be detected. However, in the case of the $\mathrm{d}\left({ }^{22} \mathrm{Ne},{ }^{23} \mathrm{Ne}\right) \mathrm{p}$ reaction, it is possible to gate on the protons and reconstruct the trajectory of the ${ }^{23} \mathrm{Ne}$ from the kinematics. However, this requires particle- $\gamma$ coincidences, so high statistics are needed and since there is very little angular straggling for the $A=23$ nuclei, the Doppler shift is, in any case, dominated by the angle of the $\gamma$-ray. So in general, the positioning is done solely with singles information and the inverse $(d, n)$ reaction, which has the advantage of a greater $\gamma$-ray efficiency at $440 \mathrm{keV}$ compared to that at $1017 \mathrm{keV}$.

In fig. 28 the solid line shows the uncorrected spectrum around $440 \mathrm{keV}$ and Doppler-corrected spectra using the different ways of determining the $\gamma$-ray angle: at cluster level, detector level or segment level. The average $\beta$ value after passage through $10 \mu \mathrm{m} \mathrm{dPE}$ is used and the ${ }^{23} \mathrm{Na}$ particles are assumed to recoil at $0^{\circ}$. The improvement from cluster level $(\mathrm{FWHM}=15 \mathrm{keV})$ to segment level $(\mathrm{FWHM}=5 \mathrm{keV})$ can be seen. This latter value contains contributions from the intrinsic resolution of the $\gamma$-ray detectors in the array, the precision of the determination of the $\gamma$-ray, the angular straggling of the particles, which causes deviations from $\theta_{p}=\phi_{p}=0^{\circ}$ and their energy straggling, which causes deviations around the average value of $\beta$.

A good peak-to-background ratio can be achieved by applying the background suppression techniques, described in sect. 4 and the Doppler correction described in this section. This is illustrated in fig. 29(top), where the

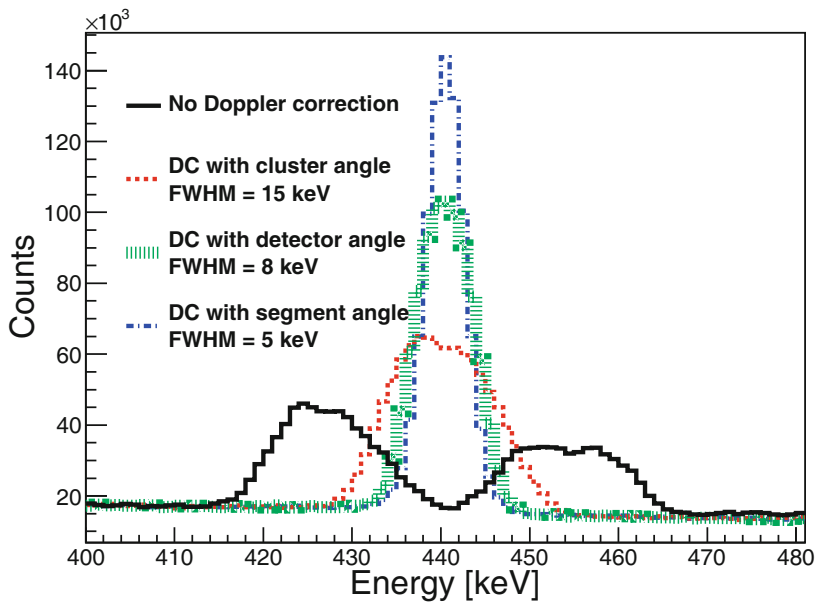

Fig. 28. (Color online) Singles spectra obtained during a $\mathrm{d}\left({ }^{22} \mathrm{Ne},{ }^{23} \mathrm{Na}\right) \mathrm{n}$ measurement. The $5 / 2^{+} \rightarrow 3 / 2_{\text {g.s. }}^{+}$transition at $440 \mathrm{keV}$ in ${ }^{23} \mathrm{Na}$ is observed showing a large Doppler shift in the singles spectrum (solid line). The double-hump structure is due to Miniball clusters being placed in two groups at forward and backward angles. Performing a Doppler correction using the position of each cluster results in a FWHM of about $15 \mathrm{keV}$ (dots). This is improved to $8 \mathrm{keV}$ by using the position of each detector capsule (dashes), while using the segment positions results in $5 \mathrm{keV}$ (dash-dot). The angle of the particle emitting the $\gamma$-ray was not considered as they are scattered to angles not covered by the particle detector.

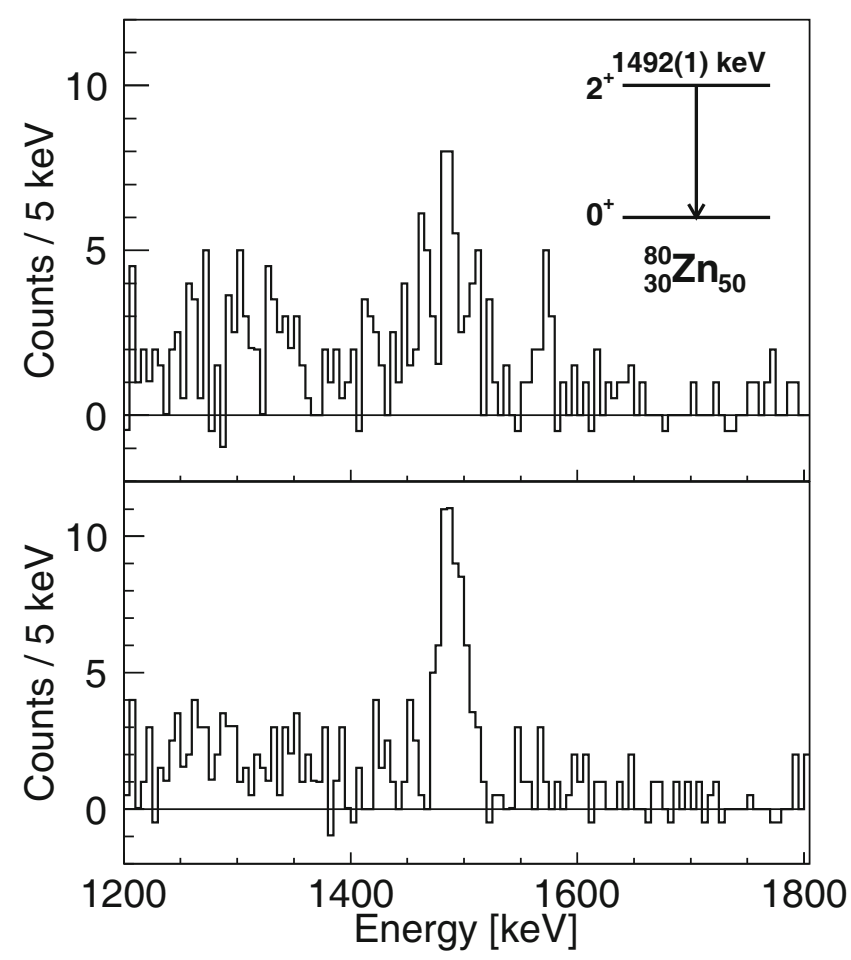

Fig. 29. (Top) Example of a prompt, random-subtracted and not Doppler-corrected Coulomb-excitation spectrum for ${ }^{80} \mathrm{Zn}$ on ${ }^{108} \mathrm{Pd}$ at $2.9 \mathrm{MeV} / A$ [75]. (Bottom) The same, but Doppler corrected at segment level. 
prompt spectrum, which was already shown in fig. 15 is repeated, but where the gray random spectrum of fig. 15 is now subtracted from the prompt spectrum. Additionally, the $\gamma$-spectra in fig. 29 are gated on detected beam-like particles in the DSSSD. In fig. 29(bottom), an additional Doppler correction at the segment level is applied, utilizing the angular coordinates of the detected beam-like particle. The peak-to-background ratio in the final DC spectrum is roughly 4 .

Although it has been shown [17] that PSA significantly improves the resolution for the $\mathrm{d}\left({ }^{37} \mathrm{Cl},{ }^{38} \mathrm{Ar}\right) \mathrm{n}$ and $\mathrm{d}\left({ }^{37} \mathrm{Cl},{ }^{38} \mathrm{Cl}\right) \mathrm{p}$ reactions at $1.9 \mathrm{MeV} / A$, it can be seen from fig. 5 that for Coulomb-excitation experiments at $\approx 2.9 \mathrm{MeV} / A$ the uncertainty in the particle direction and energy due to straggling in the target dominate and no significant improvement in the peak-to-background ratio is obtained when applying Doppler correction with angular coordinates of the $\gamma$-rays, determined from the onboard PSA. Nevertheless, Doppler correction at segment level still yields a crucial improvement in the peak-tobackground ratio.

\section{Selected results from REX-ISOLDE}

In this section some of the physics results from the Miniball campaigns at REX-ISOLDE are presented. Two distinct research topics can be distinguished:

- the changing shell structure far off the valley of beta stability probed by Coulomb excitation and transfer reactions;

- the investigation of shape co-existence by means of (multiple) Coulomb excitation and transfer reactions.

A more detailed overview of the physics results obtained at REX-ISOLDE can be found in [3]. The purpose of this section is to provide a brief overview of some of the highlight experiments, which illustrate the uniqueness of the REX-ISOLDE and Miniball combination. In table 3 all the isotopes which have been post-accelerated by REXISOLDE to Miniball in the period 2001-2012 are listed, together with the type of reaction that was induced and references to the published results.

\subsection{Evolution of shell structure}

\subsubsection{The "island of inversion" around ${ }^{30} \mathrm{Mg}$}

The physics case of the island of inversion around $Z=12$ and $N=20$ is described in numerous publications (refer to [97] for the original article introducing the name "island of inversion") and reviews [98]. One of the first successful experiments performed with the Miniball setup at REX-ISOLDE in this region was the low-energy Coulomb excitation of ${ }^{30,32} \mathrm{Mg}[27,77]$, which settled the issue of inconsistent $B(E 2)$ values previously measured in this region by means of intermediate energy Coulomb excitation.
In the following years, Coulomb excitation of ${ }^{31} \mathrm{Mg}[79]$ and ${ }^{29,30} \mathrm{Na}$ was performed with beam energies around $3 \mathrm{MeV} / A$. In previous sections, these experiments were used to illustrate the selective power of the RILIS ion source (see fig. 23(B)) and how to exploit the release characteristics to purify the Coulomb-excitation spectra. The series of these experiments has contributed to the determination of transition strengths and the discovery of excited states, used to test the predictions of shell-model calculations [99]. Comparison of the transition probabilities of $30,31,32 \mathrm{Mg}$ establishes that, for the $N=19$ magnesium isotopes, not only the ground state, but also excited states are largely dominated by a deformed pf intruder configuration.

In 2008, the first transfer reactions based on inverse kinematics were started in this region, with $\mathrm{d}\left({ }^{30} \mathrm{Mg}\right.$, $\left.{ }^{31} \mathrm{Mg}\right) \mathrm{p}$ as the first pioneering experiment and $\mathrm{t}\left({ }^{30} \mathrm{Mg}\right.$, ${ }^{32} \mathrm{Mg}$ )p as the first experiment utilizing both a radioactive beam and a radioactive secondary target. The latter experiment observed the shape-coexisting excited $0_{2}^{+}$ state in ${ }^{32} \mathrm{Mg}$ [44] using a ${ }^{30} \mathrm{Mg}$ beam provided by REXISOLDE on a tritium-loaded titanium foil and the T-REX setup. The measured angular distributions clearly identify the newly observed state as $0^{+}$. The state is crucial for the understanding of shape coexistence in this mass region.

\subsubsection{The region around ${ }^{68-78} \mathrm{Ni}$}

The shell closures at $Z=28$ and $N=40,50$ have been the subject of extensive experimental and theoretical investigations. It is anticipated that these shell closures are modified when filling neutrons in the $\nu \mathrm{g}_{9 / 2}$ or higher-lying orbitals or due to, e.g., the tensor part of the residual interaction or the enhanced neutron pair scattering from the pf shell to the $g_{9 / 2}$ unique parity intruder orbital. Following the work of Franchoo et al. [100] and Van Roosbroeck et al. [101] on neutron-rich $\mathrm{Cu}$ isotopes, which resulted in the discovery of the monopole migration of the $5 / 2^{-}$state in $\mathrm{Cu}$ isotopes beyond $N=40$ and three beta-decaying isomers in the $N=40$ nucleus ${ }^{70} \mathrm{Cu}$, respectively, an extensive Coulomb excitation program was initiated on $\mathrm{Cu}$ isotopes to investigate the collective and/or single particle features of the low-lying levels in these isotopes. One of the highlights was the first post-accelerated isomeric beam $\left({ }^{68 m} \mathrm{Cu}[80]\right.$ and $\left.{ }^{70 m} \mathrm{Cu}[80,81]\right)$, which illustrated the selectivity of the RILIS. Another highlight was the measured $B(E 2)$ values from the $3 / 2^{-}$ground state to the $5 / 2^{-}$and $1 / 2^{-}$states in ${ }^{67,69,71,73} \mathrm{Cu}$, which indicated a distinct change in the nature of these states beyond $N=40[63]$. The ${ }^{73} \mathrm{Cu}$ beam is a low intensity beam and is heavily contaminated by surface ionized isobaric Ga. The spectra which were shown in fig. 24 illustrate nicely the benefit from laser-on and laser-off measurements, in order to disentangle the contributions from ${ }^{73} \mathrm{Cu}$ and ${ }^{73} \mathrm{Ga}[65]$. The Coulomb excitation spectrum of the $N=50 \mathrm{nu}-$ cleus ${ }^{80} \mathrm{Zn}$ was shown above to highlight the sensitivity of the Miniball setup (see figs. 15 and 29). The $B(E 2$; $2_{1}^{+} \rightarrow 0_{1}^{+}$) transition strength and the energy of the $2_{1}^{+}$ 
Table 3. List of isotopes which have been post-accelerated to Miniball at REX-ISOLDE from 2001 until 2012.

\begin{tabular}{|c|c|c|c|c|c|c|c|c|}
\hline Element & Mass & $T_{1 / 2}$ & Intensity [pps] & Reaction & Sec. Target & Thickness & Target Ion Source & Reference \\
\hline $\mathrm{F}$ & 17 & $64.49(16) \mathrm{s}$ & $1.8 \times 10^{3}$ & $\left(\mathrm{p}, \mathrm{p}^{\prime}\right)$ & $\left(\mathrm{CH}_{2}\right)_{n}$ & $\approx 40 \mu \mathrm{m}$ & $\mathrm{SiC}+\mathrm{MK} 5$ & {$[76]$} \\
\hline $\mathrm{Na}$ & 29 & $44.9(12) \mathrm{ms}$ & & coulex & ${ }^{104} \mathrm{Pd}$ & $4.1 \mathrm{mg} / \mathrm{cm}^{2}$ & $\mathrm{UC}_{x}+$ surface & {$[62]$} \\
\hline $\mathrm{Na}$ & 30 & $48(2) \mathrm{ms}$ & $5 \times 10^{3}$ & coulex & ${ }^{104} \mathrm{Pd}$ & $4.1 \mathrm{mg} / \mathrm{cm}^{2}$ & $\mathrm{UC}_{x}+$ surface & {$[62]$} \\
\hline \multirow{4}{*}{$\mathrm{Mg}$} & \multirow{4}{*}{30} & \multirow{4}{*}{$335(17) \mathrm{ms}$} & & coulex & ${ }^{\text {nat }} \mathrm{Ni}$ & $1.0 \mathrm{mg} / \mathrm{cm}^{2}$ & \multirow{3}{*}{$\mathrm{UC}_{x}+$ RILIS } & {$[77]$} \\
\hline & & & $2 \times 10^{4}$ & $\operatorname{inv}(\mathrm{d}, \mathrm{p})$ & $\mathrm{CD}_{2}$ & $10 \mu \mathrm{m}$ & & {$[78]$} \\
\hline & & & & $\operatorname{inv}(t, p)$ & ${ }^{3} \mathrm{H}$ & $0.04 \mathrm{mg} / \mathrm{cm}^{2}$ & & {$[44]$} \\
\hline & & & & coulex & ${ }^{\text {nat }} \mathrm{Ni}$ & $1 \mathrm{mg} / \mathrm{cm}^{2}$ & $\mathrm{UC}_{x}+$ RILIS & {$[77]$} \\
\hline $\mathrm{Mg}$ & 31 & $232(15) \mathrm{ms}$ & - & coulex & ${ }^{109} \mathrm{Ag}$ & $\begin{array}{l}1.9 \mathrm{mg} / \mathrm{cm}^{2} \\
4.0 \mathrm{mg} / \mathrm{cm}^{2}\end{array}$ & $\mathrm{UC}_{x}+$ RILIS & {$[79]$} \\
\hline $\mathrm{Mg}$ & 32 & $95(16) \mathrm{ms}$ & $1.5 \times 10^{4}$ & coulex & ${ }^{107} \mathrm{Ag}$ & $4.4 \mathrm{mg} / \mathrm{cm}^{2}$ & $\mathrm{UC}_{x}+$ RILIS & {$[27]$} \\
\hline $\mathrm{Ar}$ & 44 & $11.87(5) \mathrm{m}$ & & $\operatorname{inv}(t, p)$ & ${ }^{3} \mathrm{H}$ & & $\mathrm{UC}_{x}+$ VADIS & {$[47]$} \\
\hline Mn & 61 & $0.67(4) \mathrm{s}$ & $1.3 \times 10^{5}$ & coulex & ${ }^{109} \mathrm{Ag}$ & $4.0 \mathrm{mg} / \mathrm{cm}^{2}$ & $\mathrm{UC}_{x}+$ RILIS & {$[22]$} \\
\hline $\mathrm{Mn}$ & 62 & $88(15) \mathrm{ms}$ & $2.3 \times 10^{4}$ & coulex & ${ }^{109} \mathrm{Ag}$ & $4.0 \mathrm{mg} / \mathrm{cm}^{2}$ & $\mathrm{UC}_{x}+$ RILIS & {$[74]$} \\
\hline Mn & 63 & $0.275(4) \mathrm{s}$ & $1.2 \times 10^{4}$ & coulex & ${ }^{109} \mathrm{Ag}$ & $4.0 \mathrm{mg} / \mathrm{cm}^{2}$ & $\mathrm{UC}_{x}+$ RILIS & {$[74]$} \\
\hline $\mathrm{Fe}$ & 61 & $5.98(6) \mathrm{m}$ & $6 \times 10^{3}$ & coulex & ${ }^{109} \mathrm{Ag}$ & $4.0 \mathrm{mg} / \mathrm{cm}^{2}$ & $\mathrm{UC}_{x}+$ RILIS & {$[23]$} \\
\hline $\mathrm{Fe}$ & 62 & $68(2) \mathrm{s}$ & $1.7 \times 10^{4}$ & coulex & ${ }^{109} \mathrm{Ag}$ & $4.0 \mathrm{mg} / \mathrm{cm}^{2}$ & $\mathrm{UC}_{x}+$ RILIS & {$[22]$} \\
\hline $\mathrm{Ni}$ & 66 & $54.6(3) \mathrm{h}$ & $7 \times 10^{6}$ & $\operatorname{inv}(\mathrm{d}, \mathrm{p})$ & $\mathrm{CD}_{2}$ & $\begin{array}{c}100 \mu \mathrm{m} \\
10 \mu \mathrm{m}\end{array}$ & $\mathrm{UC}_{x}+$ RILIS & {$[45]$} \\
\hline $\mathrm{Ni}$ & 66 & $54.6(3) \mathrm{h}$ & & $\operatorname{inv}(\mathrm{d}, \mathrm{p})$ & $\mathrm{CD}_{2}$ & $125 \mu \mathrm{g} / \mathrm{cm}^{2}$ & $\mathrm{UC}_{x}+\mathrm{Ta}+$ RILIS & {$[46]$} \\
\hline $\mathrm{Ni}$ & 66 & $54.6(3) \mathrm{h}$ & & $\operatorname{inv}(t, p)$ & $\mathrm{Ti}+{ }^{3} \mathrm{H}$ & & $\mathrm{UC}_{x}+\mathrm{Ta}+$ RILIS & {$[46]$} \\
\hline $\mathrm{Ni}$ & 68 & $29(2) \mathrm{s}$ & $1 \times 10^{4}$ & coulex & ${ }^{108} \mathrm{Pd}$ & $2.0 \mathrm{mg} / \mathrm{cm}^{2}$ & $\mathrm{UC}_{x}+$ RILIS & {$[69]$} \\
\hline $\mathrm{Cu}$ & 67 & $61.83(12) \mathrm{h}$ & $1.2 \times 10^{5}$ & coulex & $\begin{array}{l}{ }^{120} \mathrm{Sn} \\
{ }^{104} \mathrm{Pd}\end{array}$ & $\begin{array}{l}2.0 \mathrm{mg} / \mathrm{cm}^{2} \\
2.0 \mathrm{mg} / \mathrm{cm}^{2}\end{array}$ & $\mathrm{UC}_{x}+$ RILIS & {$[63]$} \\
\hline $\mathrm{Cu}$ & 68 & $3.75(5) \mathrm{m}$ & $3 \times 10^{5}\left(6^{-}\right)$ & coulex & ${ }^{120} \mathrm{Sn}$ & $2.3 \mathrm{mg} / \mathrm{cm}^{2}$ & $\mathrm{UC}_{x}+$ RILIS & {$[80]$} \\
\hline $\mathrm{Cu}$ & 68 & $31.1(15) \mathrm{s}$ & $-\left(1^{+}\right)$ & coulex & ${ }^{120} \mathrm{Sn}$ & $2.3 \mathrm{mg} / \mathrm{cm}^{2}$ & $\mathrm{UC}_{x}+$ RILIS & {$[80]$} \\
\hline $\mathrm{Cu}$ & 69 & $2.85(15) \mathrm{m}$ & $1.6 \times 10^{5}$ & coulex & $\begin{array}{l}{ }^{120} \mathrm{Sn} \\
{ }^{104} \mathrm{Pd}\end{array}$ & $\begin{array}{l}2.0 \mathrm{mg} / \mathrm{cm}^{2} \\
2.0 \mathrm{mg} / \mathrm{cm}^{2}\end{array}$ & $\mathrm{UC}_{x}+$ RILIS & {$[63]$} \\
\hline $\mathrm{Cu}$ & 70 & $44.5(2) \mathrm{s}$ & $5 \times 10^{4}\left(6^{-}\right)$ & coulex & ${ }^{120} \mathrm{Sn}$ & $2.3 \mathrm{mg} / \mathrm{cm}^{2}$ & $\mathrm{UC}_{x}+$ RILIS & {$[80]$} \\
\hline & & & $9 \times 10^{5}\left(25 \% 3^{-}\right)$ & coulex & ${ }^{120} \mathrm{Sn}$ & $2.3 \mathrm{mg} / \mathrm{cm}^{2}$ & $\mathrm{UC}_{x}+$ RILIS & {$[81]$} \\
\hline $\mathrm{Cu}$ & 71 & $19.5(16) \mathrm{s}$ & $2.3 \times 10^{5}$ & coulex & $\begin{array}{l}{ }^{120} \mathrm{Sn} \\
{ }^{104} \mathrm{Pd}\end{array}$ & $\begin{array}{l}2.0 \mathrm{mg} / \mathrm{cm}^{2} \\
2.0 \mathrm{mg} / \mathrm{cm}^{2}\end{array}$ & $\mathrm{UC}_{x}+$ RILIS & {$[63]$} \\
\hline $\mathrm{Cu}$ & 73 & $4.2(3) \mathrm{s}$ & $8 \times 10^{4}$ & coulex & $\begin{array}{l}{ }^{120} \mathrm{Sn} \\
{ }^{104} \mathrm{Pd}\end{array}$ & $\begin{array}{l}2.0 \mathrm{mg} / \mathrm{cm}^{2} \\
2.0 \mathrm{mg} / \mathrm{cm}^{2}\end{array}$ & $\mathrm{UC}_{x}+$ RILIS & {$[63]$} \\
\hline $\mathrm{Zn}$ & 74 & $95.6(12) \mathrm{s}$ & $3.0 \times 10^{5}$ & coulex & ${ }^{120} \mathrm{Sn}$ & $2.3 \mathrm{mg} / \mathrm{cm}^{2}$ & $\mathrm{UC}_{x}+$ RILIS & {$[75]$} \\
\hline $\mathrm{Zn}$ & 76 & $5.7(3) \mathrm{s}$ & $1.1 \times 10^{5}$ & coulex & ${ }^{120} \mathrm{Sn}$ & $2.3 \mathrm{mg} / \mathrm{cm}^{2}$ & $\mathrm{UC}_{x}+$ RILIS & {$[75]$} \\
\hline $\mathrm{Zn}$ & 78 & $1.47(15) \mathrm{s}$ & $2-4 \times 10^{5}$ & $\operatorname{inv}(\mathrm{d}, \mathrm{p})$ & $\mathrm{CD}_{2}$ & $100 \mu \mathrm{g}$ & $\mathrm{UC}_{x}+\mathrm{Ta}+$ RILIS & \\
\hline $\mathrm{Zn}$ & 78 & $1.47(15) \mathrm{s}$ & $4.3 \times 10^{3}$ & coulex & ${ }^{108} \mathrm{Pd}$ & $2.0 \mathrm{mg} / \mathrm{cm}^{2}$ & $\mathrm{UC}_{x}+$ RILIS & {$[75]$} \\
\hline $\mathrm{Zn}$ & 80 & $0.54(2) \mathrm{s}$ & $3.0 \times 10^{3}$ & coulex & ${ }^{108} \mathrm{Pd}$ & $2.0 \mathrm{mg} / \mathrm{cm}^{2}$ & $\mathrm{UC}_{x}+$ RILIS & {$[75]$} \\
\hline $\mathrm{Ga}$ & 73 & $4.86(3) \mathrm{h} \mathrm{s}$ & $3.8 \times 10^{5}$ & coulex & ${ }^{120} \mathrm{Sn}$ & $1.7 \mathrm{mg} / \mathrm{cm}^{2}$ & $\mathrm{UC}_{x}+$ RILIS & {$[65]$} \\
\hline $\mathrm{Se}$ & 70 & $41.1(3) \mathrm{m}$ & $1 \times 10^{4}$ & coulex & ${ }^{104} \mathrm{Pd}$ & $2.0 \mathrm{mg} / \mathrm{cm}^{2}$ & $\begin{array}{c}\mathrm{ZrO}_{2}+\text { FEBIAD } \\
\text { SeCO molecular beam }\end{array}$ & {$[70]$} \\
\hline $\mathrm{Rb}$ & 93 & $5.84(2) \mathrm{s}$ & $6 \times 10^{6}$ & coulex & $\begin{array}{l}{ }^{60} \mathrm{Ni} \\
{ }^{196} \mathrm{Pt}\end{array}$ & $\begin{array}{l}2.1 \mathrm{mg} / \mathrm{cm}^{2} \\
2.0 \mathrm{mg} / \mathrm{cm}^{2}\end{array}$ & $\begin{array}{l}\mathrm{UC}_{x}+\mathrm{W} \text { surface } \\
\mathrm{UC}_{x}+\mathrm{W} \text { surface }\end{array}$ & $\begin{array}{l}{[82]} \\
{[82]}\end{array}$ \\
\hline
\end{tabular}


Table 3. Continued.

\begin{tabular}{|c|c|c|c|c|c|c|c|c|}
\hline Element & Mass & $T_{1 / 2}$ & Intensity [pps] & Reaction & Sec. Target & Thickness & Target Ion Source & Reference \\
\hline \multirow{2}{*}{$\mathrm{Rb}$} & \multirow{2}{*}{95} & \multirow{2}{*}{$377.7(8) \mathrm{ms}$} & \multirow{2}{*}{$1 \times 10^{6}$} & \multirow{2}{*}{ coulex } & ${ }^{60} \mathrm{Ni}$ & $2.1 \mathrm{mg} / \mathrm{cm}^{2}$ & $\mathrm{UC}_{x}+\mathrm{W}$ surface & {$[82]$} \\
\hline & & & & & ${ }^{196} \mathrm{Pt}$ & $2.0 \mathrm{mg} / \mathrm{cm}^{2}$ & $\mathrm{UC}_{x}+\mathrm{W}$ surface & {$[82]$} \\
\hline \multirow{2}{*}{$\mathrm{Rb}$} & \multirow{2}{*}{97} & \multirow{2}{*}{ 169.1(6) ms } & \multirow{2}{*}{$5 \times 10^{5}$} & \multirow{2}{*}{ coulex } & ${ }^{60} \mathrm{Ni}$ & $2.1 \mathrm{mg} / \mathrm{cm}^{2}$ & $\mathrm{UC}_{x}+\mathrm{W}$ surface & {$[82]$} \\
\hline & & & & & ${ }^{196} \mathrm{Pt}$ & $2.0 \mathrm{mg} / \mathrm{cm}^{2}$ & $\mathrm{UC}_{x}+\mathrm{W}$ surface & {$[82]$} \\
\hline \multirow{2}{*}{$\mathrm{Rb}$} & \multirow{2}{*}{99} & \multirow{2}{*}{$50.3(7) \mathrm{ms}$} & \multirow{2}{*}{$\approx 10^{3}$} & \multirow{2}{*}{ coulex } & ${ }^{60} \mathrm{Ni}$ & $2.1 \mathrm{mg} / \mathrm{cm}^{2}$ & $\mathrm{UC}_{x}+\mathrm{W}$ surface & [82] \\
\hline & & & & & ${ }^{196} \mathrm{Pt}$ & $2.0 \mathrm{mg} / \mathrm{cm}^{2}$ & $\mathrm{UC}_{x}+\mathrm{W}$ surface & {$[82]$} \\
\hline $\mathrm{Kr}$ & 72 & $17.1(2) \mathrm{s}$ & & coulex & ${ }^{104} \mathrm{Pd}$ & $2.0 \mathrm{mg} / \mathrm{cm}^{2}$ & $\mathrm{UC}_{x}+$ VADIS & [83] \\
\hline \multirow{2}{*}{$\mathrm{Kr}$} & \multirow{2}{*}{88} & \multirow{2}{*}{$2.84(3) \mathrm{h}$} & \multirow{2}{*}{$7 \times 10^{6}$} & \multirow{2}{*}{ coulex } & ${ }^{109} \mathrm{Ag}$ & $2.0 \mathrm{mg} / \mathrm{cm}^{2}$ & $\mathrm{UC}_{x}+\mathrm{MK} 7$ & [84] \\
\hline & & & & & ${ }^{12} \mathrm{C}$ & $2.0 \mathrm{mg} / \mathrm{cm}^{2}$ & $\mathrm{UC}_{x}+\mathrm{MK} 7$ & {$[85]$} \\
\hline \multirow{2}{*}{$\mathrm{Kr}$} & 92 & $1840(8) \mathrm{s}$ & $1 \times 10^{6}$ & couley & ${ }^{109} \mathrm{Ag}$ & $2.0 \mathrm{mg} / \mathrm{cm}^{2}$ & $\mathrm{UC}_{x}+\mathrm{MK} 7$ & {$[84,85]$} \\
\hline & 92 & $1.840(8) \mathrm{s}$ & $1 \times 10$ & coulex & ${ }^{196} \mathrm{Pt}$ & $2.0 \mathrm{mg} / \mathrm{cm}^{2}$ & $\mathrm{UC}_{x}+\mathrm{VADIS}$ & {$[86]$} \\
\hline $\mathrm{Kr}$ & 94 & $212(5) \mathrm{ms}$ & $1.7 \times 10^{5}$ & coulex & $\begin{array}{l}{ }^{48} \mathrm{Ti} \\
{ }^{196} \mathrm{Pt}\end{array}$ & $\begin{array}{l}1.4 \mathrm{mg} / \mathrm{cm}^{2} \\
2.0 \mathrm{mg} / \mathrm{cm}^{2}\end{array}$ & $\mathrm{UC}_{x}+\mathrm{VADIS}$ & {$[86]$} \\
\hline $\mathrm{Kr}$ & 96 & $80(6) \mathrm{ms}$ & $2.8 \times 10^{4}$ & coulex & ${ }^{194} \mathrm{Pt}$ & $2.0 \mathrm{mg} / \mathrm{cm}^{2}$ & $\mathrm{UC}_{x}+$ VADIS & {$[25,86]$} \\
\hline $\mathrm{Sr}$ & 96 & $1.07(1) \mathrm{s}$ & $1 \times 10^{4}$ & coulex & $\begin{array}{l}{ }^{109} \mathrm{Ag} \\
{ }^{120} \mathrm{Sn}\end{array}$ & $1.9 \mathrm{mg} / \mathrm{cm}^{2}$ & $\mathrm{UC}_{x}+$ Ta surface & {$[71]$} \\
\hline $\mathrm{Cd}$ & 102 & $5.5(5) \mathrm{m}$ & $5 \times 10^{5}$ & coulex & $\begin{array}{l}{ }^{120} \mathrm{Sn} \\
{ }^{109} \mathrm{Ag} \\
{ }^{64} \mathrm{Zn}\end{array}$ & $\begin{array}{l}2.0 \mathrm{mg} / \mathrm{cm}^{2} \\
1.9 \mathrm{mg} / \mathrm{cm}^{2} \\
1.8 \mathrm{mg} / \mathrm{cm}^{2}\end{array}$ & $\mathrm{LaC}_{x}+$ RILIS & {$[64]$} \\
\hline $\mathrm{Cd}$ & 104 & $57.7(10) \mathrm{m}$ & $1 \times 10^{6}$ & coulex & $\begin{array}{l}{ }^{109} \mathrm{Ag} \\
{ }^{64} \mathrm{Zn}\end{array}$ & $\begin{array}{l}1.9 \mathrm{mg} / \mathrm{cm}^{2} \\
1.8 \mathrm{mg} / \mathrm{cm}^{2}\end{array}$ & $\mathrm{LaC}_{x}+$ RILIS & {$[64]$} \\
\hline $\mathrm{Cd}$ & 100 & $49.1(5) \mathrm{s}$ & $3 \times 10^{3}$ & coulex & ${ }^{109} \mathrm{Ag}$ & $1.9 \mathrm{mg} / \mathrm{cm}^{2}$ & $\operatorname{LaC}_{x}+$ RILIS & {$[64]$} \\
\hline $\mathrm{Cd}$ & 122 & $5.24(3) \mathrm{s}$ & & coulex & ${ }^{104} \mathrm{Pd}$ & $2.0 \mathrm{mg} / \mathrm{cm}^{2}$ & & {$[87]$} \\
\hline $\mathrm{Cd}$ & 124 & $1.25(2) \mathrm{s}$ & $1.0 \times 10^{4}$ & coulex & ${ }^{64} \mathrm{Zn}$ & $1.8 \mathrm{mg} / \mathrm{cm}^{2}$ & $\mathrm{UC}_{x}+$ RILIS & {$[87]$} \\
\hline $\mathrm{Cd}$ & 126 & $0.515(17) \mathrm{s}$ & $1.4 \times 10^{4}$ & coulex & ${ }^{64} \mathrm{Zn}$ & $1.8 \mathrm{mg} / \mathrm{cm}^{2}$ & $\mathrm{UC}_{x}+$ RILIS & {$[87]$} \\
\hline $\mathrm{Cd}$ & 128 & $0.28(4) \mathrm{s}$ & $1.0 \times 10^{4}$ & coulex & ${ }^{64} \mathrm{Zn}$ & $1.8 \mathrm{mg} / \mathrm{cm}^{2}$ & $\mathrm{UC}_{x}+$ Ta surface & {$[88]$} \\
\hline $\mathrm{Sn}$ & 106 & $115(5) \mathrm{s}$ & - & coulex & ${ }^{58} \mathrm{Ni}$ & $2.0 \mathrm{mg} / \mathrm{cm}^{2}$ & LaC $_{x}+$ RILIS & [89] \\
\hline $\mathrm{Sn}$ & 107 & $2.90(5) \mathrm{m}$ & $1.0 \times 10^{5}$ & coulex & ${ }^{58} \mathrm{Ni}$ & $1.95 \mathrm{mg} / \mathrm{cm}^{2}$ & $\mathrm{LaC}_{x}+$ RILIS & {$[90]$} \\
\hline $\mathrm{Sn}$ & 108 & $10.30(8) \mathrm{m}$ & - & coulex & ${ }^{58} \mathrm{Ni}$ & $2.0 \mathrm{mg} / \mathrm{cm}^{2}$ & $\mathrm{LaC}_{x}+$ RILIS & {$[89]$} \\
\hline $\mathrm{Sn}$ & 109 & $18.0(2) \mathrm{m}$ & $1.0 \times 10^{5}$ & coulex & ${ }^{58} \mathrm{Ni}$ & $1.95 \mathrm{mg} / \mathrm{cm}^{2}$ & $\operatorname{LaC}_{x}+$ RILIS & {$[91]$} \\
\hline $\mathrm{Sn}$ & 110 & $4.11(10) \mathrm{h}$ & $1 \times 10^{6}$ & coulex & ${ }^{58} \mathrm{Ni}$ & $2.0 \mathrm{mg} / \mathrm{cm}^{2}$ & $\operatorname{LaC}_{x}+$ RILIS & {$[92]$} \\
\hline $\mathrm{Xe}$ & 138 & $14.08(8) \mathrm{m}$ & $5 \times 10^{6}$ & coulex & ${ }^{96} \mathrm{Mo}$ & $1.7 \mathrm{mg} / \mathrm{cm}^{2}$ & $\mathrm{UC}_{x}+\mathrm{MK} 7$ & {$[87]$} \\
\hline $\mathrm{Xe}$ & 140 & $13.60(10) \mathrm{s}$ & $3 \times 10^{6}$ & coulex & ${ }^{96} \mathrm{Mo}$ & $1.7 \mathrm{mg} / \mathrm{cm}^{2}$ & $\mathrm{UC}_{x}+\mathrm{MK} 7$ & {$[87]$} \\
\hline $\mathrm{Xe}$ & 142 & $1.22(2) \mathrm{s}$ & $3 \times 10^{6}$ & coulex & ${ }^{96} \mathrm{Mo}$ & $1.7 \mathrm{mg} / \mathrm{cm}^{2}$ & $\mathrm{UC}_{x}+\mathrm{MK} 7$ & {$[87]$} \\
\hline $\mathrm{Xe}$ & 144 & $1.15(20) \mathrm{s}$ & $5 \times 10^{4}$ & coulex & ${ }^{96} \mathrm{Mo}$ & $1.7 \mathrm{mg} / \mathrm{cm}^{2}$ & $\mathrm{UC}_{x}+\mathrm{MK} 7$ & {$[87]$} \\
\hline $\mathrm{Ba}$ & 140 & $12.7527(23) \mathrm{d}$ & & coulex & ${ }^{96} \mathrm{Mo}$ & $0.9 \mathrm{mg} / \mathrm{cm}^{2}$ & $\mathrm{UC}_{x}$ & {$[73]$} \\
\hline $\mathrm{Ba}$ & 142 & $10.6(2) \mathrm{m}$ & & coulex & ${ }^{96} \mathrm{Mo}$ & $0.9 \mathrm{mg} / \mathrm{cm}^{2}$ & $\mathrm{UC}_{x}$ & {$[73]$} \\
\hline $\mathrm{Hg}$ & 182 & $10.83(6) \mathrm{s}$ & $4.9 \times 10^{3}$ & coulex & ${ }^{112} \mathrm{Cd}$ & $2.0 \mathrm{mg} / \mathrm{cm}^{2}$ & molten $\mathrm{Pb}+\mathrm{MK} 3$ & [93] \\
\hline $\mathrm{Hg}$ & 184 & $30.87(26) \mathrm{s}$ & $1.0 \times 10^{5}$ & coulex & ${ }^{112} \mathrm{Cd}$ & $2.0 \mathrm{mg} / \mathrm{cm}^{2}$ & molten $\mathrm{Pb}+\mathrm{MK} 3$ & [93] \\
\hline $\mathrm{Hg}$ & 186 & $1.38(6) \mathrm{m}$ & $2.5 \times 10^{5}$ & coulex & ${ }^{114} \mathrm{Cd}$ & $2.0 \mathrm{mg} / \mathrm{cm}^{2}$ & molten $\mathrm{Pb}+\mathrm{MK} 3$ & {$[93]$} \\
\hline $\mathrm{Hg}$ & 188 & $3.25(15) \mathrm{m}$ & $3.1 \times 10^{5}$ & coulex & ${ }^{114} \mathrm{Cd}$ & $2.0 \mathrm{mg} / \mathrm{cm}^{2}$ & molten $\mathrm{Pb}+\mathrm{MK} 3$ & [93] \\
\hline $\mathrm{Pb}$ & 188 & $24(2) \mathrm{s}$ & $2.8 \times 10^{5}$ & coulex & ${ }^{112} \mathrm{Cd}$ & $2.0 \mathrm{mg} / \mathrm{cm}^{2}$ & $\mathrm{UC}_{x}+$ RILIS & {$[94]$} \\
\hline $\mathrm{Pb}$ & 190 & $1.2(1) \mathrm{m}$ & $1.4 \times 10^{6}$ & coulex & ${ }^{112} \mathrm{Cd}$ & $2.0 \mathrm{mg} / \mathrm{cm}^{2}$ & $\mathrm{UC}_{x}+$ RILIS & {$[94]$} \\
\hline $\mathrm{Pb}$ & 192 & $3.5(1) \mathrm{m}$ & $5.0 \times 10^{5}$ & coulex & ${ }^{112} \mathrm{Cd}$ & $2.0 \mathrm{mg} / \mathrm{cm}^{2}$ & $\mathrm{UC}_{x}+$ RILIS & {$[94]$} \\
\hline $\mathrm{Pb}$ & 194 & $12.0(5) \mathrm{m}$ & $1.3 \times 10^{6}$ & coulex & ${ }^{112} \mathrm{Cd}$ & $2.0 \mathrm{mg} / \mathrm{cm}^{2}$ & $\mathrm{UC}_{x}+$ RILIS & {$[94]$} \\
\hline $\mathrm{Pb}$ & 196 & $37(3) \mathrm{m}$ & $5.4 \times 10^{5}$ & coulex & ${ }^{112} \mathrm{Cd}$ & $2.0 \mathrm{mg} / \mathrm{cm}^{2}$ & $\mathrm{UC}_{x}+$ RILIS & {$[94]$} \\
\hline $\mathrm{Pb}$ & 198 & $2.40(10) \mathrm{h}$ & $1-5 \times 10^{5}$ & coulex & ${ }^{112} \mathrm{Cd}$ & $2.0 \mathrm{mg} / \mathrm{cm}^{2}$ & $\mathrm{UC}_{x}+$ RILIS & {$[94]$} \\
\hline $\mathrm{Po}$ & 200 & $11.51(8) \mathrm{m}$ & $2.0 \times 10^{6}$ & coulex & ${ }^{104} \mathrm{Pd}$ & $2.0 \mathrm{mg} / \mathrm{cm}^{2}$ & $\mathrm{UC}_{x}+$ RILIS & {$[26,95]$} \\
\hline $\mathrm{Rn}$ & 202 & $9.7(1) \mathrm{s}$ & $3 \times 10^{4}$ & coulex & ${ }^{109} \mathrm{Ag}$ & $4.0 \mathrm{mg} / \mathrm{cm}^{2}$ & $\mathrm{UC}_{x}+\mathrm{MK} 7$ & \\
\hline $\mathrm{Rn}$ & 204 & $74.5(14) \mathrm{s}$ & $2 \times 10^{5}$ & coulex & $\begin{array}{l}{ }^{109} \mathrm{Ag} \\
{ }^{120} \mathrm{Sn}\end{array}$ & $\begin{array}{l}1.9 \mathrm{mg} / \mathrm{cm}^{2} \\
2.0 \mathrm{mg} / \mathrm{cm}^{2}\end{array}$ & $\mathrm{UC}_{x}+\mathrm{MK} 7$ & {$[96]$} \\
\hline $\mathrm{Rn}$ & 208 & $23.35(14) \mathrm{m}$ & $5.6 \times 10^{5}$ & coulex & ${ }^{112} \mathrm{Cd}$ & $2.0 \mathrm{mg} / \mathrm{cm}^{2}$ & $\mathrm{UC}_{x}+$ VADIS & \\
\hline $\mathrm{Ra}$ & 224 & $3.66(4) \mathrm{d}$ & $4.2 \times 10^{5}$ & coulex & $\begin{array}{l}{ }^{112} \mathrm{Cd} \\
{ }^{120} \mathrm{Sn}\end{array}$ & $\begin{array}{l}2.0 \mathrm{mg} / \mathrm{cm}^{2} \\
2.0 \mathrm{mg} / \mathrm{cm}^{2}\end{array}$ & $\mathrm{UC}_{x}$ & {$[24]$} \\
\hline
\end{tabular}


state in this nucleus provide crucial information to understand the development of collectivity around ${ }^{78} \mathrm{Ni}$. This experimental result was used to fix the $Z=28$ shell gap in a recently developed new residual interaction in this mass region [102], that predicts the appearance of a new "island of inversion" south of ${ }^{68} \mathrm{Ni}$, namely in the neutron-rich Cr isotopes. Recently, d $\left({ }^{66} \mathrm{Ni},{ }^{67} \mathrm{Ni}\right) \mathrm{p}, \mathrm{t}\left({ }^{66} \mathrm{Ni},{ }^{68} \mathrm{Ni}\right) \mathrm{p}$ and $\mathrm{d}\left({ }^{78} \mathrm{Zn},{ }^{79} \mathrm{Zn}\right) \mathrm{p}$ transfer experiments were performed with the T-REX setup to probe the single-particle properties of ${ }^{67,68} \mathrm{Ni}$ and ${ }^{79} \mathrm{Zn}$, respectively. The data are currently under analysis.

\subsubsection{The region around ${ }^{100} \mathrm{Sn}$}

The isotopes between ${ }^{100} \mathrm{Sn}$ and ${ }^{132} \mathrm{Sn}$ provide a uniquely long chain of semi-magic isotopes that can be used to test nucleon-nucleon interactions based on first principles. Historically, the even-even isotopes of this chain have been a textbook example for the seniority scheme with almost constant $2_{1}^{+}$energies. A recent review on this region can be found in [98]. Large-scale seniority truncated shell-model calculations have also well reproduced the trend of experimental $B\left(E 2 ; 0^{+} \rightarrow 2^{+}\right)$values for the even isotopes from mid-shell to the heavier masses. Although the structure is more complex, the observed trend and the theoretical predictions have largely resembled what could be expected from the simple seniority model based on a single j-shell. However, the lifetimes of the lowest-lying states in the neutron-deficient even-even isotopes have not been accessible experimentally due to the isomeric nature of the $6^{+}$states in these isotopes. As expected, the structure of the $6_{1}^{+}$state is predicted to be dominated by recoupling of two neutrons occupying the two lowest-lying orbitals above the $N=50$ shell-gap; i.e. the $\mathrm{d}_{5 / 2}$ and $\mathrm{g}_{7 / 2}$ orbitals, to a maximum spin. Here one can note that the ordering of the $d_{5 / 2}$ and $g_{7 / 2}$ orbitals is still a subject of debate and that the location of the other single-particle orbitals in ${ }^{101} \mathrm{Sn}$ is not known. The $6^{+}$states have been used in earlier work to deduce the extent of core excitation via effective charge determinations. In a series of experiments using Miniball at REX-ISOLDE, the lifetimes of the first $2^{+}$states in ${ }^{110,108,106} \mathrm{Sn}$ have now been measured using Coulomb excitation [89,92] in order to circumvent the isomerism of the $6^{+}$states. A clear deviation from the large-scale shell-model calculations mentioned above has been observed. The results have also been confirmed by high-energy Coulomb excitation measurements at GSI and NSCL and have also inspired new measurements in the mid-shell region. A new more complex picture of the excitation probabilities, sensitive to the location of the single-particle orbitals as well as to core-excitation effects, has started to emerge. Just as in the ${ }^{78} \mathrm{Ni}$ region, the experiments in this region have relied on the selectivity of the ISOLDE RILIS and on the development of new primary targets. In complementary experiments on ${ }^{104,102,100} \mathrm{Cd}$, quadrupole deformations and transition matrix elements have also been measured [64].

\subsection{Shape co-existence}

Because of the sensitivity of the differential Coulomb excitation cross section to the sign and magnitude of the quadrupole moment of the excited states, multistep Coulomb excitation can be used to probe shape coexistence. At REX-ISOLDE, the cross sections for multistep Coulomb excitation are low given the beam energy of only $3 \mathrm{MeV} / A$. Nevertheless, information on the sign of the quadrupole moment of excited states can still be inferred, when the lifetime of the state is known (for a recent review, see [103]).

\subsubsection{The region around $Z \approx 40$}

Shape co-existence in Se and $\mathrm{Sr}$ isotopes has been probed by Coulomb excitation of ${ }^{70} \mathrm{Se}$ and ${ }^{96} \mathrm{Sr}$. In both cases, a pure beam of ${ }^{70} \mathrm{Se}$ and ${ }^{96} \mathrm{Sr}$ was obtained by using the molecular extraction technique, described in sect. 6.5. The low statistics in both experiments did not enable the extraction of the quadrupole moment of the first excited $2^{+}$ state based on a differential cross section measurement. Both experiments utilized the available lifetime of the $2_{1}^{+}$ state to gain information on the quadrupole moment. The need for accurate lifetime measurements is clear from two publications related to the deformation in ${ }^{70} \mathrm{Se}[70,104]$.

In the same mass region, Miniball experiments have determined the $B\left(E 2 ; 0_{1}^{+} \rightarrow 2_{1}^{+}\right)$values for ${ }^{88,92,94,96} \mathrm{Kr}[25$, $84,86]$ and in ${ }^{96} \mathrm{Kr}$ the discrepancy between the previously published $2^{+}$energy [105] and mass measurements [106], which provided contradictory information about the deformation of this nucleus, could be resolved.

In order to explore the borders of the island of sudden onset of deformation around $N=60$ a Coulomb excitation study of the odd-mass ${ }^{93,95,97,99} \mathrm{Rb}$ isotopes was performed [82]. It has demonstrated a clear distinction between the spherical shapes below $N=60\left({ }^{93} \mathrm{Rb}\right.$ and $\left.{ }^{95} \mathrm{Rb}\right)$ and well deformed structures right above it $\left({ }^{97} \mathrm{Rb}\right.$ and $\left.{ }^{99} \mathrm{Rb}\right)$. The results are presently in preparation [107].

\subsubsection{The region around $Z \approx 82$}

The interplay between single-particle motion, collectivity, and pairing in the vicinity of light $\mathrm{Pb}$ nuclei is manifested as a rich tapestry of coexisting nuclear shapes and exotic excitations $[108,109]$. One of the goals of modern nuclear physics research is to understand the origin of these structures and their relation to the fundamental interactions between the nuclear constituents. These subjects can be investigated particularly well in the very neutron-deficient $Z \approx 82$ isotopes, where a relatively small proton shell gap, together with a large valence neutron space, provides fertile ground for studies of shape transitions within a small energy range. Over decades, a considerable body of both theoretical and experimental evidence has been gathered and a whole arsenal of spectroscopic techniques has been developed to investigate 
this phenomenon. Very recently, heavy isotopes in this region (i.e. ${ }_{80} \mathrm{Hg},{ }_{82} \mathrm{~Pb},{ }_{84} \mathrm{Po}, \ldots$ ) have become available as radioactive ion beams. When post-accelerated up to $\approx 3 \mathrm{MeV} / A$, multi-step Coulomb excitation can be performed. Combining with additional information on lifetimes in these isotopes, a complete setup of matrix elements can be extracted from these experiments, providing insight into the deformation and mixing of the low-lying excited states.

An intense research program has been initiated at REX-ISOLDE, where intense beams of $\mathrm{Hg}, \mathrm{Pb}, \mathrm{Po}$ and $\mathrm{Rn}$ are available. Except for Rn, these beams use the RILIS ion source, which is essential for the beam purity. The $\mathrm{Hg}$ beams are uniquely produced with a molten $\mathrm{Pb}$ target, a concept that is described in [110]. These unique lowenergy beams at REX-ISOLDE have so far yielded results for Coulomb-excitation experiments on ${ }^{182,184,186,188} \mathrm{Hg}$, ${ }^{188,190,192,194,196,198} \mathrm{~Pb},{ }^{200} \mathrm{Po}$ and ${ }^{202,204} \mathrm{Rn}$, allowing for systematic studies of the competing structures in this region. In most of these cases, the differential cross section could be measured thanks to the selective kinematics, where both the target-like ejectile as the beam-like projectile are simultaneously detected in the DSSSD. The inverse kinematics enables the selection of regions in the center of mass frame, where the sensitivity to the quadrupole moment is different.

\section{Selected results obtained with Miniball at other facilities}

In this section, the use of the Miniball detectors for inflight experiments at GSI (Gesellschaft für Schwerionenforschung in Darmstadt, Germany) and at the ISOL facility LISOL (Leuven Isotopes Separator On-Line at the Centre de Recherche Cyclotron of Louvain-la-Neuve, Belgium) is described.

\section{$9.1 \mathrm{GSI}$}

The Miniball detectors have been used at two different positions at the Fragment Separator (FRS) at GSI: as part of a larger array (the "RISING" array [111]) in the final focal plane (S4) and in the intermediate focal plane (S2). The configuration and performance of the detectors in the two positions will be described below.

\subsubsection{Miniball at the RISING fast-beam setup}

The RISING fast-beam campaign at GSI focused on inbeam $\gamma$-ray spectroscopy experiments with unstable secondary ion beams, produced by high-energy fragmentation or fission of an intense stable primary beam. The availability of secondary beams of exotic nuclei with energies up to $1 \mathrm{GeV} / A$ and with atomic numbers up to $Z=92$ for relativistic Coulomb excitation and two-step fragmentation are unprecedented for in-beam $\gamma$-ray spectroscopy.
High-resolution $\gamma$-ray spectroscopy at relativistic beam energies is challenging and limitations imposed by large Doppler effects and background caused by atomic processes and unwanted nuclear interactions have to be considered. For this purpose the incoming and outgoing particles of the secondary target have to be identified in mass and charge on an event-by-event basis. Also the kinetic energy, the velocity and the trajectory have to be determined. The design of the germanium detector array was optimised to achieve the highest possible efficiency and energy resolution. A set of heavy-ion detectors are located in front and behind the secondary target in order to provide best spatial position resolution, timing properties and energy resolution for the individual heavy ions. Especially the trajectory is crucial for scattering angle determination. This is most relevant for the Doppler correction. Moreover, the proper range of impact parameter is selected for Coulomb excitation at relativistic energies.

A dedicated array of $\gamma$-ray detectors (the RISING array [111]) was built at the final focal plane of the FRS at GSI around the secondary target. The RISING Fast Beam array was designed to measure photons in flight at beam velocities $\beta \geq 0.4$. The setup consisted of three different $\gamma$-ray detector arrays: fifteen former EUROBALL Ge cluster detectors [10], the eight Miniball Ge cluster detectors and eight $\mathrm{BaF}_{2}$ detectors (the HECTOR array) [112]. Each EUROBALL cluster detector is composed of seven closely packed tapered hexagonal Ge crystal. The geometry of the EUROBALL crystals is identical to the Miniball crystals, but without the six-fold segmentation of the Miniball crystals (see sect. 2). An add-back routine, as described in sect. 2.7, was performed on both the Miniball and EUROBALL cluster detectors. Due to the high beam velocity, the observed $\gamma$-ray energies are strongly Doppler shifted and must be corrected according to eq. (4). As can be seen from fig. 27 the $\gamma$-ray angular distribution is strongly peaked at forward angles at these velocities. The large effects of the Lorentz transformation on the efficiency and energy resolution of the RISING detectors were simulated in detail. These simulations were used to optimize the arrangement of the different detector arrays. The EUROBALL cluster detectors were mounted in three rings around the beam pipe at angles of $16^{\circ}, 33^{\circ}$, and $36^{\circ}$ with variable distances of 700 to $1400 \mathrm{~mm}$. For this geometry, an energy resolution of $1.6 \%$ and a full-energy photopeak detection efficiency of $\epsilon_{\text {peak }}=2.8 \%$ for a $1.3 \mathrm{MeV} \gamma$-ray emitted from a moving nucleus at $100 \mathrm{MeV} / A(\beta=0.43)$ were calculated [111]. All eight Miniball detectors were mounted in the RISING setup in two rings at central angles of $51^{\circ}$ and $86^{\circ}$. The distance to the target could be varied individually between 200 and $400 \mathrm{~mm}$. To shield the Miniball detectors from atomic background produced in the slowing down process of heavy ions inside the target, the exterior of the beam pipe was covered with $1 \mathrm{~mm}$ lead and $2 \mathrm{~mm}$ tin. All signals from the RISING array were fed into XIA DGF-4C modules [12], which provide individual time stamping of all events.

The full-energy photopeak efficiency $\epsilon_{\text {peak }}$ at a beam energy of $100 \mathrm{MeV} / A$ and varying $\gamma$-ray energies between 250 and $3000 \mathrm{keV}$ is shown in fig. 30 in steps of $250 \mathrm{keV}$. 


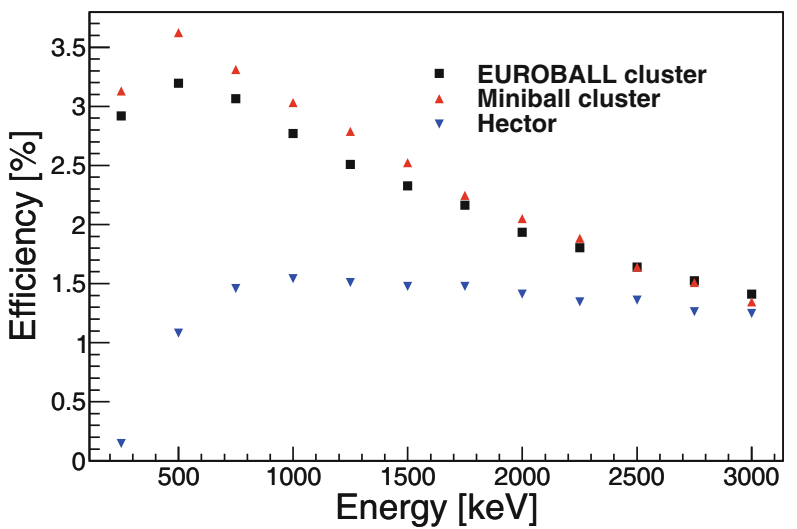

Fig. 30. (Color online) Simulated $\gamma$-ray efficiency $\epsilon_{\text {peak }}$ at a beam energy of $100 \mathrm{MeV} / A$ for the EUROBALL, Miniball and Hector arrays in the RISING fast beam setup [113].

For lower energies the $\gamma$-ray efficiency diminished due to the atomic background absorbers put in front of the detectors and the beam pipe. The efficiency of the 24 Miniball capsules was slightly better than that of the more distant 105 EUROBALL capsules. The high segmentation of the Miniball detectors yields a better resolution compared to the EUROBALL detectors, though the EUROBALL detectors are positioned in forward direction and have to deal with larger Doppler shifts. For the Doppler correction, the angular coordinates of the particles were determined from Si detectors placed after the secondary targets in coincidence with the CATE detector [114]. The angular coordinates of the $\gamma$-rays could be accurately deduced from the segmented germanium detectors. However, the final resolution of the Doppler-corrected transitions is limited by the finite opening angle of the individual germanium crystals.

The changes in energy resolution and in photo peak efficiency were studied for the Miniball under the condition of single hit and add-back events, respectively. In the spectrum of a segment multiplicity equal to one, scattered $\gamma$-rays that do not release their full energy show a Compton edge of the same height as the photo peak. By selecting only events of a segment multiplicity equal to two or higher a considerable part of Compton scattered, singleand double-escape events are discriminated. For a $\gamma$-ray energy of $3 \mathrm{MeV}$ from ${ }^{36} \mathrm{Ca}$ at beam energy of $196 \mathrm{MeV} / A$, the quality of the spectrum, expressed by the $P / T$ ratio improves. The $P / T$ ratio increases from 0.1 for single hit events to 0.2 for the add-back case. About half of the peak integral is lost if the add-back case is separated from single hit events.

In order to highlight the performance of the RISING array, an example of the $2_{1}^{+} \rightarrow 0_{\text {g.s. }}^{+}$decay in ${ }^{36} \mathrm{Ca}$ is shown in fig. 31 [115]. The $2_{1}^{+} \rightarrow 0_{\text {g.s. }}^{+}$transition was observed at an energy of $3014(17) \mathrm{keV}$ with a resolution of $4.3(6) \%$ in the EUROBALL cluster detectors and at $3017(18) \mathrm{keV}$ with a resolution of $4.9(10) \%$ in the Miniball detectors. These resolutions were close to the simulated values of $3.8 \%$ and $4.5 \%$. For the combined Ge detectors, the statistical error was determined to be $15 \mathrm{keV}$, which
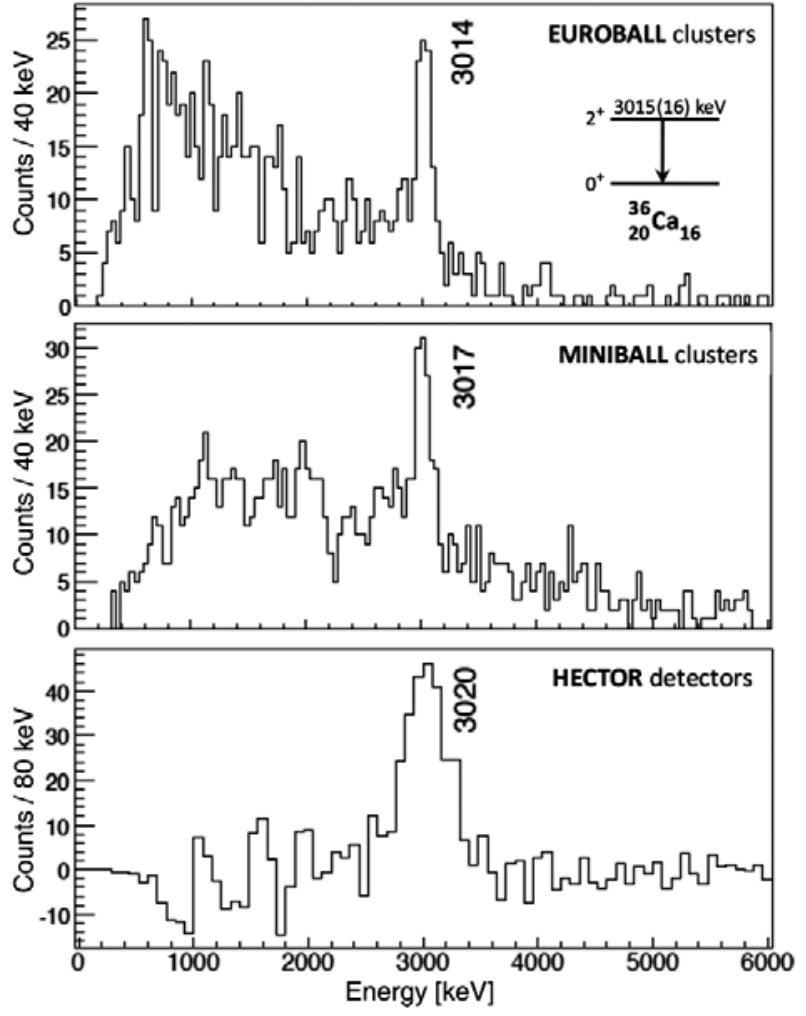

Fig. 31. Doppler corrected ${ }^{36}$ Ca gated $\gamma$-ray spectra measured with the EUROBALL (top), Miniball (middle) and HECTOR (bottom) detectors. For (bottom) the background was subtracted [115].

yielded, including an error for observed known high energy transitions of $5 \mathrm{keV}$, an assignment of the $2_{1}^{+} \rightarrow 0_{\text {g.s. }}^{+}$. transition in ${ }^{36} \mathrm{Ca}$ to $3015(16) \mathrm{keV}$. The peak observed at $3020(20) \mathrm{keV}$ for the HECTOR array was in good agreement with this value.

\subsubsection{Miniball at the FRS intermediate focus (S2)}

In two experiments at the FRS, the Miniball spectrometer was positioned around the intermediate focus point of the FRS (S2). The aim of these experiments was to use the FRS as a two-stage spectrometer. The fragments of interest resulting from the fragmentation of the primary beam on the first production target were selected and identified in the first part of the FRS. At the intermediate focus (S2) of the FRS, they impinged on a secondary reaction target $\left(1720 \mathrm{mg} / \mathrm{cm}^{2}{ }^{9} \mathrm{Be}\right)$, where prompt $\gamma$-rays emitted by the reaction products were detected by the Miniball cluster detectors. Subsequently, the secondary reaction products were transported to the final focus (S4) and, on an eventby-event basis, identified. The FRS was operated in energy loss mode, where the dispersion of the first spectrometer stage is matched by the dispersion of the second one. Therefore an energy (momentum) change in the knockout target could be measured with the second stage, independently of the energy (momentum) spread of the incoming beam. This enabled a high-resolution measurement of the parallel and longitudinal momentum distributions of the 
secondary reaction products following the knock-out reaction in S2. The Miniball detectors provided the possibility to tag the individual ions in $\mathrm{S} 4$ with prompt $\gamma$-rays. The Miniball detectors were positioned forward of the secondary reaction target at $\mathrm{S} 2$ in a ring with an average distance of $26.4 \mathrm{~cm}$ between the front face of the detector and the center of the target and at an average angle of $40^{\circ}$ with respect to the beam axis. The absolute full-energy photopeak efficiency in the laboratory frame was determined to be $3.1 \%$ at $344.3 \mathrm{keV}$ and $1.5 \%$ at $1332 \mathrm{keV}$. Using the $6-$ fold segmentation of the Miniball HPGe crystals for the Doppler correction of the $\gamma$-rays emitted by the reaction products at relativistic velocities with $\beta \approx 0.7$, a resolution of $\approx 40 \mathrm{keV}(\mathrm{FWHM})$ at a laboratory $\gamma$-ray energy of $\approx 580 \mathrm{keV}$ was achieved.

The first experiment aimed at the investigation of shell structure near the predicted new doubly magic shell closure in ${ }^{54} \mathrm{Ca}$. One neutron-knockout reactions from ${ }^{56} \mathrm{Ti}$, ${ }^{50} \mathrm{Ca}$ and ${ }^{52,53,54} \mathrm{Sc}$ to ${ }^{55} \mathrm{Ti},{ }^{49} \mathrm{Ca}$ and ${ }^{51,52,53} \mathrm{Sc}$, respectively, were studied. The primary fragments were produced by the fragmentation of a $500 \mathrm{MeV} / A{ }^{86} \mathrm{Kr}$ primary beam with an intensity of up to $10^{9}$ particles per second in a $1625 \mathrm{mg} / \mathrm{cm}^{2}{ }^{9}$ Be production target at the entrance of the FRS. Some of the physics highlights include the evidence of the $7 / 2^{-} \nu f_{7 / 2}$-hole state at $3.35(15) \mathrm{MeV}$ in ${ }^{49} \mathrm{Ca}$ and the determination of the ground state of ${ }^{55} \mathrm{Ti}$ to be a $1 / 2^{-}$state, in agreement with shell-model predictions taking into account effects of the tensor interaction [116].

In the second experiment, the evolution of structure close to the neutron drip line in the region between $\mathrm{C}$ and $\mathrm{Al}$ was investigated by one-neutron knock-out reactions. In this case a $700 \mathrm{MeV} / A{ }^{40} \mathrm{Ar}$ primary beam with an intensity of about $10^{10}$ ions/spill was used to produce neutron-rich fragments. Momentum distributions and cross-sections of the knockout reactions of a number of neutron-rich nuclei were measured. For some nuclei the Miniball $\gamma$-ray data have revealed new excited states. As an example, the experiment provided indications that ${ }^{22} \mathrm{~N}$ may indeed be a halo nucleus [117].

\subsection{LISOL}

At the Leuven Isotope Separator On Line facility (Belgium), beams of short-lived radioactive isotopes are produced using resonant laser ionization in a buffer gas cell [118]. This technique allows for the production of short-lived purified radioactive beams from elements that are, due to their physico-chemical properties, not available from thick target ion source systems like ISOLDE. The drawback is that thin production targets (typically only a few $\mathrm{mg} / \mathrm{cm}^{2}$ ) have to be used as the reaction products have to recoil out of the target in order to be thermalized in the buffer gas and subsequently resonantly laser ionized. Using this production method, beams of neutron-rich iron $(Z=26)$, cobalt $(Z=27)$ and nickel $(Z=28)$ around $N=40$ were produced in proton induced fission of natural uranium. After laser ionization and mass separation, the low-energy radioactive ion beam was implanted into an aluminized Mylar ${ }^{\circledR}$ tape in the middle of a new $\beta-\gamma$ detection system, which is extensively described in [119]. In
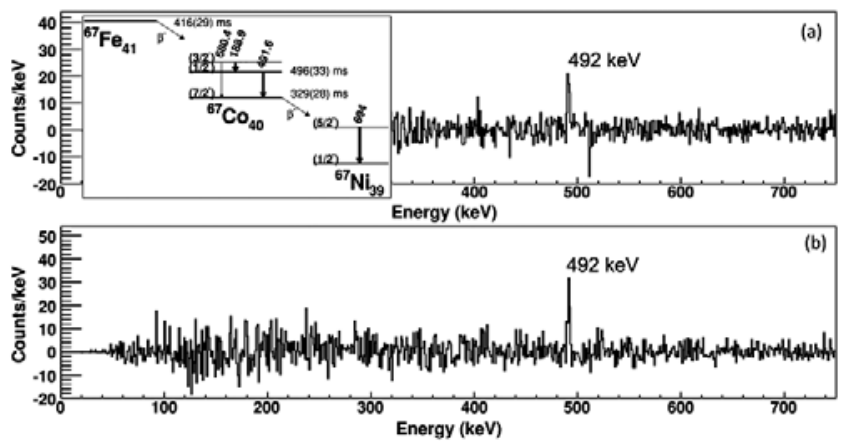

Fig. 32. Random-substracted single $\gamma$-ray events detected after $\beta$-gated $189 \mathrm{keV}$ events in a $1 \mu \mathrm{s}$ to $200 \mathrm{~ms}$ time window (a) and random-subtracted single $\gamma$-ray events detected before $\beta$-gated $694 \mathrm{keV}$ events in a similar time window (b). The presence of the $492 \mathrm{keV}$ line is clearly established. The inset shows the relevant decay schemes.

this setup, two Miniball clusters are placed opposite each other, facing the implantation point (see fig. 1 in [119]) resulting in a solid-angle coverage for $\gamma$ detection of about $54 \%$ of $4 \pi$. Thanks to the segmentation of the Miniball crystals, a net gain in photo-peak efficiency was established due to a strong reduction of true coincidence summing. The probability of two coincident $\gamma$-rays entering the same crystal was $0.8 \%$ for the Miniball setup, while it was $9 \%$ for an older setup, used at the same facility, using two coaxial Germanium detectors [120]. With this Miniball setup a full-energy photopeak efficiency of $5.8 \%$ for a multiplicity $1 \gamma$-ray of $1332 \mathrm{keV}$ was reached. This new setup was used to study the $\beta$ decay of ${ }^{65,66,67} \mathrm{Fe}$, revealing the structure of their daughter nuclei ${ }^{65,66,67} \mathrm{Co} .{ }^{66,67} \mathrm{Co}$ are interesting nuclei, as they are the one-neutron hole one-proton hole $\left(\nu^{-1} \pi^{-1}\right)$ and one-proton hole $\left(\pi^{-1}\right)$ partners of ${ }^{68} \mathrm{Ni}(Z=28, N=40)$, respectively [121]. These nuclei provide important information on the strength of the $Z=28$ and $N=40$ (sub-) shell closures. In the case of the decay of the mass 67 chain, the use of Miniball detectors combined with weak but pure sources allowed a new slow correlation technique to be used, whereby within an extended time window (up to one second), correlations were identified between single $\beta$ and $\gamma$ events and $\beta$-coincident $\gamma$ events. This method evidenced the existence of a long-lived isomer in ${ }^{67} \mathrm{Co}$ at an excitation energy of $492 \mathrm{keV}$. Characteristic prompt $\beta-\gamma(189 \mathrm{keV})$ coincidence events from the $\beta$ decay of ${ }^{67} \mathrm{Fe}\left(T_{1 / 2}=416 \mathrm{~ms}\right)$ were followed by $492 \mathrm{keV}$ transitions observed in the singles $\gamma$-ray spectrum. Figure 32(a) shows the correlated singles $\gamma$-ray spectrum. Furthermore, the $492 \mathrm{keV}$ transition was followed by characteristic prompt $\beta-\gamma(694 \mathrm{keV})$ events from the $\beta$ decay of ${ }^{67} \mathrm{Co}\left(T_{1 / 2}=329 \mathrm{~ms}\right.$ ) (see fig. 32(b)). This firmly established the existence of a longlived isomer in ${ }^{67} \mathrm{Co}$. From the intensity of the $492 \mathrm{keV}$ line as a function of the correlation time window, a halflife value of $496(33) \mathrm{ms}$ was determined and a detailed analysis suggested a $\left(1 / 2^{-}\right)$spin and parity for this isomer. This state was interpreted as a $\pi(1 \mathrm{p}-2 \mathrm{~h})$ excitation across the $Z=28$ shell gap [122] and has been confirmed 
by mass measurements [123]. The new detection setup including two Miniball clusters has been used for further studies at the low-energy branch of the ISOLDE facility at CERN to study the $\beta$ decay of neutron-rich manganese isotopes including ${ }^{68} \mathrm{Mn}[124]$.

\section{Conclusion}

In the past decade, the combination of the low-multiplicity germanium array Miniball and the post-accelerator REXISOLDE has proved to be a unique combination, which has provided a rich and varied physics outcome. A summary was provided of all the post-accelerated beams, from $Z=9$ up to $Z=88$, up to $3 \mathrm{MeV} / A$, which have been used in scattering experiments (Coulomb excitation and/or transfer reactions). The beams range from ${ }^{17} \mathrm{~F}$ to ${ }^{224} \mathrm{Ra}$. The characteristics and performance of the Miniball setup have been described in detail and illustrated by online results. It was shown that experiments have been performed at the limit of feasibility at the current facility: experiments with extremely weak and heavily contaminated radioactive ion beams (e.g., ${ }^{80} \mathrm{Zn}$ Coulomb excitation) and/or small cross sections, which require the highest level of sensitivity and selectivity (e.g., t $\left.\left({ }^{30} \mathrm{Mg},{ }^{32} \mathrm{Mg}\right) \mathrm{p}\right)$.

In the coming years, the current REX-ISOLDE facility will be upgraded to provide higher-intensity and higherenergy post-accelerated beams. In a first stage energies up to $5 \mathrm{MeV} / A$ will be available and in a later stage up to $10 \mathrm{MeV} / A$. This HIE-ISOLDE (High Intensity and Energy ISOLDE) project is foreseen to be completed by 2016. With the availability of higher-energy RIBs, multistep Coulomb-excitation experiments and few-nucleon transfer reactions studies of heavier (i.e. higher $Z$ ) isotopes will become possible. In order to study fusionevaporation reactions, it is intended to equip Miniball with Compton-suppression shielding. Additional experimental equipment, such as a recoil separator, to be combined with the Miniball germanium array and to enhance the sensitivity and selectivity are currently under discussion.

The authors would like to acknowledge financial support from the following agencies: the German BMBF under contracts 06-K167，06-KY205I，06-OK668，06-OK862I，06-OK958，06KY9136I, 06-BA115, 06-MT238, 06-MT9156, 06-ML234 and 06-DA9036I, DFG Cluster of Excellence Origin and Structure of the Universe, the European Community under TMR contract TMR ERBFMRX CT97-0123, HPRI contracts HPRICT-1999-00018 and HPRI-CT-2001-50033, ENSAR and EURONS contract RII3-CT-2004-506065, the BriX IAP research programs P5/07 and P6/23, FWO-Vlaanderen, GOA/2004/03 (BOF-KU Leuven), the Swedish Research Council, the UK EPSRC and STFC, the Marie Curie Intra-European Fellowship of the European Community's $7^{\text {th }}$ Framework Program under contract number PIEFGA-2008-219175, the Marie Curie Action grant of the FP7 under contract IEF-GA-2009-252951 and JSPS Fellows (Grant No. 217368). We would also like to thank ISOLDE for delivering all the beams for these experiments.
Open Access This is an open access article distributed under the terms of the Creative Commons Attribution License (http://creativecommons.org/licenses/by/3.0), which permits unrestricted use, distribution, and reproduction in any medium, provided the original work is properly cited.

\section{References}

1. O. Kester et al., Nucl. Instrum. Methods B 204, 20 (2003).

2. J. Eberth et al., Prog. Part. Nucl. Phys. 46, 389 (2001).

3. P. Van Duppen, K. Riisager, J. Phys. G: Nucl. Part. Phys. 38, 024005 (2011).

4. J. Simpson et al., Z. Phys. A 358, 139 (1997).

5. M.A. Deleplanque, R.M. Diamond (Editors), GAMMASPHERE, A National Gamma-ray Facility, The Proposal, March 1988 (Lawrence Berkeley Lab. Publ., 1988) p. 5202 .

6. Workshop on Physics with a Germanium-Mini-Ball, slide report, MPI für Kernphysik, Heidelberg (May 24-26, 1995).

7. J. Eberth et al., Prog. Part. Nucl. Phys. 38, 29 (1997)

8. C. Gund, Diploma thesis, MPI Heidelberg (1996).

9. C. Gund, Doctoral thesis, University of Heidelberg (2000).

10. J. Eberth et al., Prog. Part. Nucl. Phys. 28, 495 (1992).

11. J. Eberth et al., Nucl. Instrum. Methods A 369, 135 (1996).

12. Digital Gamma Finder (DGF-4C Revision D) www.xia. com/DGF_products.html.

13. F.S. Goulding, D.A. Landis, IEEE Trans. Nucl. Sci. 41, No. 4 (1994).

14. T. Kröll et al., Nucl. Instrum. Methods A 371, 489 (1996) and references therein.

15. L. Palafox-Gámir, Doctoral thesis, Universität Heidelberg (1997).

16. J. Simpson et al., Acta. Phys. Pol. B 36, 1383 (2005).

17. D.W. Weißhaar, Doctoral thesis, University of Cologne (2003).

18. M. Lauer, Doctoral thesis, University of Heidelberg (2004).

19. R. Lutter, O. Schaile et al., IEEE Trans. Nucl. Sci. 47, 280 (2000) http://www.bl.physik.uni-muenchen.de/ marabou/html.

20. H. Essel, N. Kurz et al., http://www-win.gsi.de/daq.

21. R. Brun, F. Rademakers et al., http://root.cern.ch.

22. J. Van de Walle et al., Eur. Phys. J. A 42, 401 (2009).

23. J. Van de Walle et al., Phys. Rev. C 79, 014309 (2009).

24. L.P. Gaffney et al., (Proposal: CERN-INTC-2008-021), submitted to Nature (2013).

25. M. Albers et al., Phys. Rev. Lett. 108, 062701 (2012).

26. N. Kesteloot, MSc thesis, KU Leuven (2010).

27. O. Niedermaier, Doctoral thesis, University of Heidelberg (2008).

28. V. Bildstein et al., Eur. Phys. J. A 48, 85 (2012).

29. J. Allison et al., IEEE Trans. Nucl. Sci. 53, 270 (2006).

30. V. Bildstein et al., Prog. Part. Nucl. Phys. 59, 386 (2007).

31. A.N. Ostrowski et al., Nucl. Instrum. Methods A 480, 448 (2002).

32. T. Davinson et al., Nucl. Instrum. Methods A 288, 245 (1990). 
33. S.L. Thomas et al., Nucl. Instrum. Methods A 288, 212 (1990).

34. P.J. Sellin et al., Nucl. Instrum. Methods A 311, 217 (1992).

35. V785 ADC http://www.caen.it/.

36. http://www . mesytec. com/datasheets/MADC-32.pdf.

37. N. Kesteloot, private communication.

38. http://www . mesytec.com/datasheets/MUX-16.pdf.

39. http://www.mesytec.com/datasheets/MSI-8.pdf.

40. http://www.mesytec.com/datasheets/MPR16.pdf.

41. http://www.mesytec.com/datasheets/STM-16.pdf.

42. J. Diriken et al., submitted to Phys. Rev. Lett. (2013).

43. J. Diriken, private communication.

44. K. Wimmer et al., Phys. Rev. Lett. 105, 252501 (2010).

45. N. Patronis et al., in preparation (Proposal: CERNINTC-2008-007 (2008)).

46. R. Orlandi et al., in preparation (Proposal: CERN-INTC2009-017 (2008)).

47. K. Wimmer et al., in preparation (Proposal: CERNINTC-2009-034).

48. E. Bouquerel et al., Eur. Phys. J. ST 150, 277 (2007).

49. U. Köster et al., Radiochim. Acta 89, 749 (2001).

50. J. Lettry et al., Nucl. Instrum. Methods B 126, 130 (1997).

51. F. Ames et al., Nucl. Instrum. Methods A 538, 17 (2005).

52. A. Gustafsson et al., Nucl. Instrum. Methods A 626, 8 (2011).

53. REX-ISOLDE Collaboration (F. Wenander et al.), CERN-OPEN-2000-320.

54. B. Wolf et al., Nucl. Instrum. Methods B 204, 428 (2003).

55. F. Wenander et al., Nucl. Instrum. Methods B 266, 4346 (2008).

56. G. Pascovici, $40 \mathrm{MHz}$ clock module IKP 5006 (2001).

57. J. Cub et al., Nucl. Instrum. Methods A 453, 522 (2000).

58. P. Bednarczyk et al., Acta. Phys. Pol. B 38, S1293 (2007).

59. W. Weinzierl et al., Diploma thesis, TU Munich (2006).

60. E. Clément et al., private communication (2010).

61. V.N. Fedosseev et al., Nucl. Instrum. Methods B 266, 4378 (2008).

62. P. Reiter et al., in preparation (Proposal: CERN-INTC2010-064 (2010)).

63. I. Stefanescu et al., Phys. Rev. Lett. 100, 112502 (2008).

64. A. Ekström et al., Phys. Rev. C 80, 054302 (2009).

65. J. Diriken et al., Phys. Rev. C 82, 064309 (2010).

66. A. Ekström et al., Eur. Phys. J. A 44, 355 (2010).

67. R. Kirchner, Rev. Sci. Instrum. 67, 928 (1996).

68. ISOLDE on-line yield database, http://oraweb.cern. $\mathrm{ch} / \mathrm{pls} /$ isolde/query_tgt.

69. N. Bree et al., Phys. Rev. C 78, 047301 (2008).

70. A.M. Hurst et al., Phys. Rev. Lett. 98, 072501 (2007).

71. E. Clément et al., in preparation (Proposal: CERNINTC-2006-033 (2006)).

72. P. Delahaye et al., Eur. Phys. J. A 25, 739 (2005).

73. C. Bauer et al., Phys. Rev. C 86, 034310 (2012).

74. J. Van de Walle et al., in preparation.

75. J. Van de Walle et al., Phys. Rev. Lett. 99, 142501 (2007).

76. J.J. He et al., Phys. Rev. C 80, 042801(R) (2009).

77. O. Niedermaier et al., Phys. Rev. Lett. 94, 172501 (2005).

78. V. Bildstein, Doctoral thesis, TU Munich (2010).

79. M. Seidlitz et al., Phys. Lett. B 700, 181 (2011).

80. I. Stefanescu et al., Phys. Rev. Lett. 98, 122701 (2007).

81. E. Rapisarda et al., Phys. Rev. C 84, 064323 (2011).
82. G. Georgiev et al., in preparation (Proposal: CERNINTC-2009-019 (2009)).

83. B.S. Nara Singh et al., in preparation (Proposal: CERNINTC-2008-042).

84. D. Mücher et al., Prog. Part. Nucl. Phys. 59, 361 (2007).

85. D. Mücher, Doctoral thesis, University of Cologne (2009).

86. M. Albers, Doctoral thesis, University of Cologne (2011).

87. T. Behrens, Doctoral thesis, TU Munich (2009).

88. S. Bönig et al., in preparation (Proposal: INTC-2007013).

89. A. Ekström et al., Phys. Rev. Lett. 101, 012502 (2008).

90. D. DiJulio et al., Eur. Phys. J. A 48, 105 (2012).

91. D. DiJulio et al., Phys. Rev. C 86, 031302 (2012).

92. J. Cederkäll et al., Phys. Rev. Lett. 98, 172501 (2007).

93. P. Butler et al., in preparation (Proposal: CERN-INTC2006-034 (2006)).

94. J. Pakarinen et al., in preparation (Proposal: CERNINTC-2008-025 (2008)).

95. B. Bastin et al., in preparation (Proposal: CERN-INTC2008-027 (2008)).

96. A. Robinson et al., in preparation (Proposal: INTC-2007$027(2007))$.

97. C. Thibault et al., Phys. Rev. C 12, 644 (1975).

98. O. Sorlin, M.-G. Porquet, Prog. Part. Nucl. Phys. 61, 602 (2008).

99. F. Marechal et al., Phys. Rev. C 72, 044314 (2005).

100. S. Franchoo et al., Phys. Rev. C 64, 054308 (2001).

101. J. Van Roosbroeck et al., Phys. Rev. C 69, 034313 (2004).

102. S. Lenzi et al., Phys. Rev. C 82, 054301 (2010).

103. A. Görgen et al., J. Phys. G: Nucl. Part. Phys. 37, 103101 (2010).

104. J. Ljungvall et al., Phys. Rev. Lett. 100, 102502 (2008).

105. N. Marginean et al., Phys. Rev. C 80, 021301 (2009).

106. S. Naimi et al., Phys. Rev. Lett. 105, 032502 (2010).

107. C. Sotty et al., in preparation.

108. J.L. Wood, K. Heyde, W. Nazarewicz, M. Huyse, P. Van Duppen, Phys. Rep. 215, 101 (1992).

109. R. Julin, K. Helariutta, M. Muikku, J. Phys. G: Nucl. Part. Phys. 27, R109 (2001).

110. J. Lettry et al., Nucl. Instrum. Methods B 126, 170 (1997).

111. H.J. Wollersheim et al., Nucl. Instrum. Methods A 537, 637 (2005).

112. A. Maj et al., Nucl. Phys. A 571, 185 (1994).

113. P. Doornenbal, Doctoral thesis, Universität zu Köln (2007).

114. R. Lozeva et al., Nucl. Instrum. Methods A 562, 298 (2006).

115. P. Doornenbal et al., Phys. Lett. B 647, 237 (2007).

116. P. Maierbeck et al., Phys. Lett. B 675, 22 (2009).

117. C. Rodriguez-Tajes et al., Acta Phys. Pol. B 40, 859 (2009).

118. M. Facina et al., Nucl. Instrum. Methods Phys. Res. Sect. B 226, 401 (2004).

119. D. Pauwels et al., Nucl. Instrum. Methods Phys. Res. Sect. B 226, 4600 (2008).

120. L. Weissman et al., Nucl. Instrum. Methods A 423, 328 (1999).

121. D. Pauwels et al., Phys. Rev. C 79, 044309 (2009).

122. D. Pauwels et al., Phys. Rev. C 78, 041307(R) (2008).

123. R. Ferrer et al., Phys. Rev. C 81, 044318 (2010).

124. D. Pauwels et al., Phys. Rev. C 86, 064318 (2012). 